Louisiana State University

LSU Digital Commons

1995

\title{
The Interview for the Diagnosis of Eating Disorders-Iv: Application to DSM-IV Diagnostic Criteria.
}

Vesna Kutlesic

Louisiana State University and Agricultural \& Mechanical College

Follow this and additional works at: https://digitalcommons.Isu.edu/gradschool_disstheses

\section{Recommended Citation}

Kutlesic, Vesna, "The Interview for the Diagnosis of Eating Disorders-Iv: Application to DSM-IV Diagnostic Criteria." (1995). LSU Historical Dissertations and Theses. 6027.

https://digitalcommons.Isu.edu/gradschool_disstheses/6027

This Dissertation is brought to you for free and open access by the Graduate School at LSU Digital Commons. It has been accepted for inclusion in LSU Historical Dissertations and Theses by an authorized administrator of LSU Digital Commons. For more information, please contact gradetd@lsu.edu. 


\section{INFORMATION TO USERS}

This manuscript has been reproduced from the microfilm master. UMI films the text directly from the original or copy submitted. Thus, some thesis and dissertation copies are in typewriter face, while others may be from any type of computer printer.

The quality of this reproduction is dependent upon the quality of the copy submitted. Broken or indistinct print, colored or poor quality illustrations and photographs, print bleedthrough, substandard margins, and improper alignment can adversely affect reproduction.

In the unlikely event that the author did not send UMI a complete manuscript and there are missing pages, these will be noted. Also, if unauthorized copyright material had to be removed, a note will indicate the deletion.

Oversize materials (e.g., maps, drawings, charts) are reproduced by sectioning the original, beginning at the upper left-hand corner and continuing from left to right in equal sections with small overlaps. Each original is also photographed in one exposure and is included in reduced form at the back of the book.

Photographs included in the original manuscript have been reproduced xerographically in this copy. Higher quality $6^{\prime \prime} \times 9^{n}$ black and white photographic prints are available for any photographs or illustrations appearing in this copy for an additional charge. Contact UMI directly to order.

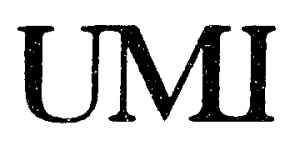

A Bell \& Howell Information Company 


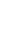


THE INTERVIEW FOR THE DIAGNOSIS OF EATING DISORDERS-IV: APPLICATION TO DSM-IV DIAGNOSTIC CRITERIA

\section{A Dissertation}

Submitted to the Graduate Faculty of the Louisiana State University and Agricultural and Mechanical College in partial fulfillment of the requirements for the degree of Doctor of Philosophy

in

The Department of Psychology

Vesna Kutlesic

B.A. Kent state University, 1988

M.A., Louisiana State University, 1992 August 1995 
UMI Number: 9609101

UMI Microform 9609101

Copyright 1996, by UMI Company. All rights reserved.

This microform edition is protected against unauthorized copying under Title 17, United States Code.

\section{UMI}

300 North Zeeb Road

Ann Arbor, MI 48103 


\section{Acknowledgements}

Upon completion of these many years of study, I find myself indebted to many individuals who have provided the guidance and encouragement that were essential toward the completion of my graduate career. First and foremost, I am indebted to my dissertation chairman, Dr. Donald Williamson, who has been both a pleasure to work with and a role model with regard to exemplary work both as a researcher and clinician. I am also grateful to my dissertation committee members, Drs. Claire Advokat, Robert Roy Allen, David Blouin, Mary Lou Kelley, and Jack Parker for their input and guidance toward the completion of this dissertation project.

I am thankful to the individuals who agreed to participate in this study at Louisiana state University, Our Lady of the Lake Regional Medical Center, and the Renfrew Center in Pennsylvania. I am extremely grateful to my fellow graduate students and coworkers who agreed to serve as interviewers in the current study. These individuals include Jane Barbin, Jodie Rabalais, Bret Bentz, Drew Anderson, Lori Jackman, Kathleen Murphy Aberenz, Dr. Erich Duchmann, Dr. C. J. Davis, and Dr. David Gleaves. Special thanks to Jane Barbin for all her assistance with my data collection. In addition, I am grateful to Colleen Brown and Cyndi Moore for all of their organizational and clerical support. 
I have been blessed with many wonderful friends who have been invaluable to me throughout my graduate career, and in my life before my graduate career. For fear that I may not mention someone who deserves to be mentioned, I will only give special recognition to Dr. Staci Guidry and Dr. Katherine wickstrom who have both professionally and personally been godsends, and who are responsible for many of the pleasant memories I will have of my graduate school experience. And finally, I would like to thank my family, particularly my parents, Ostoja and Slavka Kutlesic. They have sacrificed much of themselves to provide me with the foundation for a good life, and I hope that I will be able to emulate the integrity, generosity, and dedication that they have modeled for me. 


\section{Table of contents}

Acknowledgements . . . . . . . . . . . . . . . . ii

List of Tables . . . . . . . . . . . . . . . vi

List of Figures . . . . . . . . . . . . . . viii

Abstract . . . . . . . . . . . . . . . . ix

Introduction . . . . . . . . . . . . . . . . . 1

Types of Interviews . . . . . . . . . . . . . . . 4

Historical Evolution of the Structured Interview. .5

Diagnostic Criteria that Preceded the Major

Structured Interviews . . . . . . . . . . 10

Construction of the Modern Diagnostic Interviews. 23

Development of Interview Schedules for specific

Diagnoses . . . . . . . . . . . . 29

Strategy for the Development of the IDED-IV . . . . 37

Phase I: Development of the IDED-IV . . . . . . 37

Changes in structure from an Earlier

Version of the IDED . . . . . . . . . 37

Format and Content of the IDED-IV . . . . . 38

Phase II: Reliability of the IDED-IV. . . . . . 40

Phase III: Validity of the IDED-IV . . . . . . . 41

Method . . . . . . . . . . . . . . . . 52

Subjects . . . . . . . . . . . . . . . . 52

Initial Sample . . . . . . . . . . . 52

Validation Sample. . . . . . . . . . . 62

Assessment Measures . . . . . . . . . 67

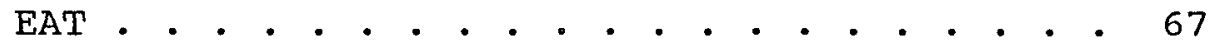

BULIT-R . . . . . . . . . . . . . . 68

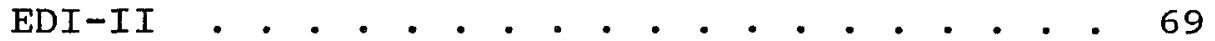

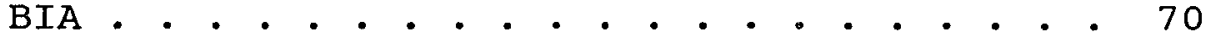

Procedure . . . . . . . . . . . . . . . . . . 71

Phase II: Reliability study... . . . . . 71

Phase III: Validity Study . . . . . . . . 77

Results . . • . . . . . . . . . . . . . . . 79

Phase II: Reliability of the IDED-IV . . . . . 79

Internal Consistency . . . . . . . . . . 79

Interrater Reliability . . . . . . . . 82

Phase III: Validity of the IDED-IV . . . . . . 89

Convergent and Discriminant Validity . . . 89 


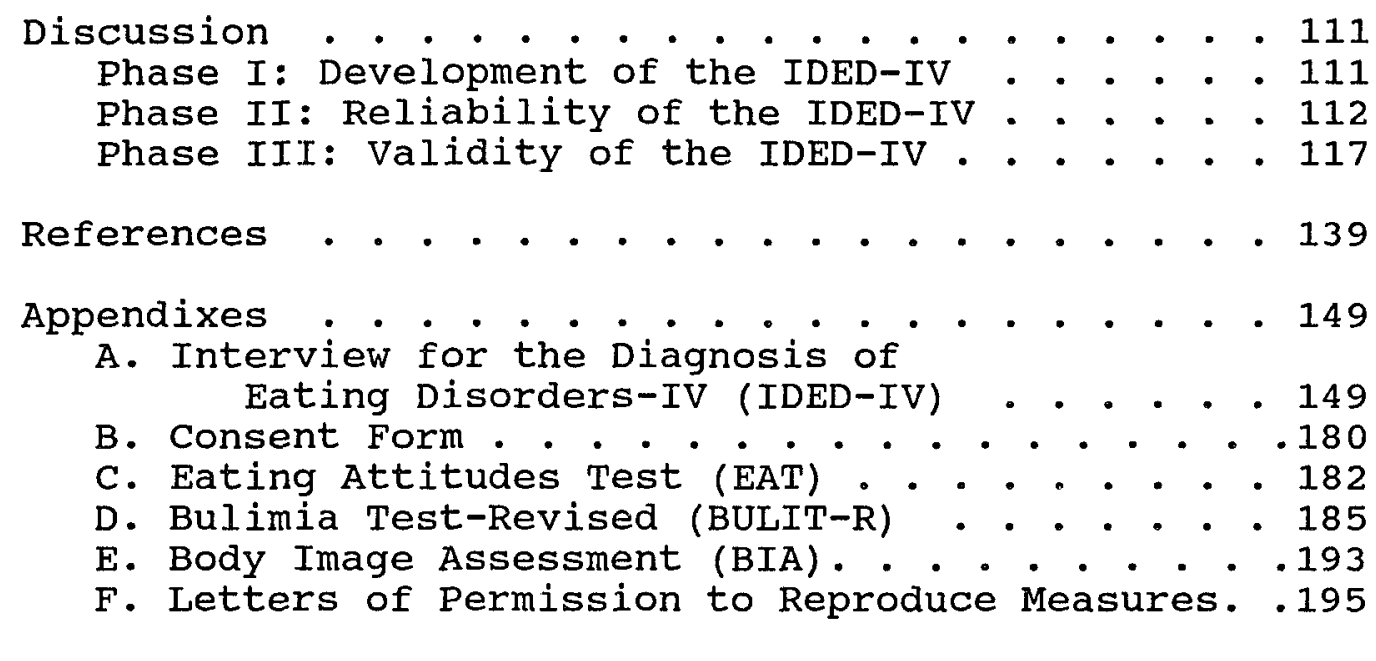

vita . . . . . . . . . . . . . . 198 


\section{List of Tables}

1. DSM-III-R and DSM-IV Criteria for

Anorexia Nervosa. . . . . . . . . . . 16

2. DSM-III-R and DSM-IV Criteria for

Bulimia Nervosa . . . . . . . . . . . 18

3. DSM-III-R and DSM-IV Criteria for Eating

Disorder Not Otherwise Specified . . . . . 20

4. DSM-IV Criteria for Binge-Eating Disorder . . . 22

5. Predicted Intercorrelations Between the IDED-IV Subscale Totals . . . . . . . . . 43

6. Predicted Intercorrelations Between the IDED-IV Subscale Totals and Scores on Self-Report Measures of Eating Disorder Symptoms . . . . . . . . . . . . . . . 44

7. Descriptive variables for clinical and nonclinical subjects in the initial sample . . . 53

8. Eating Disorder Diagnoses in the Clinical Group for the Initial Pool of Subjects . . . 61

9. Descriptive Variables for clinical Subjects in the Validation Sample . . . . . 66

10. Item-total Correlations Between IDED-IV Individual Ratings and Subscale Totals . . . 80

11. Percent Agreement for Ratings Between Interviewers to Indicate Presence or Absence of Eating Disorder Symptoms . . . . 84

12. Comparison of Spearman Rank-Order Correlation Coefficients and Percent Agreement for IDED-IV Ratings Made by Two Independent Raters . . . . . . . . . .

13. Intercorrelations Between the IDED-IV Subscale Totals . . . . . . . . . . 91

14. Intercorrelations Between the IDED-IV Subscale Totals and Scores on Self-Report Measures of Eating Disorder Symptoms 
15. Significant Canonical Correlations Between Predictor IDED-IV subscale Totals and Criterion Dependent Measures . . . . . . 98

16. Analysis of Variance and Scheffe Post-Hoc Comparisons of Group Means for Dependent Measures . . . . . . . . . . 105 


\section{List of Figures}

1. Decision Tree for Making DSM-IV Anorexia Nervosa Diagnosis and its Subclinical

Variations Subsumed under Eating Disorder Not Otherwise Specified (ED NOS) . • . • 56

2. Decision Tree for Making DSM-IV Binge-Eating Disorder Diagnosis and its Subclinical Variations subsumed under Eating Disorder Not otherwise Specified (ED NOS) . . . . 57

3. Decision Tree for Making DSM-IV Bulimia Nervosa Diagnosis with Subclinical Variations Subsumed under Eating Disorder Not Otherwise Specified (ED NOS). •. . . . . . . . . . . . 58 
Abstract

The standard diagnostic system in the United states, the Diagnostic and Statistical Manual III-Revised, has recently been revised (i.e., DSM-IV) to include recent research and clinical advances that have enabled further specification of the existing diagnostic criteria. One set of diagnostic criteria that is representative of this increased specification are the criteria for the eating disorders. The expansion of these criteria in the recent publication of DSM-IV creates a need for the development of a structured interview that would enable differential diagnosis among these diagnostic categories. Thus, the purpose of the current study was to develop and evaluate the psychometric properties of a semi-structured diagnostic interview for DSM-IV eating disorder diagnoses. The Interview for the Diagnosis of Eating Disorders-IV (IDEDIV) was developed for this purpose. The study was conducted in three phases. Phase I involved the development of the IDED-IV which involved the application of DSM-IV criteria and changes made to the content and format of an earlier version of the IDED (i.e., IDED-III). Phase II involved examination of the reliability of the IDED-IV. High levels of internal consistency and interrater reliability for diagnosis were found. Adequate to high interrater reliability was found for the individual ratings within the IDED-IV Subscales. Phase III focused on 
establishing the convergent and discriminant validity of the IDED-IV. The IDED-IV subscales were found to be related to each other and to self-report measures of eating disorder symptoms. In addition, significant differences between clinical and control groups identified by the IDEDIV were also found on measures of related and unrelated constructs. Thus, the convergent and discriminant validity of the IDED-IV was supported. 


\section{Introduction}

Classification is basic to science (Wiens, 1990). Hypothesis formation and testing in science depends on the availability of accurate and reliable descriptions of phenomena that permit differentiation and prediction (Wiens \& Matarazzo, 1983). In a similar manner, diagnosis in clinical practice introduces order into the clinician's observations, increases their meaningfulness, and may ultimately lead to prevention and amelioration of the clinical disorder. By placing an object or organism or set of behaviors into a certain class, the inference of certain characteristics, and the consideration of individual characteristics within a broader context, becomes possible.

The clinical interview has been devised in order to obtain information necessary for arriving at diagnostic formulations for both research and clinical purposes (Wiens, 1990), and has been identified as a key tool for research in mental health (Shea, 1990). Researchers have used clinical interviews to obtain careful diagnostic delineation of subjects' symptoms and characteristics. Such information enables the study of homogeneous groups of subjects, and the definition of groups of subjects who are comparable to subjects being studied by researchers in other settings (Wiens, 1990). Clinicians have relied on clinical interviews to obtain information for arriving at diagnoses to aid in treatment planning (Wiens, 1990). 
With the recent advances in the treatments available for specific clinical disorders, determination of patients' diagnoses is a critical step toward matching patients to appropriate treatment modalities.

As the knowledge base for the majority of clinical disorders has expanded, and the corresponding diagnostic criteria have become increasingly more detailed, the need for specialized assessment instruments focused on specific clinical disorders has become apparent. The standard diagnostic system in the United states, the Diagnostic and Statistical Manual III-Revised (DSM-III-R: American Psychiatric Association, 1987), has recently been revised (i.e., DSM-IV) to include recent research and clinical advances that have enabled further specification of the existing diagnostic criteria. One set of diagnostic criteria that is representative of this increased specification are the criteria for the eating disorders. The expansion of these criteria in DSM-IV create the need for the development of a structured clinical interview that enables differential diagnosis among these diagnostic categories. A structured interview would enable both researchers and clinicians to arrive at diagnostic decisions in a timely manner, to reduce the cost and risk that subjects would prematurely discontinue participation 
during an assessment. A structured clinical interview for the eating disorders which is based upon DSM-IV criteria has not been developed.

The complexity of the attitudinal and behavioral features that are specific to eating disorder psychopathology necessitate the development of a structured, clinical interview that enables the assessment of these dimensions (Cooper \& Fairburn, 1987). A structured clinical interview allows the detailed probing and questioning of subjects to determine whether reported symptoms represent pathologic body image and eating attitudes and behaviors, or symptoms which are subjectively distressing, but widely held in our weight conscious society (Rosen, Vara, Wendt, \& Leitenberg, 1990). In addition, structured interviews include objective definitions for behavioral symptoms (e.g., binge-eating, purging, dieting) to improve the likelihood of diagnostic agreement across interviewers. Finally, a clinical interview would be useful for calculating the frequencies and time frame of specific behaviors that are relevant to diagnostic decisions, or for the assessment of treatment outcome.

The purpose of the current study is to develop a semistructured diagnostic interview that provides diagnoses based on the recently published DSM-IV eating disorder diagnostic criteria. Before describing the proposed 
development of this semi-structured interview, the basis for the current interview will be provided by reviewing: a) the types of interviews that have been developed, b) the historical evolution of the structured clinical interview, and c) the psychometric development of several commonly used structured clinical interviews. Types of Interviews

The different types of clinical interviews can be differentiated on two primary dimensions: standardization and scheduling (Shea, 1990; Richardson, Dohrenwend, \& Klein, 1965). Standardization refers to the extent to which areas of information to be explored are specified in the interview procedure. Scheduling refers to the prespecification of the wording and sequence of the interview process. The amount of emphasis on either or both of these dimensions determines the level of structure within a clinical interview. There are four general types of interviews that are characterized by different degrees of structure.

First, free format interviews have a minimal amount of standardization and scheduling, and consequently are the least structured of the different interview types. The focus of free format interviews on the spontaneous statements made by individuals involves minimal standardization, and the practice of posing interview questions based on the patient's lead requires minimal 
scheduling within the interview. In contrast, fully structured interviews contain the most structure, and involve the highest degree of standardization and scheduling. Fully structured interviews are highly standardized in that detailed informational areas must be covered, and highly scheduled in that assessment information must be obtained in a specified manner. The semi-structured and flexibly structured interviews represent intermediate levels of structure. Semi-structured interviews are standardized with regard to the content to be explored, but the scheduling is only moderately predetermined. General guidelines may be provided, but the interviewer is given some latitude to move within this framework. In a similar vein, flexibly structured interviews depend on a standardized database, but have no limits on scheduling. The interviewer is likely to begin with topics that appear to be the most pressing for an interviewee, and then add structure gradually after the interviewee is engaged in the interview. Thus, the actual scheduling will be unique to each interviewer-interviewee dyad. Historical Evolution of the Structured Interview

The historical evolution of the clinical interview has paralleled the development of the theoretical knowledge base that prevailed during a given age (Shea, 1990). In particular, the more numerous and syndrome-specific the 
available treatment modalities were, the more likely that a standardized database with clearly delineated diagnostic criteria would be developed. Moreover, once a particular standardized database became large, a gradual shift toward methods of structuring, either by rigid scheduling or flexible maneuvering, would occur to make the gathering of data more efficient and manageable.

In the early 1900 's, the first attempts were made toward the classification of mental illness, with a focus on the careful detailing of behaviors and symptoms, and the goal of determining specific syndromes and diseases (Kaplan, Freedman, \& Sadock, 1980). During this time, a psychiatrist by the name of Adolf Meyer proved to be a catalyst in the development of the psychiatric interview (Shea, 1990; Meyer, 1951). Meyer proposed a psychobiological approach to assessment, which entailed obtaining a biography of the biological, historical, psychological, and social influences on an individual's current behavior (Kaplan et al., 1980; Meyer, 1951). Meyer's focus on psychological and social influences promoted the development of a free format style of interviewing, while his focus on biological influences and current symptomatology promoted the semi-structured and flexibly-structured interview formats. 
By the mid $1920^{\prime} \mathrm{s}$, many of the major components of the psychiatric interview were established (Shea, 1990). The key content areas included the chief complaint, the history of present illness, social history, family history, medical history, and mental status. Specific diagnoses were not the focus of these early interviews, due to the absence of a diagnostic system with well-delineated diagnostic criteria. Thus, clinical interviews during this period were not based on a standardized database. In addition, with the paucity of diagnosis-specific treatment modalities available, there did not exist a need for scheduling to obtain specific information in a timely manner. As a result, the free format interview style flourished, with the purpose of obtaining a general diagnostic overview.

By 1940, psychoanalysis became well established in America (Shea, 1990). Sigmund Freud's pioneering work (1910, 1927) had an enormous impact on interviewing technique. His basic theories moved away from the emphasis on diagnosis in a medical sense, toward a more probing investigation of actual psychological processes. With the development of ego psychology and the investigation of defense mechanisms by theorists such as Heinz Hartman (1958) and Anna Freud (1946), the emphasis further shifted toward an understanding of how the individual's defenses 
were manifested in the context of the interview itself. Interviewing and therapy seemed to become less distinct, and a free format style of interviewing prevailed.

In the early 1950's, Sullivan (1954) stressed the importance of viewing the interview as a sociological phenomenon in which the patient and clinician formed a unique and dynamic dyad, with the behavior of each affecting the other. One of Sullivan's key concepts was participant observation. This concept emphasized the need of the interviewer to "step aside" during the interview and to evaluate his or her own behavior and the impact of that behavior on the patient. He was also one of the first interviewers to emphasize the importance of scheduling, and discussed specific methods of making transitions during the interview from one topic to another. Sullivan's recognition of the importance of both the free format style and scheduling issues, led to the development of a flexibly structured style of interviewing in which these various techniques could be utilized at the discretion of the interviewer.

Since the early 1950 's, several revolutionary changes in the conceptualization and treatment of psychopathology have promoted the development of structured interviews that contain a high degree of standardization and scheduling (Groth-Marnat, 1990; Shea, 1990; Blain \& Barton, 1979). Revisions in the major diagnostic systems towards more 
descriptive and symptom-based diagnoses, combined with the proliferation of diagnosis-specific treatment interventions, have promoted the development of structured interviews that contain a high degree of standardization (Groth-Marnat, 1990; Shea, 1990; Blain \& Barton, 1979). Moreover, the reduced length of hospital stay necessitated that assessment information be collected in an efficient manner, which led to the increased scheduling of clinical interviews. In addition, empirical studies required that homogeneous groups of subjects be identified so that results across studies could be compared and contrasted (Rubinson \& Asnis, 1989). Thus, structured interviews became widely utilized in an effort to improve the rate of agreement across interviewers, and to reduce cost through use of lay interviewers.

An additional incentive for the development of structured clinical interviews was the observation that free format style interviews had poor reliability across interviewers (Groth-Marnat, 1990; Wiens, 1990; Ash, 1949). The two primary factors leading to the poor reliability of these early interviews were identified as being criterion and information variance (Spitzer, Endicott, \& Robins, 1975). Criterion variance refers to differences in the inclusion and exclusion criteria that interviewers use to summarize patient data into psychiatric diagnoses. Early classification systems provided brief, general descriptions 
of diagnostic categories that consisted of a list of common features of each disorder, but did not clearly specify which signs and symptoms were required for a particular diagnosis. Therefore, interviewers made diagnoses based on variable sets of assessment information, which reduced the level of interrater reliability.

A second factor that contributed to the poor reliability of free format interviews was information variance. Information variance refers to variability in the amount and type of information derived by interviewers during interviews with patients. Interviewers are less likely to agree on the diagnosis for a particular patient when they have disparate interviewing styles, vary the topics covered, and/or rely on different sources of assessment information (e.g., patient's self-report, interview with significant other). In an effort to control for information variance, structured clinical interviews were developed. Structured clinical interviews reduce information variance by specifying the information to be obtained from each interviewee.

\section{Diagnostic Criteria that Preceded the Major structured} Interviews

The evolution of the most commonly used structured interviews corresponded to developments in the diagnostic system available during a given age (Rubinson \& Asnis, 1989). The diagnostic systems that have been in existence 
have emphasized a descriptive, syndromal rather than a theoretical approach. The descriptive approach attempts to bypass controversies among clinicians having different theoretical orientations regarding the cause of many clinical disorders. Empirical studies of the diagnostic reliability of these classification systems have been conducted to determine whether the diagnostic discriminations made with these systems are consistent and replicable. These studies found support for improved reliability and reduced criterion variance in the diagnostic decisions made by researchers and clinicians who referred to a standard diagnostic system. Consequently, the refinement of these diagnostic systems, and the development of structured interviews aimed at reducing information variance became important areas of research.

The first diagnostic system that included specific criterion-based diagnoses was developed by Feighner et al. (1972). The Feighner Criteria provided clear, behaviorallyoriented descriptions of 16 psychiatric disorders based on the Diagnostic and Statistical Manual-II (DSM-II: APA, 1968). The criteria were based primarily on the results of empirical studies that examined the characteristics of patients diagnosed with one of the clinical disorders represented in the criteria. The improved diagnostic reliability that resulted when interviewers used the criteria was evident in the high rates of agreement found 
between interviewers ( $86 \%$ to $95 \%$ ). The Feighner Criteria were subsequently incorporated into a structured interview, the Renard Diagnostic Interview (RDI: Helzer, Robins, Croughan, \& Weiner, 1981), in order to facilitate the efficient gathering of information necessary for making diagnostic decisions. The development of the RDI and subsequent interviews will be described in a later section.

The Feighner Criteria were also expanded and modified by Spitzer, Endicott, and Robins (1978), and named the Research Diagnostic Criteria (RDC). The RDC included a glossary of psychiatric definitions plus criteria for 24 diagnostic categories. The major purpose of the RDC was to enable investigators to select relatively homogeneous groups of subjects who met specified diagnostic criteria. Criteria were developed based on research evidence indicating that the criteria chosen had considerable usefulness for such diagnostic purposes as predicting treatment outcome, response to treatment, and familial association. The RDC also included criteria that were important considerations clinically, which had not necessarily been identified empirically. The results of three separate interrater reliability studies found the RDC diagnostic categories highly reliable, with values ranging from .65 to .98 (Spitzer et al., 1978). 
The RDC were initially spurred by the exigencies of research (Katz, Secunda, \& Hirschenfield, 1979); however, they have also had a significant impact on clinical practice. Many of the categories, concepts, and principles of the RDC were incorporated into the Diagnostic and Statistical Manual-III (DSM-III: American Psychiatric Association, 1980). Earlier editions (DSM-I: American Psychiatric Association, 1952; DSM-II: American Psychiatric Association, 1968) provided brief, general descriptions of common features for each disorder, that were based on controversial theoretical notions, and had inadequate reliability and validity (Begelman, 1976). The DSM-III diagnostic criteria were developed to address the problems of the earlier diagnostic systems by providing specific operational criteria whose reliability was supported in several field trials. The results of the DSM-III field trials found support for a high level of interrater reliability for the major classes of Axis I disorders with mean kappa coefficients of .78 for joint interviews, and .66 for diagnoses made after independent interviews by pairs of interviewers (Hyler, williams, \& Spitzer, 1982; Spitzer, Forman, \& Nee, 1979; Williams \& Spitzer, 1980). Nevertheless, the DSM-III diagnostic criteria were later revised, (DSM-III-Revised(R): American Psychiatric Association, 1987), to include changes in the symptoms, duration, and exclusion criteria for some disorders, and 
the ability to report dual diagnoses for diagnostic categories that were previously mutually exclusive. The results of the DSM-III-R field trials provided support for the improved reliability and concurrent and descriptive validity of the revised criteria (i.e., with clinician's diagnoses as the criterion). Field trials were conducted with draft criteria for autism, disruptive behavior disorders, agoraphobia, self-defeating personality disorders, and substance dependence (American Psychiatric Association, 1987; Rounsaville, Kosten, Williams, \& Spitzer, 1987; Spitzer \& Williams, 1988; Spitzer, Williams, Kass, \& Davies, 1989).

More recently, a fourth revision of the Diagnostic and Statistical Manual has been developed (DSM-IV: American Psychiatric Association, 1993). The main innovation of DSMIV is its reliance on a systematic review of the literature as the primary basis for the modification of DSM-III-R diagnostic criteria (First, Frances, Widiger, Pincus, \& Wakefield-Davis, 1992; Widiger, Frances, Pincus, \& Davis, 1991). For DSM-III-R diagnostic categories which did not have recent empirical data available to be reviewed, field trials and data reanalyses of former studies were conducted to guide the modification of these categories in DSM-IV (First et al., 1992).

Among the diagnostic criteria most substantially revised in DSM-IV, are the diagnostic criteria for the 
eating disorders. Tables 1 through 4 detail the specific changes made to the Anorexia Nervosa, Bulimia Nervosa, Eating Disorder Not otherwise Specified, and Binge-Eating Disorder diagnostic criteria. The sections that have been revised or deleted from DSM-III-R are underlined in Tables 1 through 3 in the DSM-III-R column. The recently revised and developed sections that have been added to DSM-IV are both underlined and enclosed in square brackets in Tables 1 through 3 in the DSM-IV column. Binge-Eating Disorder, described in Table 4, was not included in DSM-III-R and therefore is only found in DSM-IV.

The primary changes made to the Anorexia Nervosa diagnostic category in DSM-IV include clarification of the wording for Criteria $A, C$, and $D$, and the addition of the Restricting and Binge-Eating/Purging subtypes (see Table 1). The Bulimia Nervosa criteria were more extensively revised in DSM-IV (see Table 2). Criteria A and B in DSMIII-R were combined to form Criterion $A$ in DSM-IV. In addition, the definitions for binge eating and a lack of control over eating were elaborated upon in DSM-IV. Criteria C, D, and E in DSM-III-R were reworded and labeled Criteria B, C, and D in DSM-IV, respectively. Moreover, in DSM-IV, Criterion E was added which specifies that for a diagnosis of Bulimia Nervosa to be made, the "disturbance 


\section{DSM-III-R}

A. Refusal to maintain body weight over a minimal normal weight for age and height, e.g., weight loss leading to maintenance of body weight $15 \%$ below that expected; or failure to make expected weight gain during period of growth, leading to body weight 15\% below that expected.

B. Intense fear of gaining weight or becoming fat, even though underweight.

C. Disturbance in the way in which one's body weight, size, or shape is experienced, e.g.. the person claims to "feel fat" even when emaciated, believes that one area of the body is "too fat" even when obviously underweight]

D. In females, absence of at least three consecutive menstrual cycles when otherwise expected to occur (primary or secondary amenorrhea). (A woman is considered to have amenorrhea if her periods occur only following hormone, e.g., estrogen administration.)

No equivalent
DSM-IV

A. Refusal to maintain body weight [at or above] a minimally normal weight for age and height (e.g., weight loss leading to maintenance of body weight [less than $85 \%$ of] that expected; or failure to make expected weight gain during period of growth, leading to body weight [less than $85 \%$ of] that expected.

B. Intense fear of gaining weight or becoming fat, even though underweight.

C. Disturbance in the way in which one's body weight or shape is experienced; [undue influence of body weight or shape on self-evaluation, or denial of the seriousness of the current low body weight.]

D. In [post-menarchal]

females, [amenorrhea], i.e., the absence of at least

three consecutive menstrual cycles. (A woman is considered to have amenorrhea if her periods occur only following hormone, e.g., estrogen, administration.)

[Specify type: ] [Restricting Type: During the episode of Anorexia Nervosa, the person does not engage in binge eating or purging behavior (i.e., self-induced vomiting or the misuse of laxatives or diuretics).] 
DSM-III-R
DSM-IV

[Binge Eating/Purging Type: During the episode of Anorexia Nervosa, the person regularly engages in binge eating or purging behavior (i.e., selfinduced vomiting or the misuse of laxatives or diuretics).]

Note. Data in column 1 are from the Diagnostic and Statistical Manual (3rd ed.) (p. 67), by the American Psychiatric Association, 1987, Washington, D. C.: American Psychiatric Association. Data in Column 2 are from the Diagnostic and statistical Manual (4th ed.) (p. 544-545), by the American Psychiatric Association, 1993, Washington, D. C.: American Psychiatric Association. Underlined text in DSM-III-R column has been deleted from DSM-IV, and replaced with underlined text enclosed in square brackets in DSM-IV column. 
DSM-III-R

A. Recurrent episodes of binge eating (rapid consumption of a large amount of food in a discrete period of time).

B. A feeling of lack of control over eating behavior during the eating binges.

c. The person regularly engages in either self-induced vomiting, use of laxatives or diuretics, strict dieting or fasting, or vigorous exercise in order to prevent weight gain.

D. A minimum average of two binge eating episodes a week for at least three months.

E. Persistent overconcern with body shape and weight.

\section{$\underline{D S M-I V}$}

A. Recurrent episodes of binge eating. [An episode of binge eating is characterized both of the following: ]

[1) eating, in a discrete period of time (e.q., within any two hour period, an amount of food that is definitely larger than most people would eat during a similar period of time and under similar circumstances, and, ]

[2] a sense of lack of control over eating during the episode (e.g., a feeling that one cannot stop eating or control what or how much one is eating).]

B. [Recurrent inappropriate compensatory behavior in order to provent weight gain, such as: seif-induced vomiting, misuse of laxatives. diuretics or other medications; fasting; or excessive exercise.]

c. The binge eating and inappropriate compensatory behaviors both occur, on average, at least twice a week for three months.]

D. [Self-evaluation is unduly influenced by body shape and weight.]

E. [The disturbance does not occur exclusively during episodes of Anorexia Nervosa] 
DSM-III-R

No equivalent
DSM-IV

[Specify Type] :

[Purging Type: the person regularly engages in selfinduced vomiting or the misuse of laxatives or diuretics.]

[Nonpurging Type: the person uses other inappropriate compensatory behaviors. such as fasting or excessive exercise, but does not regularly engage in selfinduced vomiting or the misuse of laxatives or diuretics.]

Note. Data in column 1 are from the Diagnostic and Statistical Manual (3rd ed.) (p. 549-550), by the American Psychiatric Association, 1987, Washington, D. C.: American Psychiatric Association. Data in Column 2 are from the Diagnostic and Statistical Manual (4th ed.) (p. 68-69), by the American Psychiatric Association, 1993, Washington, D. C.: American Psychiatric Association. Underlined text in DSM-III-R column has been deleted from DSM-IV, and replaced with underlined text enclosed in square brackets in DSM-IV column. 
DSM-III-R and DSM-IV Criteria for Eating Disorder Not otherwise specified

DSM-III-R

Disorders of eating that do not meet the criteria of a specific Eating Disorder. Examples:

1. all of the features of Anorexia Nervosa in a female except absence of menses.

2. no equivalent
$\underline{D S M-I V}$

[This category is for] disorders of eating that do not meet the criteria for any specific Eating Disorder. Examples [include] :

1. all of the [criteria] for Anorexia Nervosa [are met except the individual has regular menses.]

[2. all of the criteria for Anorexia Nervosa are met except that, despite significant weight loss, the individual's current weight is in the normal range.]

3. all of the [criteria for] Bulimia Nervosa lare met except binges occur at a frequency of less than twice a week or for a duration of less than three months.]

4. a person of average weight who does not have binge eating episodes, but frequently engages in self-induced vomiting for fear of gaining weight.

5. no equivalent
4. [an individual of normal body weight who regularly engages in inappropriate compensatory behavior after eating small amounts of food (e.g., self-induced vomiting after the consumption of two cookies)]

[5. an individual who repeatedly chews and spits out, but does not swallow, large amounts of food.] 
$\underline{D S M-I I I-R}$

6. no equivalent $\underline{\text { DSM-IV }}$

[6. Binge eating disorder: recurrent episodes of binge eating in the absence of the inappropriate compensatory behaviors characteristic of Bulimia Nervosa] (see Table 4 for detailed criteria).

Note. Data in column 1 are from the Diagnostic and Statistical Manual (3rd ed.) ( $p .71)$, by the American Psychiatric Association, 1987, Washington, D. C.: American Psychiatric Association. Data in Column 2 are from the Diagnostic and Statistical Manual (4th ed.) (p. 550), by the American Psychiatric Association, 1993, Washington, D. C.: American Psychiatric Association. Underlined text in DSM-III-R column has been deleted from DSM-IV, and replaced with underlined text enclosed in brackets in DSM-IV column. 
Table 4

DSM-IV Criteria for Binge Eating Disorder

A. Recurrent episodes of binge eating. An episode of binge eating is characterized by both of the following:

(1) eating in a discrete period of time (e.g., within any two hour period), an amount of food that is definitely larger than most people would eat during a similar period of time under similar circumstances

(2) a sense of lack of control over eating during the episode (e.g., a feeling that one cannot stop eating or control what or how much one is eating).

B. The binge eating episodes are associated with at least three of the following:

(1) eating much more rapidly than normal

(2) eating until feeling uncomfortably full

(3) eating large amounts of food when not feeling physically hungry

(4) eating alone because of being embarrassed by how much one is eating

(5) feeling disgusted with oneself, depressed or feeling very guilty after overeating

c. Marked distress regarding binge eating.

D. The binge eating occurs, on average, at least two days a week for six months.

E. The binge eating is not associated with the use of inappropriate compensatory behaviors (e.g., purging, fasting, excessive exercise) and does not occur exclusively during the course of Anorexia Nervosa or Bulimia Nervosa.

Note. From the Diagnostic and statistical Manual (4th ed.) (p. 731), by the American Psychiatric Association, 1993, Washington, D. C.: American Psychiatric Association. 
does not occur exclusively during episodes of Anorexia Nervosa." Finally, the Purging and Nonpurging Types were added to the DSM-IV Bulimia Nervosa diagnosis.

The diagnostic criteria for the Eating Disorder Not Otherwise Specified (ED NOS) diagnosis in DSM-III-R has also been modified in DSM-IV (see Table 3). The wording for the three aberrant eating patterns described in DSM-III-R was revised in DSM-IV. Three new atypical eating patterns were also added to the ED NOS diagnosis in DSM-IV for which there are no equivalent diagnoses in DSM-III-R, as indicated in Table 3. Among these, the Binge-Eating Disorder diagnostic category has been added to DSM-IV (see Table 4).

Construction of the Modern Diagnostic Interviews

The establishment of standard diagnostic criteria necessitated the development of structured interviews that would promote the collection of data in a timely manner and aid in differential diagnosis (Wiens, 1990). The majority of these interviews incorporated the criteria of the diagnostic system that prevailed during the time of the development of the interview. The focus of early studies which described the development of the major structured interviews was on establishing reliability due to the recognition that criterion and information variance were the primary sources of interrater disagreement (Spitzer \& Fleiss, 1974). Validity and additional reliability studies 
of the existing interviews followed in the years after the initial publication of the interviews.

The Renard Diagnostic Interview (RDI: Helzer et al., 1981) was developed to aid in the collection of information needed to arrive at diagnoses based on the Feighner Criteria. In addition to questions related to symptoms, the RDI covers demographic information, history of physical illness, family history of psychiatric disorder, age at onset of each disorder, and a mental status examination. Interrater reliability was evaluated in a study of 120 psychiatric patients, who were first interviewed by a psychiatrist using a standard research interview, and then administered the RDI by either two psychiatrists $(n=36)$, two lay interviewers $(n=36)$, or one of each $(n=48)$. The raters included 10 psychiatrists and four lay interviewers. Mean kappa values across diagnostic categories were indicative of moderate levels of agreement between pairs of psychiatrists (kappa $=.52)$, pairs of lay-interviewers (kappa $=.62)$, and pairs of psychiatrists and layinterviewers (kappa=.65). Procedural validity of the RDI was also evaluated by comparing the diagnosis made by a psychiatrist or lay interviewer using the RDI, with the diagnosis of the same patient made by a second psychiatrist using a standard research interview. The RDI was determined to correctly identify positive diagnoses in $80 \%$ of the cases. In addition, there was a high level of agreement 
between lay interviewers and psychiatrists in their diagnoses. The RDI is rarely used today because more sophisticated diagnostic interviews have been developed.

The next influential interview to be developed was the Schedule for Affective Disorders and Schizophrenia (SADS: Endicott \& Spitzer, 1978). The SADS was developed to yield diagnoses according to the Research Diagnostic Criteria (Spitzer et al., 1978). The SADS provides a detailed description of the current episode of illness as well as the subject's level of psychopathology and functioning during the week prior to the evaluation. Individual items were devised based on RDC criteria, and subsequently assigned to eight larger summary dimensions. Summary dimensions were developed to reduce the number of clinically meaningful measures on which to compare individual subjects over time or groups of subjects with each other.

Internal consistency for six of the eight Summary Scales on the SADS was .79 or above. The anxiety (.58) and thought disorder $(.47)$ summary scales were found to have low internal consistency. The SADS was field tested in two collaborative studies. In the first reliability study, a total of 150 psychiatric inpatients were jointly evaluated by pairs of interviewers. In a second study, 60 psychiatric inpatients who agreed to be interviewed twice were independently interviewed by a second interviewer within 24 
to 48 hours of the first interview. The intraclass correlation coefficients of reliability for the scaled items were calculated. The cumulative frequencies for both joint and test-retest evaluations indicated high levels of agreement for almost all of the scaled items, with $83 \%$ of the coefficients being .70 or greater for the joint evaluations, and $73 \%$ being .70 or greater for the testretest evaluations. The intraclass correlation coefficients of reliability for the summary scales were also high for both joint ( $r=.82$ to .99 ) and test-retest evaluations ( $r$ $=.49$ to .93$)$. The concurrent validity of the SADS was supported using correlations between the SADS summary scale Scores and two independent measures, the symptom Checklist90 (SCL-90: Derogatis, Lipman, \& Covp, 1973) and the Katz Adjustment Scales-Revised (Graham, 1973).

With the development of the DSM-III criteria, the National Institute of Mental Health (NIMH) Diagnostic Interview Schedule (DIS: Robins, Helzer, Croughan, \& Ratcliff, 1981) was constructed to include consideration of the DSM-III, Feighner, and RDC diagnostic systems. The DIS is a highly structured interview that was designed to be used by lay interviewers, in order to reduce the cost of data collection. In the original DIS, 43 DSM-III diagnoses were represented which included primarily Axis I adult diagnoses in an attempt to reduce the length of the interview. The DIS provides specific diagnoses and 
judgments about the severity of symptoms, their clustering in time, their frequency, and possible alternative explanations for their occurrence. The interrater reliability of the DIS was evaluated using 216 subjects who were interviewed twice, once by a lay interviewer and once by a psychiatrist. The resulting kappa values were greater than .50 for all three sets of diagnostic criteria (i.e., Feighner, RDC, and DSM-III). The lay interviewers' credibility was evaluated by examining the proportion of lay interviewers' positive diagnoses that were corroborated by the psychiatrists' diagnoses. Lay interviewers were found to agree with the psychiatrists' diagnoses on average for $75 \%$ of the diagnoses made across the three sets of diagnostic criteria.

With the revision of the DSM-III criteria in 1987, the Structured Clinical Interview for DSM-III-R (SCID: Spitzer, Williams, Gibbon, \& First, 1992) was developed to aid in the efficient gathering of assessment information. The SCID is a semi-structured interview that yields current and lifetime DSM-III-R diagnoses. It is administered by a clinician and includes an introductory overview followed by nine diagnostic modules, seven of which represent the major Axis I diagnostic classes. The initial semi-structured overview of the development and history of the presenting problem yields a tentative diagnosis which is then 
systematically assessed by structured questions in the diagnostic modules that include DSM-III-R criteria.

A reliability study of the SCID involved seven sites. Six sites were in the United States and included four psychiatric facilities, a health maintenance organization, and a site evaluating non-patient subjects from the community. A seventh included a research institution in Germany. The study was conducted in four phases. In Phase I, the primary objective was to update the SCID questions to correspond to the evolving DSM-III-R criteria. The SCID was tested in joint interviews in which two clinicians independently interviewed and rated the same subject. In Phase II, senior staff from all the sites were trained in the use of the SCID, and joint interviews were conducted. Phase III began with the selection and training of interviewers who all had advanced degrees in mental health. They then conducted a series of pilot interviews that were audiotaped and reviewed by the authors to provide feedback. Phase IV involved actual test-retest interviews at all the sites. Five hundred ninety-two subjects were interviewed, 390 were patients and 202 were non-patients. Sixteen of the 25 raters participated in the cross-site study, evaluating 98 cases that included both patients and non-patients. Kappa coefficients indicating rate of agreement between raters were in the fair to good range for most diagnostic categories. Mean kappas for current and 
lifetime diagnoses were above .60 , with a mean of .61 for current and .68 for lifetime diagnoses. For non-patients, agreement was considerably lower, with a mean kappa of .37 for current and .51 for lifetime diagnosis. Validity has also been supported in a study that compared DSM-III-R personality disorder diagnoses made with the SCID to diagnoses made by a panel of mental health professionals who relied on intra-panel consensus and inpatient ward observations over an extended period of time (skodol et al., 1988). Agreement was generally satisfactory, but higher for disorders defined by specific behaviors (e.g., antisocial and schizotypal personality disorders) than for those requiring a greater degree of inference (e.g., narcissistic and self-defeating personality disorders). Development of Interview Schedules for Specific Diagnoses With the recent expansions in the diagnostic criteria of DSM-IV, the need for specific purpose interview schedules that promote efficient data gathering for specific clinical disorders has become apparent. Interviews for specific psychiatric diagnoses have been developed for a variety of clinical disorders. Some examples include the Yale-Brown Obsessive Compulsive Scale (Goodman et al., 1989), the Comprehensive Drinker Profile (Miller \& Marlatt, 1984), and the Psychosocial Pain Inventory (Heaton, Lehman, \& Getto, 1980). One diagnostic category in need of a structured interview to aid in the differential diagnosis 
of its subtypes are the eating disorders. Two interview formats have been developed to standardize the collection of assessment information. These interviews are the Clinical Eating Disorder Rating Instrument (CEDRI: Palmer, Christie, Cordle, Davies, \& Kenrick, 1987), and the Eating Disorder Examination (EDE: Cooper \& Fairburn, 1987). Neither of these interviews was designed for use as a diagnostic interview. The only interview designed to provide eating disorders diagnoses according to DSM-III-R criteria is the Interview for the Diagnosis of Eating Disorders (Williamson, 1990). However, with the recent publication of the DSM-IV diagnostic criteria, all three interview schedules will be somewhat dated. Therefore, the purpose of the current study is develop a diagnostic interview based on the DSM-IV eating disorders criteria. One of the first interviews designed to assess eating disorder symptoms exclusively was the clinical Eating Disorder Rating Instrument (CEDRI: Palmer et al., 1987). The CEDRI is a semi-structured interview designed to be an observer rating scale. The first part of the inventory addresses the specific psychopathology of anorexia nervosa and bulimia nervosa. The remainder of the scale is concerned with general psychopathology often associated with eating disorders. The scale was initially piloted on a group of eight patients with primary eating disorders (four anorexics and four bulimics) and two controls (one obese 
and one normal subject). Four trained interviewers tape recorded interviews with two patients using the scale format, and two of the team also interviewed one control subject. All 10 tapes were then independently rated by the five raters, resulting in 50 ratings for each item on the scale. A second interrater reliability study was then carried out on the revised scale using an independent sample of 11 female subjects, 8 of whom were diagnosed with a primary eating disorder and 3 of whom were out-patient psychiatric controls. The audiotaped interviews were again rated independently, by five raters. In the first study, interrater reliability of the scale was found to be .70 or greater for 29 of 31 scale items (exceptions were reduced appetite $r=.59$, depressed $\operatorname{mood} r=.68$ ). In the second study, interrater reliability was found to be .70 or greater for 32 of 35 items contained in the interview (exceptions were use of drugs for weight control $r=.60$, average alcohol consumption $r=.66$, and abuse of drugs $r=.40$ ). Limitations of the CEDRI include its exclusive focus on the general features of anorexia nervosa and bulimia nervosa, without consideration of the specific criteria of a standard diagnostic system. In addition, reliability and validity data pertaining to the CEDRI are limited.

The Eating Disorder Examination (EDE: Cooper \& Fairburn, 1987) is a well-developed semi-structured clinical interview for assessing the symptoms of anorexia 
and bulimia nervosa. It has been revised 12 times, and its psychometric properties have been tested in many empirical studies (Fairburn \& Cooper, 1993). The four subscales of the EDE include the Restraint, Eating Concern, Shape Concern, and Weight Concern Subscales. The items derived from the interview are 23 symptom ratings made by the interviewer. For each item, there is at least one mandatory probe question and a number of subsidiary questions intended to assist the interviewer in making a rating. Items are rated based on the severity or frequency of a particular symptom. Preliminary items were selected through review of the anorexia and bulimia literature, and based on a series of unstructured interviews with anorexic and bulimic patients that provided descriptions of general behavior and attitudes. Once a list of preliminary items was identified, specific questions were devised for each item to help the interviewer make a rating. A coding scheme was also devised with anchor points appropriate to each individual item. Pilot interviews were then administered to anorexic, bulimic, and age-matched control subjects. On the basis of these interviews, redundant items were eliminated, items were simplified, and key terms were defined.

The interrater reliability of the EDE was evaluated, using three raters and 12 subjects. The three raters each conducted four EDE interviews and rated the recorded interviews of the other two raters. Subjects were nine 
bulimic women and three women with no eating disorder. The internal consistency of the subscales was found to be adequate to high with coefficient alpha ranging from .67 to .90 (Cooper, Cooper, \& Fairburn, 1989). Interrater reliability of all EDE items was found to be uniformly high with Pearson correlation coefficients ranging from .69 to 1.00 (Cooper \& Fairburn, 1987; Wilson \& Smith, 1989). The interrater reliability of the five EDE Subscales ranged from .83 to .99 (Rosen et al., 1990). Evidence for the concurrent and discriminant validity of the EDE has been reported for symptom severity (Fairburn \& Cooper, 1993).

The EDE was originally developed to assess the outcome of therapy and to evaluate the psychopathology of anorexia and bulimia nervosa. It has recently been expanded to enable the clinician to diagnose anorexia and bulimia nervosa using the diagnostic criteria of the DSM-IV. However, there have been no formal tests of the validity of the EDE as a tool for diagnosis of the eating disorders. In addition, there is no consideration of neither Binge-Eating Disorder nor the other diagnoses subsumed under Eating Disorder Not Otherwise Specified. Moreover, the EDE is based only on current symptomatology without the investigation of historical or developmental factors.

The Interview for the Diagnosis of Eating Disorders (IDED: Williamson, 1990) has been the only semi-structured interview for differential diagnosis of eating disorders as 
described in DSM-III-R since its development (see Appendix A). The original interview format contains four major categories: General Assessment and History, Anorexia Nervosa, Bulimia Nervosa, and Compulsive Overeating. After administration of the interview, interviewers are instructed to rate 19 symptoms related to the diagnostic criteria for anorexia nervosa, bulimia nervosa, and compulsive overeating. Each diagnostic category has a total score, which is the sum of all the ratings within each specific diagnostic category.

The psychometric properties of the IDED were assessed (Williamson, Davis, Norris, \& Van Buren, 1990) with a sample of five bulimics, five compulsive overeaters, five obese, and five normal subjects $(n=20)$. These subjects were interviewed using the IDED. Two weeks later, the subjects were re-interviewed, using the IDED, by a second interviewer, who did not have access to the results of the initial interview. The temporal stability of the instrument (over a two-week period of time), and the interrater reliability, proved to be more than adequate, with interrater reliability coefficients for each of the 19 ratings .86 or higher. The test-retest reliability coefficients for the total ratings for each of the subscales were also high (Anorexia Nervosa $r=.87$, Bulimia Nervosa $r=.94$, and Compulsive overeater $r=.94)$. Williamson et al. (1990) found $100 \%$ agreement for diagnoses made by 
pairs of interviewers. Concurrent validity of the IDED was also supported by the significant correlations found between the total scale scores of the IDED Subscales and several measures of related constructs.

The IDED has undergone two revisions since its initial development. The first revision of the IDED, the IDEDRevised (IDED-R), followed the pilot testing of the original interview schedule, and involved clarification of the wording of interview questions, and elimination of redundant items. The IDED-R was later modified (i.e., IDEDIII) to include the recently proposed Binge-Eating Disorder criteria. Pilot studies conducted of the IDED-III identified the need for the elimination of redundant items, and the development of interview questions that would aid in making the ratings for the Binge-Eating Disorder criteria. More recently, the revision of the DSM-III-R eating disorder diagnostic criteria in the recent publication of DSM-IV, has created a need for the revision of the IDED-III to accommodate these changes.

The purpose of the current study was to revise the IDED-III for the diagnoses of eating disorders according to DSM-IV diagnostic criteria, and to evaluate the psychometric properties of this revised interview protocol. With the publication of DSM-IV, existing interviews are now inadequate, and an interview that aids in the differential diagnosis of eating disorders has not yet been developed. 
Specifically, there is not a diagnostic interview available to differentiate between Anorexia Nervosa, Bulimia Nervosa, Binge-Eating Disorder, Eating Disorder Not Otherwise Specified, and normals, based upon the new criteria set forth by DSM-IV. The IDED-IV was designed to enable differential diagnosis of the DSM-IV eating disorders, and to enable the detailed assessment of typical eating disorder symptoms that could be targeted in treatment. The goals of this study were to revise the IDED-IV to reflect the DSM-IV eating disorder criteria and to evaluate the interview's psychometric properties. This study was conducted in three phases. Phase I involved the development of the IDED-IV, Phase II was a reliability study of the IDED-IV, and Phase III was a validity study of the IDED-IV. 
Strategy for the Development of the IDED-IV

Phase I: Development of the IDED-IV

Changes in structure from an earlier version of the

IDED. Several revisions were made to the IDED-III to

enhance its utility as a diagnostic interview, as well as

to make it applicable to DSM-IV eating disorder criteria.

The revisions were made to the IDED-IV based on the results

of a pilot study conducted with the IDED-III by three pre-

doctoral and three post-doctoral interviewers in four

different treatment facilities. Interviewers were three

pre-doctoral graduate students and three unlicensed

psychologists who had four to seven years of specialized

training in the assessment and treatment of eating

disorders, which included administration of earlier

versions of the IDED. Interviewers were all asked to

provide feedback regarding suggested modifications to the

IDED that would enhance its ability for providing reliable

diagnoses and for assessing symptom severity.

Several revisions were made to the IDED to enhance its

utility as a diagnostic interview and its ability to assess symptom severity for the eating disorders as defined by DSM-IV eating disorder criteria. First, the portion of the interview inquiring about demographic information was expanded to include questions identifying medical and mental health providers, previous treatment history, current medications, and insurance information. Second, the 
instructions for administering the IDED-IV were expanded to improve standardization in administration of the interview by providing interviewers with a specific set of guidelines for conducting the interview. Third, the General Assessment and History section in the IDED-III was expanded in the IDED-IV to include a section enabling systematic inquiry regarding an individual's history of eating disorder symptoms, and a separate section on the current status of eating disorder symptoms which includes the assessment of eating patterns, medical problems, and family information. Fourth, the diagnostic subsections were revised to include headings cuing the interviewer regarding the rating being made at the end of a set of interview questions. Fifth, ratings and related questions were revised to reflect DSM-IV eating disorder criteria. In addition, ratings were condensed from a 7-point to a 5-point likert scale, and the descriptors for the 5 points were modified to be more clear and concise. Sixth, specific instructions were included throughout the interview to aid in the completion of the diagnostic checklist at the end of the interview. Also, within the diagnostic checklist, specific instructions were included to assist interviewers in making diagnoses.

Format and content of the IDED-IV. The interview format of the IDED-IV contains 10 major sections: Demographic Information, Instructions for Administration, 
History of Eating Disorder symptoms, Current status of Eating Disorder Symptoms, Questions for Diagnosing Anorexia Nervosa, Questions for Diagnosing Binge-Eating Disorder, Questions for Diagnosing Bulimia Nervosa, Questions for Eating Disorder Not Otherwise Specified, Other Eating Problems, and Diagnostic Checklists for the Eating Disorders. The IDED-IV begins with an overview section that follows the general structure of a clinical diagnostic interview. First, basic demographic information is obtained. This section is followed by an open-ended history of eating-disorder symptoms, a description of the chief complaint, and general questions about current functioning. The semi-structured overview is followed by fullystructured diagnostic sections that systematically inquire about specific symptoms. The diagnostic sections each begin with a heading intended to cue the interviewer with regard to the DSM-IV diagnostic criterion being rated at the end of each set of questions. Twenty ratings are made on a 5point scale, on which either frequency or severity are rated. A rating of three or more on each of the symptoms is the operational definition for concluding that the subject has endorsed the presence of that diagnostic symptom. Due to the overlap in symptoms across eating disorder diagnostic categories, some ratings have been replaced with instructions for transferring information to the diagnostic checklist found at the end of the interview schedule. In 
order to reduce the redundancy in the interview's content and any related inconvenience to the subject, the interviewer is responsible for completing the diagnostic checklist according to the instructions provided throughout the interview and summarized within the diagnostic checklist. To promote the flow of the interview, it is recommended that transfer of information from the interview to the diagnostic checklist be completed immediately after the interview has been completed. Phase II: Reliability of the IDED-IV

The second phase of the study evaluated the internal consistency and the interrater reliability of the IDED-IV. The entire sample of subjects was utilized to determine the interview's internal consistency using coefficient alpha and item-total correlations. The interrater reliability was evaluated with a subsample of the initial pool of subjects. One of 11 interviewers with specialized training in the administration of the IDED-IV conducted the first diagnostic interview. Eighty-two of the 176 subjects who agreed to have their clinical interviews audiotaped participated in this portion of the study. Two of the 11 original interviewers, who were blind to the results of the first set of diagnostic interviews, conducted reliability checks by listening to the audiotaped interviews of the 
first interviewers. A kappa coefficient was utilized to determine the rates of agreement between pairs of interviewers.

\section{Phase III: Validity of the IDED-IV}

The validity of the diagnoses derived in Phase II using the IDED-IV was evaluated in Phase III of the current study. A subset (i.e., 147 of the 176) of the initial sample of subjects was used in the validation phase of the current study. Based on the diagnostic profiles of the subjects derived through administration of the IDED-IV, subjects were assigned to one of five groups (i.e., three clinical and two control). The three clinical groups included subjects with Anorexia Nervosa $(\underline{n}=22)$, Bulimia Nervosa $(\underline{n}=27)$, or Binge-Eating Disorder $(\underline{n}=44)$. The use of the term eating disorder subjects in this text will denote individuals classified in one of the three clinical groups. The two control groups included obese $(\underline{n}=25)$ and Normal Weight Control $(\underline{n}=29)$ subjects. Obesity was not included in the eating disorder classification due to its status as a medical condition rather than a mental illness. The convergent and discriminant validity of the IDEDIV was evaluated by comparatively examining subjects' scores on self-report measures of related and unrelated constructs. Toward this end, Pearson correlation coefficients were used to examine the intercorrelations between the IDED-IV Subscales and the self-report measures 
prior to making group comparisons. Several predictions were made with regard to the correlations within the IDED-IV Subscales (see Table 5) and between the IDED-IV Subscales and self-report measures of related and unrelated constructs (see Table 6). Due to the significant overlap in eating disorder symptomatology, it was predicted that the IDED-IV Subscales would be intercorrelated. As seen in Table 5, it was predicted that the Anorexia Nervosa and Bulimia Nervosa Subscales would be moderately positively correlated due to the new diagnostic subtypes having overlapping symptoms that assess restrictive eating, binge eating, purging, and body image disturbance, although Anorexic subjects would primarily engage in restrictive eating and Bulimic subjects in binge eating. It was also predicted that the IDED-IV Bulimia Nervosa and Binge-Eating Disorder subscales would be moderately positively correlated given their overlap with regard to binge eating and loss of control symptomatology, and their dissimilar focus on the presence versus the absence of compensatory behavior. Finally, a low positive correlation was predicted between the Anorexia Nervosa and Binge-Eating Disorder Subscales given that the former emphasizes restrictive eating while the latter emphasizes binge eating, although individuals with each of these disorders may have either or both symptoms to varying degrees. 
Table 5

Predicted Intercorrelations Between the IDED-IV Subscale Totals

\begin{tabular}{llll}
\hline & \multicolumn{3}{c}{ IDED-IV Subscales } \\
& AN & BED & BN \\
IDED-IV Subscales & & & \\
AN & -- & low & $\bmod +$ \\
BED & -- & - & $\bmod +$ \\
BN & -- & - & - \\
\hline
\end{tabular}

Note. IDED-IV = Interview for the Diagnosis of Eating Disorders-Fourth Edition, $\mathrm{AN}=$ Anorexia Nervosa, $\mathrm{BED}=$ Binge Eating Disorder, $\mathrm{BN}=$ Bulimia Nervosa. $* \mathrm{p}<.0001$ 
Table 6

Predicted Intercorrelations Between the IDED-IV Subscale Totals and scores on Self-Report Measures of Eating Disorder symptoms

IDED-IV Subscales

Self-Report

AN

$\mathrm{BN}$

BED

Measures

EAT Factors

$\begin{array}{llll}\text { Dieting } & \mathrm{hi}+ & \bmod + & \text { low }+ \\ \text { Oral Control } & \mathrm{hi}+ & \bmod +\end{array}$

BULIT-R Factors

$\begin{array}{lll}\text { Binge Eating } & \text { low to mod }+\mathrm{hi}+ & \mathrm{hi}+ \\ \text { Rad wt Loss Meas } & \text { low to mod }+\bmod \text { to } \mathrm{hi}+ & \text { low }+ \\ \text { Vomiting } & \text { low to mod }+\bmod \text { to } \mathrm{hi}+ & \text { low }+ \\ \text { Lax/Diur Abuse } & \text { low to mod }+\bmod \text { to } \mathrm{hi}+ & \text { low }+ \\ \text { Exercise } & \text { low to mod }+\bmod \text { to } \mathrm{hi}+ & \text { low }-\end{array}$

EDI-II Subscales

$\begin{array}{llll}\text { Drive for Thin } & \text { hi }+ & \text { hi }+ & \bmod + \\ \text { Body Dissatis } & \bmod + & \text { hi }+ & \text { hi }+\end{array}$

BIA Subscales

Current Body size mod + hi + hi +

Ideal Body size low - low - mod +

Note. IDED-IV = Interview for the Diagnosis of Eating Disorders-Fourth Edition, $A N=$ Anorexia Nervosa, $B N=$ Bulimia Nervosa, $\mathrm{BED}=$ Binge Eating Disorder, $\mathrm{EAT}=$ Eating Attitudes Test, BULIT-R = Bulimia Test-Revised, EDI-II Eating Disorder Inventory-Second Edition, BIA = Body Image Assessment Procedure. Rad $=$ Radical; $W t=$ Weight; Meas $=$ 
Measures; $\operatorname{Lax}=$ Laxative; Diur $=$ Diuretic; Thin $=$ Thinness; Dissatis = Dissatisfaction. All correlations greater than .27 are significantly greater than. $*$ p $<.0001$. 
Table 6 summarizes the predicted correlations between the IDED-IV Subscales and self-report measures of related and unrelated constructs. Predictions regarding subjects' performance on the dependent measures were made based on the diagnostic criteria for the eating disorders in DSM-IV. Ranges were predicted for some of the dependent measures given the influence of diagnostic subtypes on the primary eating disorder categories in DSM-IV. To illustrate, Anorexia Nervosa has historically been defined as a disorder characterized by extreme dieting, low body weight, fear of weight gain, body image disturbance, and amenorrhea consistent with the Anorexia Nervosa Restricting Type in DSM-IV. However, with the addition of the BingeEating/Purging subtype in DSM-IV, the symptom profile is more complex, and further diversifies the group of subjects identified as having Anorexia Nervosa. Thus, the accuracy of predictions made regarding group membership would largely depend on subject characteristics represented in a particular sample. Therefore, on dependent measures that would be most largely affected by the diagnostic subtyping within DSM-IV, a range for the correlation between scores on the IDED-IV Subscales and dependent measures was provided.

As depicted in Table 6, it was predicted that subjects' scores on the IDED-IV Anorexia Nervosa Subscale would be highly positively correlated with the EAT Dieting 
Factor, EAT oral Control Factor, and EDI-II Drive for Thinness Subscale given Anorexic subjects low body weight and extreme dieting behavior. Moderate positive correlations were predicted between the IDED-IV Anorexia Nervosa Subscale and both the EDI Body Dissatisfaction Subscale and the BIA for current Body Size due to Anorexic subjects' tendency to deny and/or minimize their concern with their body weight. Low to moderate positive correlations were predicted for the all five of the BULIT-R Factors due to the variable performance expected on these measures depending on the proportion of subjects with the Binge-Eating/Purging versus the Restricting subtype. Finally, a low negative correlation was predicted between the IDED-IV Anorexia Nervosa Subscale and the BIA for Ideal Body Size, given Anorexic subjects' tendency to idealize below average body weights and shapes.

With regard to the IDED-IV Bulimia Nervosa Subscale, high positive correlations were predicted with the BULIT-R Binge-Eating Factor, EDI-II Drive for Thinness subscale, EDI-II Body Dissatisfaction Subscale, and the BIA for Current Body size. These high correlations were predicted based on Bulimic subjects' high likelihood to engage in binge eating, to be highly motivated to lose weight and resort to extreme weight loss measures, and to have a body image disturbance for which would involve perceiving themselves as being larger than they really are. Moderate 
to high correlations were predicted between the IDED-IV Bulimia Nervosa Subscale and BULIT-R Factors evaluating compensatory behaviors including the Radical Weight Loss Measures, Vomiting, Laxative/Diuretic Abuse, and Exercise Factors. The term compensatory behavior is used in DSM-IV to refer to any behaviors used by eating disorder subjects to rid their bodies of food. Within the definition of compensatory behaviors, vomiting, laxative/diuretic abuse, excessive exercise, and strict fasting are included. The term purging is used only to denote vomiting and laxative/diuretic abuse. Moderate to high correlations were predicted on measures of compensatory behaviors due to this relationship depending on the number of purging versus nonpurging Bulimics recruited in the current sample. Moderate positive correlations were predicted between the IDED-IV Bulimia Nervosa Subscale and the EAT Dieting and Oral Control Factors due to Bulimic subjects' tendency to engage in strict dieting prior to binge eating. Finally, a low negative correlation was predicted for the BIA for Ideal Body size given Bulimics tendency to idealize low body weights and shapes. Predicted correlations for the IDED-IV Binge-Eating Disorder subscale included high positive correlations with the BULIT-R Binge-Eating Factor, the EDI Body Dissatisfaction Factor, and the BIA for Current Body size. These high correlations were anticipated in light of 
subjects with this disorder being characterized by high levels of binge eating, marked distress regarding binge eating, and the absence of a body image disturbance. Moderate positive correlations were predicted between the IDED-IV Binge-Eating Disorder Subscale and both the EDI Drive for Thinness Subscale and the BIA for Ideal Body Size. It was anticipated that due to their marked distress regarding their binge eating and their lack of a body image disturbance, that Binge-Eating Disorder subjects would report a moderate motivation for dieting and a realistic ideal body size. A moderate negative correlation was predicted between the IDED-IV Binge-Eating Disorder Subscale and the EAT Oral Control Factor given subjects' report of the frequent loss of control during eating. Low positive correlations were predicted with the EAT Dieting Factor, BULIT-R Radical Weight Loss Measures Factor, BULIT$\mathrm{R}$ Vomiting Factor, and the BULIT-R Laxative/Diuretic Abuse Factor. This prediction was made in light of the possibility that some Binge-Eating Disorder subjects might resort to sporadic compensatory behavior to relieve themselves of physical discomfort following a binge, and/or due to the likelihood that Bulimia Nervosa subjects would also score high on the IDED-IV Binge-Eating Disorder Subscale, and thereby influence the correlation between the Binge-Euting Disorder Subscale and measures of compensatory 
behavior. A low negative correlation was predicted with the BULIT-R Exercise Factor due to individuals with this disorder generally having a strong aversion to exercise.

Given the predictions that the IDED-IV Subscales would be intercorrelated with each other as well as with selfreport measures of related constructs, a canonical correlation was used to further evaluate these relationships by considering all potential relationships simultaneously while controlling for the Type I error rate. In light of the development of diagnostic subtypes within DSM-IV (i.e., Anorexia Nervosa, Restricting Type or BingeEating/Purging Type; Bulimia Nervosa, Purging or Nonpurging Type), it was predicted that subjects might have elevations on more than one subscale of the IDED-IV with comparable elevations on measures of related constructs.

The convergent and discriminant validity of the IDEDIV was further examined by comparing the performance of the diagnostic groups identified by the IDED-IV on dependent measures of related and unrelated constructs, respectively. Dependent measures were selected based on their correspondence to DSM-IV eating disorder criteria, and therefore assessed dieting behavior, body image, binge eating, and compensatory behaviors. Given the overlap in symptomatology across eating disorder groups, it was predicted that subjects would score similarly on measures of common symptoms and thus provide evidence for convergent 
validity, and dissimilarly on measures of differentiating symptoms and thus provide evidence for discriminant validity.

Thus, it was predicted that Anorexic and Bulimic subjects would obtain similar scores on measures of dieting and body image disturbance, and dissimilar scores on binge eating and purging. It was also predicted that Bulimic and Binge-Eating Disorder subjects would obtain similar scores on measures of binge eating, and dissimilar scores on measures of dieting, compensatory behavior, and body image disturbance. Finally, for the Anorexia Nervosa and BingeEating Disorder groups, it was predicted that subjects would score dissimilarly on measures assessing dieting behavior, body image disturbance, binge eating, and compensatory behaviors.

In order to determine whether significant differences existed between the diagnostic groups, a multivariate analysis of variance (MANOVA) was used to control for the intercorrelations between the IDED-IV Subscales and dependent measures and the possibility of a Type I error. Once significant differences were found between the groups, univariate analyses of variance (ANOVAs) and Scheffe post-hoc comparisons were used to identify group differences based on an alpha level of $\mathfrak{p}<.05$. 
Method

\section{$\underline{\text { Subjects }}$}

Initial sample. One hundred seventy-six subjects participated in the study. The sample was composed of 162 women and 14 men. Ninety-six clinical subjects were found to meet criteria for one of several DSM-IV eating disorder diagnoses. Clinical subjects were recruited from either a private psychiatric hospital, a large research center, or from a psychology clinic on campus. Advertisements in the local newspaper and on the evening news program were used to recruit both clinical and nonclinical subjects to the psychology clinic on campus. A total of eighty nonclinical subjects were recruited from the community, as well as from undergraduate classes at Louisiana state University. Consent forms explaining the purpose and procedure of the study were signed by subjects prior to their participation (see Appendix A).

The clinical group included 90 women and 6 men, with a mean age of 33.7 years $(\underline{S D}=14.3)$ (see Table 7 ). The ethnicity of the clinical sample included $88.5 \%$ Caucasian $(\underline{n}=85)$ and $11.5 \%$ African $(\underline{n}=11)$ Americans. The Hollingshead two factor index of SES indicated that the majority of subjects ( $92.3 \%$ ) were from middle to upper middle class backgrounds. The average height was $1.65 \mathrm{~m}$ (SD $=.07 \mathrm{~m}$ ), with a range from 1.50 to $1.88 \mathrm{~m}$. The average 
Table 7

Descriptive variables for clinical and nonclinical subjects in the initial sample

\section{Group}

$\begin{array}{ccc}\text { Variables } & \text { Clinical } & \text { Nonclinical } \\ & \underline{\mathrm{n}}=96 & \underline{\mathrm{n}}=80\end{array}$

Age

$33.7(14.3)$

$30.7(15.2)$

Ethnicity

Caucasian

$88.5 \%$

$71.2 \%$

African-American

$11.5 \%$

$20.0 \%$

other Minority

$0.0 \%$

$8.7 \%$

Socioeconomic status

Middle to Upper Middle $92.3 \%$

$70.0 \%$

Height

$1.65(.07)$

$1.65(.07)$

Weight

$88.50(37.80)$

$83.40(31.70)$

Body Mass Index

$29.30(11.80)$

$27.30(10.10)$

Percent Obese

$48.0 \%$

$31.0 \%$

Treatment History

For Eating Disorder $\quad 46.0 \%$

$3.7 \%$

Psychological Problems 18.0\%

$2.5 \%$

Note. Means are shown with standard deviations in parentheses. 
weight was $88.5 \mathrm{~kg}(\underline{\mathrm{SD}}=37.8 \mathrm{~kg})$, the range being from 37.8 to $237.5 \mathrm{~kg}$. The mean Body Mass Index for the clinical subjects was $29.3(\underline{S D}=11.8)$. Body Mass Index was calculated by multiplying subjects' weights by .45 in the numerator and dividing by the subjects' heights multiplied by .025 in the denominator. Subjects with Body Mass Indices at 30.0 or higher were classified as being obese. By this standard, forty-eight percent of the clinical subjects were obese. Of the clinical subjects who were obese, $82 \%$ were Binge-Eating Disorder subjects and $18 \%$ were Bulimia Nervosa subjects.

A significant proportion of the clinical subjects had been treated for either eating disorder (46\%) and/or other psychological problems (18\%). Subjects' current eating disorders diagnoses were determined through administration of the Interview for the Diagnosis of Eating Disorders-IV. Table 6 summarizes the diagnoses in this sample. Subjects also reported problems with night-binge eating (25.3\%), tasting (i.e., chewing and spitting out food before swallowing) (4.2\%), or a combination of these problems $(1.1 \%)$.

The nonclinical group included 72 women and 8 men, with a mean age of 30.7 years $(\underline{S D}=15.2)$. The ethnicity of the sample included $71.2 \%$ Caucasian $(\underline{n}=57), 20.0 \%$ African-American $(\underline{n}=16)$, and $8.7 \%(\underline{n}=7)$ other minority Americans. The Hollingshead two factor index of SES 
indicated that the majority $(70.0 \%)$ of subjects were from middle to upper middle class backgrounds. The average height was $1.65 \mathrm{~m}(\underline{\mathrm{SD}}=.07 \mathrm{~m})$ with a range from 1.52 to $1.83 \mathrm{~m}$. Subjects' average weight was $83.4 \mathrm{~kg} \quad(\underline{S D}=31.7$ $\mathrm{kg})$, the range being from 46.5 to $175 \mathrm{~kg}$. The mean Body Mass Index was $27.3(\underline{S D}=10.1)$. Thirty-one percent of the nonclinical subjects were obese. A small proportion of the nonclinical subjects had been treated for either eating disorder (3.7\%) and/or other psychological problems (2.5\%). only one nonclinical subject reported a problem with nightbinge eating ( $1.2 \%)$, and none of the nonclinical subjects reported problems with tasting. No other aberrant eating patterns were reported.

Subjects' clinical versus nonclinical group status was based on their responses to the IDED-IV. The IDED-IV provides interviewers with specific rules for determining DSM-IV eating disorder diagnoses. Figures 1 through 3 outline the rules for making Anorexia Nervosa, Binge-Eating Disorder, and Bulimia Nervosa diagnoses as well as their subclinical variations which are subsumed under the Eating Disorder Not Otherwise Specified (ED NOS) diagnosis. To be diagnosed with a particular eating disorder, subjects must have met all the criteria for a given disorder. Once meeting the criteria for a DSM-IV eating disorder, decisions were made regarding the subtype that described 
CRITERIA

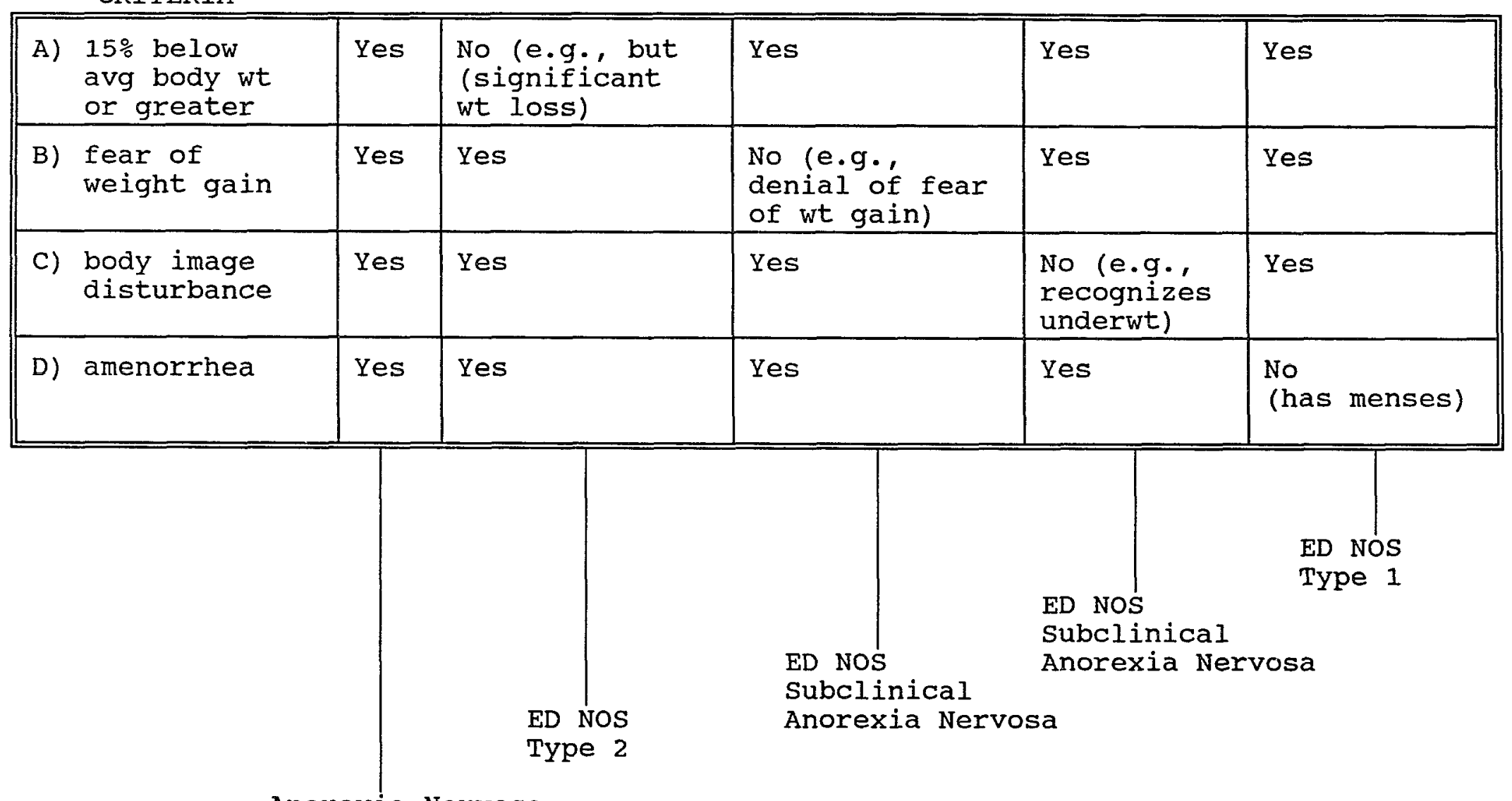

Anorexia Nervosa

Fiqure 1. Decision Tree for Making DSM-IV Anorexia Nervosa diagnosis and its Subclinical Variations Subsumed under Eating Disorder Not otherwise Specified (ED NOS). Avg = average Wt $=$ weight 
CRITERIA

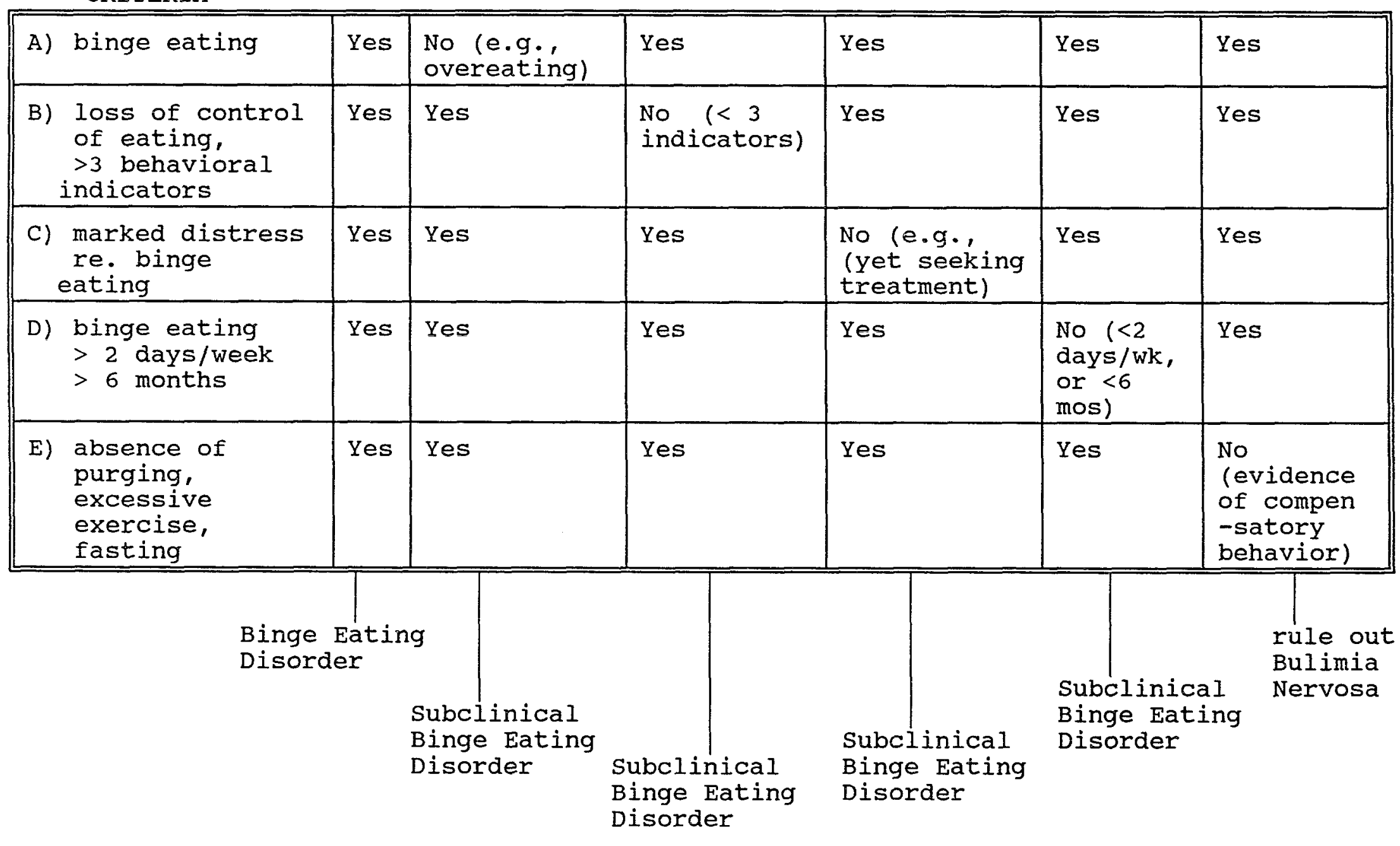

Figure 2. Decision Tree for Making DSM-IV Binge Eating Disorder Diagnosis and its Subclinical Variations Subsumed under Eating Disorders Not otherwise Specified (ED NOS). G 
CRITERIA

\begin{tabular}{|c|c|c|c|c|c|}
\hline A) & binge eating & Yes & $\begin{array}{l}\text { No (e.g. } \\
\text { overeating) }\end{array}$ & Yes & Yes \\
\hline B) & $\begin{array}{l}\text { compensatory behavior } \\
\text { (purging, exercise, } \\
\text { fasting) }\end{array}$ & Yes & Yes & No & Yes \\
\hline C) & $\begin{array}{l}\text { binge/purge cycle } \\
>2 \mathrm{x} / \mathrm{wk} \&>3 \text { mos }\end{array}$ & Yes & Yes & $\begin{array}{l}\text { Yes (binge } \\
\text { eating only) }\end{array}$ & $\begin{array}{l}\text { No }(<2 \mathrm{x} / \mathrm{wk} \text {, } \\
\text { or }<3 \text { mos })\end{array}$ \\
\hline & body image disturbance & Yes & Yes & Yes & Yes \\
\hline E) & $\begin{array}{l}\text { absence of Anorexia } \\
\text { Nervosa }\end{array}$ & Yes & Yes & Yes & Yes \\
\hline
\end{tabular}

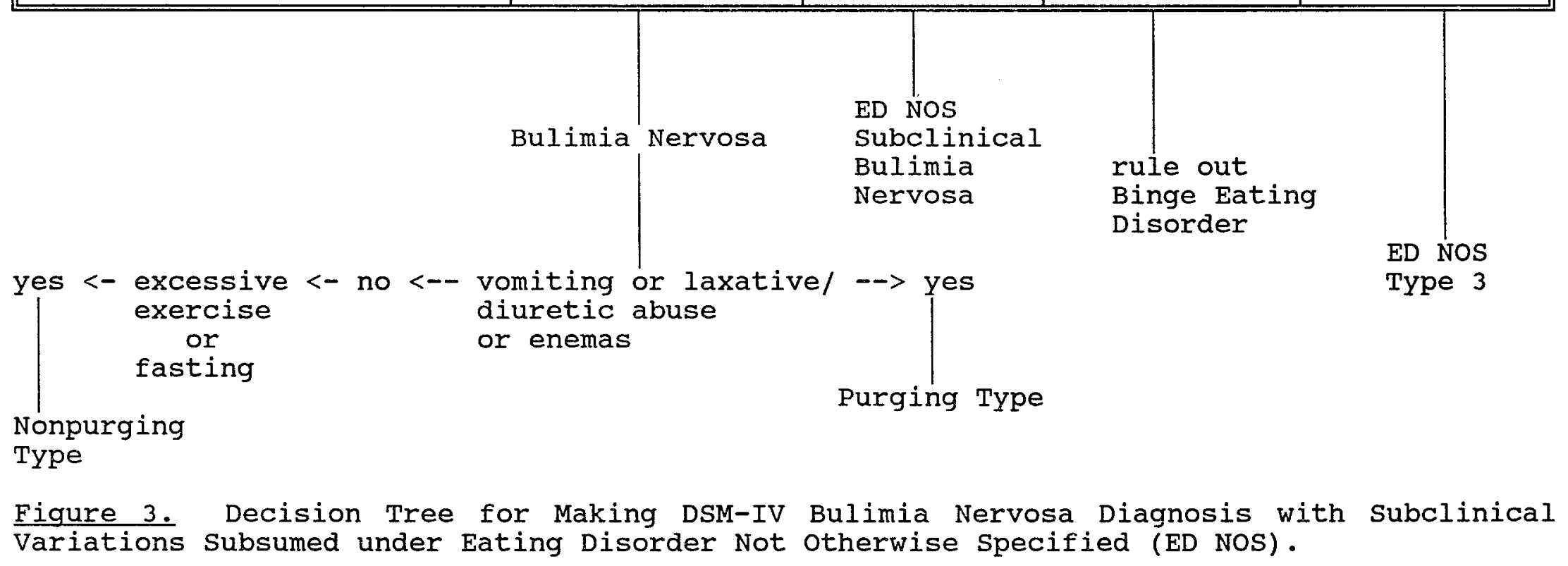

(figure con'd) 
CRITERIA

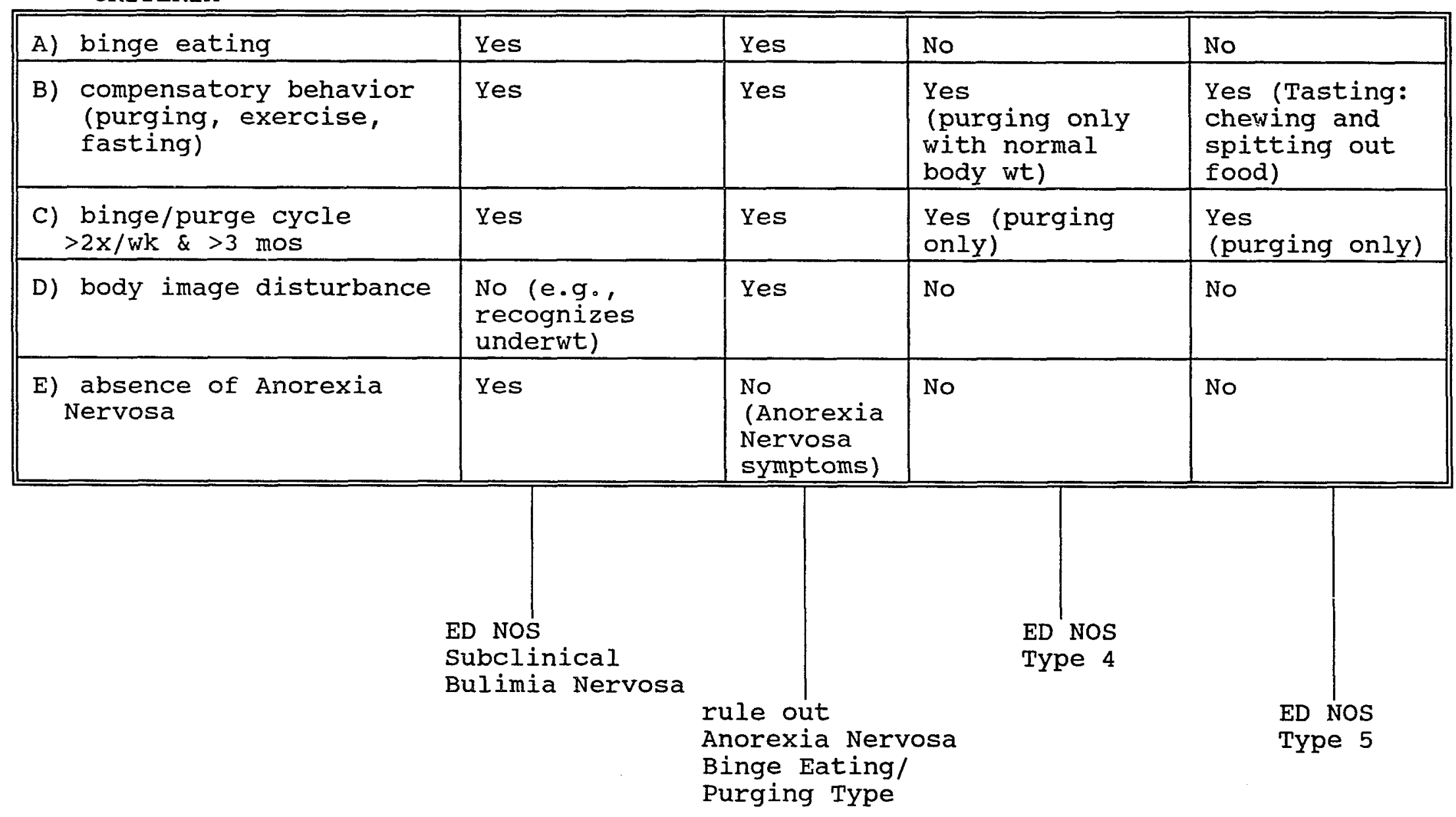


the subjects' symptomatology when applicable. When subjects met all but one of a given set of diagnostic criteria, interviewers made Eating Disorder Not Otherwise Specified diagnoses based on guidelines specified in Figures 1 through 3 . In addition, in Figure 2 , two variations on purging behavior are described which are included in the Eating Disorder Not Otherwise Specified category as Types 4 and 5. Table 8 summarizes the eating disorders diagnoses found for the current sample.

Once subjects were identified as being in the clinical or nonclinical group, chi square analyses were conducted to determine whether the groups differed with respect to sex, race, or socioeconomic status. No significant differences were found for sex or socioeconomic status. The groups did, however, differ with respect to race $\underline{x}^{2}(2, \underline{N}=176)=$ $12.09, \mathrm{p}<.002$. The nonclinical group had more AfricanAmerican and other minority subjects than the eatingdisorder clinical group.

A MANOVA was used to compare the clinical and nonclinical groups with regard to age and Body Mass Index. A MANOVA was utilized to control for intercorrelations between the variables, as well as the Type I error rate. The groups were compared on their BMI index values due to the inclusion of height and weight in the calculation of 
Table 8

Eating Disorder Diagnoses in the Clinical Group for the Initial Pool of Subjects

Diagnosis

Freq

$\%$

Anorexia Nervosa

Restricting Type

7

7.3

Binge-Eating/Purging Type

7

7.3

Bulimia Nervosa

Purging Type

17

17.7

Nonpurging Type

2

2.1

Eating Disorder Not Otherwise Specified

Anorexia Nervosa with menses

1

1.0

Anorexia Nervosa with normal weight

0

0.0

Bulimia Nervosa with infrequent b-p cycle

3.1

Normal Weight Individual who purges 3

3.1

Tasting

0

0.0

Binge Eating Disorder

38

39.6

Subclinical Anorexia Nervosa-Restricting

7

7.3

Subclinical Bulimia Nervosa-Purging

4

4.2

Subclinical Bulimia Nervosa-Nonpurging

1

1. 0

Subclinical Binge Eating Disorder

6

6.3

Total

96

100.0

Note. $b-p=b i n g e-p u r g e$. 
the BMI. Using an alpha level of .05, no significant differences were found between the two groups with respect to age or BMI.

Validation sample. For the validity portion of the study five diagnostic groups were formed based on subjects' responses to the IDED-IV in order that group comparisons could be made. The five diagnostic groups included three clinical groups (i.e., Anorexia Nervosa, Bulimia Nervosa, and Binge-Eating Disorder), and two control groups (i.e., Obese Control and Normal Weight Control). Subjects were assigned to one of the three clinical groups based on one of two conditions. Subjects either met full DSM-IV diagnostic criteria for a particular eating disorder or met the majority of the criteria but had some criteria that were not met due to being at subthreshold levels (e.g., meeting full criteria for Anorexia Nervosa except weight requirement, meeting full criteria for Bulimia Nervosa except binge eating less than twice per week). Support for the inclusion of eating disorder subjects with subclinical symptoms is provided by findings that a high percentage (33 to $46 \%$ ) of subclinical eating disorder females eventually meet full criteria for an eating disorder, and/or have persistent eating disorder symptoms with low rates of recovery at two- and four-year follow-up (Herzog, Hopkins, \& Burns, 1993; Bunnell, Shenkur, Nussbaum, Jacobson, \& Cooper, 1990; Yager, Landsverk, \& 
Edelstein, 1987). Authors in these studies concluded that the DSM-III-R diagnostic criteria may have been too restrictive, and that subclinical diagnoses may largely be an artifact of the timing of the diagnostic interview. Furthermore, individuals may have been either in an improved state, or in the process of developing a full disorder at the time of intake. Scarano \& Kalodner-Martin (1994) add that the fundamental difference among individuals with eating disorders who meet diagnostic criteria and individuals with milder forms of eating disorders is a matter of degree and not kind. In fact, even individuals with less severe eating disorder symptoms may still experience substantial distress and/or impairment (Herzog et al., 1993).

Thus, in the validation study, the Anorexia Nervosa group included not only individuals meeting full diagnostic criteria for Anorexia Nervosa Restricting Type, Anorexia Nervosa Binge-Eating/Purging Type, Anorexia Nervosa with Menses (i.e., Eating Disorder Not Otherwise Specified, Type 1), and Anorexia Nervosa with less than below average body weight (Eating Disorder Not otherwise Specified, Type 2), but also Subclinical Anorexia Nervosa subjects who met all criteria except for the fear of weight gain (i.e., possibly due to denial) or the body image disturbance (i.e., due to recognizing underweight status) criteria. 
Moreover, the Bulimia Nervosa group included subjects who obtained IDED-IV diagnostic profiles consistent with Bulimia Nervosa Purging Type, Bulimia Nervosa Nonpurging Type, Bulimia Nervosa with an infrequent binge-purge cycle (i.e., Eating Disorder Not Otherwise Specified, Type 3), or some subclinical variation. Subclinical Bulimia Nervosa subjects were those who met the majority of the diagnostic criteria except for not reporting binge eating (i.e., describe overeating), or not having a body image disturbance (i.e., possibly due to denial or minimization of symptoms). Subjects who were included in the BingeEating Disorder group included those individuals meeting full DSM-IV diagnostic criteria or some subclinical variation. Subclinical Binge-Eating Disorder patients were those who: did not report binge eating (i.e., describe overeating), had less than three behavioral indicators of loss of control during eating, denied experiencing marked distress regarding binge eating (i.e., yet were seeking treatment), or engaged in binge eating for less than twice per week or for less than three months.

Subjects who did not meet any of the DSM-IV criteria for an eating disorder as determined by the IDED-IV were assigned to an Obese or Normal Weight Control Group based on their Body Mass Index. The Body Mass Index was used as the criterion to determine control group membership due to its inclusion of the consideration of both height and 
weight in determining subjects' weight status. Individuals with a Body Mass Index of 30 or greater were assigned to the Obese group, while individuals with a Body Mass Index of 20 to 25 were included in the Normal Weight control group.

Table 9 summarizes the descriptive characteristics of the subjects in the five diagnostic groups. A MANOVA used to examine the groups on the basis of age, weight, height, and body mass index found significant differences across group, $\mathrm{F}(24,409)=7.02 \mathrm{p}<.0001$, using Wilk's criterion. Univariate analyses of variance with Sheffe post-hoc comparisons were used to evaluate group differences.

Group differences were found for age in that subjects in the Anorexia Nervosa, Bulimia Nervosa, and Normal Control groups had comparable ages, while subjects in the Binge-Eating Disorder and obese Control group were of comparable ages. In addition, Bulimic subjects were significantly heavier in weight and had higher body mass indices than Anorexic subjects, and Normal Weight Control subjects had body weights and body mass indices that were intermediate between the two groups but were not significantly different from either group. Moreover, BingeEating Disorder and Obese subjects had similar weights and body mass indices. No differences in height were found across the groups. 
Table 9

Descriptive Variables for Clinical subjects in the Validation Sample

\begin{tabular}{|c|c|c|c|c|c|}
\hline \multirow[b]{2}{*}{ Variable } & \multirow[b]{2}{*}{$\mathrm{BN}$} & \multicolumn{2}{|c|}{ Group } & \multirow[b]{2}{*}{ NORM } & \multirow{3}{*}{$\underline{F}$} \\
\hline & & BED & OBES & & \\
\hline $\begin{array}{l}\text { e } \quad A N \\
\underline{n}=22\end{array}$ & $\underline{n}=27$ & $\underline{\mathrm{n}}=44$ & $\underline{\mathrm{n}}=21$ & $\underline{\mathrm{n}}=29$ & \\
\hline $26.18^{a}$ & $27.33^{a}$ & $42.14^{b}$ & $45.68^{b}$ & $23.86^{a}$ & $19.97 *$ \\
\hline$(10.62)$ & $(10.32)$ & $(13.98)$ & $(14.38)$ & $(8.50)$ & \\
\hline WEIGHT $50.18^{a}$ & $80.91^{b}$ & $114.50^{\mathrm{C}}$ & $121.91^{\mathrm{C}}$ & $66.18^{a b}$ & $40.11 *$ \\
\hline$(6.68)$ & $(33.21)$ & $(30.63)$ & $(28.72)$ & $(7.17)$ & \\
\hline HEIGHT & 1.64 & 1.66 & 1.67 & 1.65 & 1.02 \\
\hline$(.06)$ & $(.08)$ & $(.08)$ & $(.07)$ & $(.07)$ & \\
\hline $16.77^{a}$ & $27.26^{b}$ & $37.52^{c}$ & $40.55^{c}$ & $22.28^{a b}$ & $44.57 *$ \\
\hline$(1.90)$ & $(10.32)$ & $(9.09)$ & $(9.43)$ & $(1.77)$ & \\
\hline
\end{tabular}

Note. $A N=$ Anorexia Nervosa; $B N=$ Bulimia Nervosa; $B E D=$ Binge Eating Disorder; OBES = Obesity; NORM = Normal Weight Controls; BMI = Body Mass Index. Values for age represent years, for weight represent kilograms, for height represent meters. Formula for Body Mass Index is subject's weight multiplied by .45 in the numerator divided by subject's height multiplied by .025 in the denominator. Values in parentheses represent standard deviations. * $\mathrm{p}<.00001$, degrees of freedom for all ANOVAs were (4, 142). 


\section{Assessment Measures}

Several assessment instruments were administered to both clinical and nonclinical subjects. All subjects were interviewed with the Interview for the Diagnosis of Eating Disorders-IV (IDED-IV), and administered the Eating Attitudes Test (EAT: Garner \& Garfinkel, 1979), the Bulimia Test-Revised (BULIT-R: Thelen, Farmer, Wondeerlich, \& Smith, 1991), the Eating Disorder Inventory-II (EDI-II: Garner et al., 1990), and the Body Image Assessment procedure (BIA: Williamson et al., 1990). The IDED-IV was described in a previous section; therefore, only the selfreport measures will be described below.

EAT. The EAT (Garner \& Garfinkel, 1979) is a 40-item self-rating scale which assesses anorexic attitudes regarding eating and weight (see Appendix $C$ ). The patient is asked to rate the frequency of a variety of symptoms typically associated with anorexia. A factor analysis conducted with the EAT identified three factors including Dieting, Bulimia and Food Preoccupation, and oral Control (Garner et al., 1982). Reliability for the test was reported to be $r=.79$ for a clinical sample of anorexics, and $r=.94$ for a sample of anorexics and normal subjects. A positive correlation $(r=.87)$ between the total EAT score and anorexic versus normal group membership was supportive of concurrent validity (Garner \& Garfinkel, 1979). The EAT has also been found to discriminate between 
a bulimic and control sample (Gross, Rosen, Leitenberg, \& Willmuth, 1986). Williamson (1990) reported that both anorexics and bulimics score very high on the EAT. Compulsive overeaters generally have scores between that of anorexics and bulimics and that of obese patients. Thus, the EAT discriminates bulimia nervosa from normals, and anorexia nervosa from normals, but no study has shown that it discriminates anorexia nervosa and bulimia nervosa. Thus, it is best used as general index of anorexic characteristics, especially those concerned with fear of weight gain, drive for thinness, and restrictive eating patterns.

BULIT-R. The BULIT-R (Thelen et al., 1991) is 28-item measure of DSM-III-R (APA, 1987) criteria of bulimia nervosa (see Appendix D). The BULIT-R discriminates bulimia (binge eating) from bulimia nervosa, based on cut-off scores. The BULIT-R has been found to have a high level of internal consistency (Brelsford, Hummel, \& Barrios, 1992) and temporal stability (Brelsford et al., 1992; Thelen et al., 1991). Support for the concurrent validity, using the correlation of BULIT-R scores with group membership (bulimia versus normals) was found to be high, $r=.74$ (Thelen et al., 1991). The validity of the individual items was established by correlating item scores with group membership. For the 28 scored items, the point biserial correlation ranged from .39 to .79 ( $p<.001$; mean $r$ of 
items $=.58)$. Construct validity has been reported for the correspondence between BULIT-R scores and self-monitored frequency of bulimic symptoms (Brelsford et al., 1992) and the BULIT-R and two other measures of bulimic behavior (Thelen et al., 1991). Thelen et al. (1991) conducted a factor analysis of the BULIT-R, using all 28 items. Five factors were derived including (1) binging, (2) radical weight loss measures, (3) laxative/diuretic abuse, (4) vomiting, and (5) exercise.

EDI-II. The EDI-II (Garner et al., 1990) is a 91 item measure designed to measure cognitive and behavioral dimensions that may discriminate subgroups of eatingdisorder patients from normal dieters. The EDI-II is typically used in the assessment of anorexia nervosa and bulimia nervosa. No comparisons of binge eaters or nonbinging obese with anorexia nervosa and bulimia nervosa have been made. Eleven constructs are measured by the EDI: (1) drive for thinness, (2) bulimia (i.e., binge eating); (3) body dissatisfaction, (4) ineffectiveness, (5) perfectionism, (6) interpersonal distrust, (7) interoceptive awareness, (8) maturity fears, (9) asceticism, (10) impulse regulation, and (11) social insecurity. The internal consistency of 9 of the 11 subscales was found to be above .80 (exceptions Asceticism alpha $=.70$ and impulse regulation alpha $=.77$ ). Moderate to high test-retest reliability has been reported for the 
majority of the subscales $(r=.41$ to .97$)$. Strong support was found for the convergent and discriminant validity of each subscale using a variety of self-report measures. The EDI-II items have been found to generally discriminate between eating disorder and normal groups. The EDI-II has also been found to be sensitive to clinical changes. BIA. The BIA (Williamson, Kelley, Davis, Ruggiero, \& Blouin, 1985) is a measure designed to assess a subject's perception of current body size (CBS) and ideal body size (IBS) through the use of body silhouettes which are printed on six-inch by four-inch cards (see Appendix E). One silhouette is printed per card, and there are nine cards. On each card there is a drawing of a female figure whose body size ranges from very thin to very obese, in incremental steps. The cards are placed in random order on a flat surface. The subject is then instructed to "Select the card that most accurately depicts your current body size, as you perceive it to be. Please be honest. You must choose only one card and you may not rearrange the cards to directly compare them." After the subject selects a card, the number of the card (which is on the back of each card) is recorded, the cards are shuffled, and again placed in random order on the flat surface. The subject is then instructed to "Please select the card that most accurately depicts the body size that you would most prefer. Again, be honest and do not rearrange the cards." The number of the 
card that the subject selects is then recorded. This procedure typically takes less than one minute. From these data, one can derive a subject's perception of her current body size (CBS) as well as her ideal body size (IBS).

\section{Procedure}

Phase II: Reliability study. Informed consent was obtained from subjects prior to their participation in the study (see Appendix B). The entire sample of subjects was utilized in the evaluation of the IDED-IV's internal consistency using coefficient alphas and item-total correlations. The interrater reliability of the IDED-IV was then evaluated with 82 the total 176 subjects. Forty percent (38 of 96 ) of the clinical subjects, and $55 \%$ of the nonclinical subjects (44 of 80 ), who agreed to have their IDED-IV interviews audiotaped, had their interviews rated by a second blind interviewer. Raters made diagnostic decisions based on 15 possible eating disorder diagnoses and two control group options (i.e., Obese and Normal Weight Control). A kappa coefficient was calculated based on the agreement between raters for 17 possible classifications in the current study.

Nine of the 11 interviewers participated in the interrater reliability study. The interviewers included eight pre-doctoral level graduate students in clinical psychology, and three post-doctoral unlicensed psychologists. Pre-doctoral level interviewers had at least 
one year of graduate training in the assessment and treatment of eating disorders, which included intensive training in the administration of earlier editions of the IDED. Post-doctoral level interviewers completed specialized training in the assessment (i.e., which included administration of earlier editions of the IDED) and treatment of eating disorders during their graduate careers, and were working in eating disorders programs for up to two years post-graduation. Seven of the pre-doctoral students were in training at Louisiana State University, and two of the post-doctoral students worked with the eating disorders program in a private hospital in Baton Rouge. One of the pre-doctoral students and one of the post-doctoral level interviewers were conducting research and completing clinical work at the Renfrew Center in Philadelphia, Pennsylvania.

The eight pre-doctoral students and the two postdoctoral interviewers located in Louisiana received intensive training in the administration of the newly revised IDED-IV. The third post-doctoral interviewer in Pennsylvania received detailed written instructions regarding administration of the IDED-IV which was further elaborated upon in a telephone discussion. I conducted an intensive two-hour training session with the pre- and postdoctoral level interviewers in Louisiana. Mock interviews were then conducted with the pre-doctoral interviewers 
which were videotaped and reviewed by me, and discussed during supervision with a licensed clinical psychologist. The post-doctoral interviewer in Pennsylvania conducted a similar training session with the pre-doctoral interviewer participating in data collection, and the results of this training session were reviewed with me.

The training sessions on the administration of the IDED-IV focused on providing interviewers with a systematic method for determining diagnosis. Interviewers were trained to have a tentative diagnosis by the completion of the semi-structured overview in the IDED-IV which focuses on obtaining a history of eating-disorder symptoms and general questions about current functioning. Interviewers were then trained with regard to how the structure of the IDED-IV lends itself to collecting information necessary for interviewers to determine whether an individual has a particular symptom and how to rate the severity of that symptom. Figures 1 through 3 outline the specific set of rules interviewers used to make differential diagnosis among the eating disorders.

The diagnostic sections each begin with a heading which cues the interviewer with regard to the DSM-IV diagnostic criterion being rated at the end of each set of questions within the interview. Ratings were made based on subjects' responses to the questions that precede each diagnostic criterion. A rating of three or more on each of 
the symptoms is the operational definition for concluding that the individual has indicated the presence of a diagnostic symptom. Upon completion of the diagnostic interview, interviewers were referred to a diagnostic checklist at the end of the interview which contained detailed instructions for making DSM-IV eating disorder. diagnoses.

Subjects who were identified as having Anorexia Nervosa were those who had a body weight less than $85 \%$ of that expected, intense fear of gaining weight, a body image disturbance, and amenorrhea. Subjects were then assigned to one of two subtypes within the Anorexia Nervosa diagnostic category. Those subjects who did not engage in binge eating or purging behavior were subtyped, "Restricting Type," while those who did engage in binge eating and purging were subtyped, "Binge-Eating/Purging Type" .

Subjects who were identified as having a Binge-Eating Disorder were those who reported binge eating a large amount of food in a discrete period of time, having a loss of control during binge eating, and having at least three of five indicators of loss of control. The five behavioral indicators of loss of control were eating more rapidly than normal, eating until uncomfortably full, eating large amounts when not hungry, eating alone because of embarrassment, and feeling disgusted with oneself after 
overeating. Subjects also needed to report experiencing marked distress regarding binge eating, and the frequency of binge eating occurring at least two days per week for at least six months. The absence of purging behavior is necessary for both making the Binge-Eating Disorder diagnosis and for differentiating between a diagnosis of Binge-Eating Disorder and Bulimia Nervosa. An additional requirement includes that the binge eating not occur exclusively during the course of Anorexia Nervosa or Bulimia Nervosa.

For subjects to be diagnosed with Bulimia Nervosa, they needed to report recurrent episodes of binge eating and a loss of control during binge eating. They also reported recurrent inappropriate compensatory behavior to prevent weight gain which included self-induced vomiting, the misuse of laxatives and diuretics, fasting, and/or excessive exercise. In addition, they needed to report binge eating and inappropriate compensatory behaviors as occurring at least two days per week for three months. They also reported that their self-evaluation was unduly influenced by body shape and weight. Finally, the syndrome did not occur exclusively during episodes of Anorexia Nervosa. Once the diagnosis of Bulimia Nervosa was established, the specific subtype needed to be identified. "Purging Type" was used to designate individuals who engaged in regular use of self-induced vomiting or laxative 
and/or diuretic abuse to purge themselves of food.

"Nonpurging Type" was used to designate individuals who engaged in fasting or excessive exercise to purge their bodies of food, but did not engage in self-induced vomiting or laxative and/or diuretic abuse.

Subjects who reported an aberrant eating pattern that was causing marked distress, or those who reported subthreshold levels of eating disorder symptoms which did not meet the severity or frequency specified in the diagnostic criteria were diagnosed with Eating Disorder Not Otherwise Specified (ED NOS). In DSM-IV, the ED NOS diagnostic category includes Binge-Eating Disorder and five other types of aberrant eating patterns. The ED NOS category also allows the inclusion of individuals who meet a significant number of eating disorder criteria but fall short of meeting full criteria, and who report marked distress regarding the aberrant eating pattern or have a negative impact on their daily functioning.

There are five types of aberrant eating patterns specified in the Eating Disorder Not otherwise Specified diagnostic category. The first type includes individuals who meet the criteria for Anorexia Nervosa except that they have regular menses. The second type includes individuals who meet full criteria for Anorexia Nervosa, but despite significant weight loss, the individual's weight is in the normal range. The third type is reserved for individuals 
who meet the criteria for Bulimia Nervosa except that binges occur at a frequency of less than twice per week or for less than three months. The fourth type includes individuals who are of normal body weight who regularly engage in purging after eating small amounts of food. The fifth type describes individuals who repeatedly chew and spit out, and do not swallow, large amounts of food. And finally, the sixth type is Binge-Eating Disorder which includes the criteria described above.

Phase III: Validity study. The validity of the diagnoses derived in Phase II using the IDED-IV was evaluated in Phase III of the current study using a subset of the initial sample of subjects (i.e., 147 of the total 176 subjects). Based on subjects' diagnostic profiles derived through administration of the IDED-IV, subjects were assigned to one of five groups as described in a previous section. Following administration of the IDED-IV, each subject was asked to complete the Eating Attitudes Test (EAT), the Bulimia Test-Revised (BULIT-R), the Eating Disorders Inventory-II (EDI-II), and the Body Image Assessment (BIA) in random order across subjects.

In addition, each subject's height and weight was obtained, and their body mass index calculated. Body mass indices for the subjects were calculated by multiplying each subject's weight by .45 , and each subject's height by 
.025 , and dividing the former by the latter. Subjects were all staffed with a licensed clinical psychologist after the assessment procedure was completed. 
Results

Following the development of the IDED-IV in Phase I, its psychometric properties were evaluated in Phases II and III of the current study. Phase II focused on examining the reliability of the interview. Phase III involved evaluating the interview's validity.

Phase II: Reliability of the IDED-IV

Internal consistency. The entire sample of subjects was utilized to evaluate the internal consistency of the IDED-IV Subscales using coefficient alpha and item-total correlations. Coefficient alphas were used to determine the average degree of inter-item consistency (Novick \& Lewis, 1967). Coefficient alpha has been described as being a conservative, lower boundary estimate of a test's reliability (Novick \& Lewis, 1967). Derived alpha values were high for all of the subscales. The values were .75 for the Anorexia Nervosa Subscale, .96 for the Binge-Eating Disorder Subscale, and .75 for the Bulimia Nervosa Subscale.

Item-total correlations were also calculated for the IDED-IV to evaluate the strength of the relationship between each rating and each subscale's total score, and to determine whether some ratings made more significant contributions and thus were more important in deriving IDED-IV Subscale total scores. Table 10 summarizes these 
Table 10

Item-Total Correlations Between IDED-IV Individual Ratings and Subscale Totals

$$
\text { IDED-IV Subscales }
$$

Ratings Anorexia Nervosa Binge-Eating Bulimia Nervosa

IDED-IV AN

$\begin{array}{ll}\text { A } & .52 \\ \text { B } & .75 \\ \text { C1 } & .74 \\ \text { C2 } & .74 \\ \text { C3 } & .61 \\ \text { D } & .57\end{array}$

$\underline{\text { IDED-IV BED }}$

$\begin{array}{llll}\text { A1 } & -- & .94 & - \\ \text { A2 } & -- & .93 & - \\ \text { B1 } & -- & .90 & - \\ \text { B2 } & -- & .92 & - \\ \text { B3 } & -- & .83 & - \\ \text { B4 } & -- & .86 & - \\ \text { B5 } & -- & .94 & -- \\ \text { C } & -- & .91 & -\end{array}$


IDED-IV Subscales

Ratings Anorexia Nervosa Binge-Eating Bulimia Nervosa

IDED-IV BN

$\begin{array}{lccc}\text { A1 } & -- & -- & .62 \\ \text { A2 } & -- & -- & .69 \\ \text { B1 } & -- & -- & .58 \\ \text { B2 } & -- & -- & .55 \\ \text { B3 } & -- & -- & .54 \\ \text { B4 } & -- & -- & .50 \\ \text { D } & -- & -- & .76\end{array}$

Note. IDED-IV AN = Interview for the Diagnosis of Eating Disorders-Fourth Revision Anorexia Nervosa Subscale Ratings: $A=15 \%$ below average body weight, $B=$ fear of weight gain, $\mathrm{Cl}=$ feels fat even if not overweight, $\mathrm{C2}=$ influence of body image on self-evaluation, $\mathrm{C} 3=$ denial of seriousness of low body weight, and $D=$ amenorrhea. IDEDIV $B E D=$ IDED-IV Binge Eating Disorder Subscale Ratings: AI $=$ recurrent binge eating, $\mathrm{A} 2=$ loss of control of binge eating, $\mathrm{B} 1$ = rapid eating, $\mathrm{B} 2$ = eating until uncomfortably full, B3 = eating when not hungry, B4 = eating alone due to embarassment, $\mathrm{B} 5=$ negative affect post-binge, $\mathrm{C}=$ marked distress regarding binge eating. IDED-IV BN = IDED-IV Bulimia Nervosa subscale Ratings: $\mathrm{Al}=$ recurrent binge eating, $\mathrm{A} 2=$ loss of control during binge eating, $\mathrm{BI}=$ vomiting, $\mathrm{B} 2$ = laxative/diuretic abuse, $\mathrm{B} 3=$ fasting, $\mathrm{B} 4=$ excessive exercise, $D=$ influence of body image on selfevaluation. 
correlations. Item-total correlations were found to be moderate to high (.52 to .75) for the Anorexia Nervosa Subscale, high to very high $(.83$ to .94) for the BingeEating Disorder Subscale, and moderate to high (.50 to .76) for the Bulimia Nervosa Subscale. Thus, all the ratings across the subscales were found to have at least a moderate relationship with their respective subscale total scores.

Interrater reliability. The utility of the IDED-IV in assisting examiners in making consistent DSM-IV eating disorder diagnoses was evaluated. A kappa coefficient was used to reduce the likelihood of chance agreement. The kappa coefficient was derived based on interrater agreement for 17 possible classifications which described subjects' eating disorder diagnoses and/or weight status. These 17 classifications included the 15 diagnostic categories listed in Table 8 , plus the obese Control and Normal Weight Control groups. Initial interviews were conducted by any 1 of 11 raters, while only 2 of these 11 raters reviewed audiotapes of the initial interviews and arrived at a second diagnosis for selected subjects. The kappa coefficient was found to be .86 which is in the "almost perfect" range of interrater agreement (Landis \& Koch, 1977, p. 165)

Although interrater agreement for subjects' diagnoses would be more practically useful in clinical settings, interrater agreement for the individual ratings was 
examined in order to evaluate whether the IDED-IV ratings and the questions leading up to the ratings were interpreted consistently across the interviewers. Thus, by evaluating interrater agreement across the ratings, the clarity of the descriptors within the ratings and the room left for raters' subjective judgements was evaluated. Interrater agreement was evaluated using percent agreement for the presence or absence of particular eating disorder symptoms, percent agreement for the severity of the symptom being evaluated by a particular rating, and spearman's rank order correlation coefficients. Interrater agreement for the presence or absence of eating disorder symptoms was of particular importance given that these decisions were essential for arriving at DSM-IV eating disorder diagnoses. Table 11 summarizes the percent agreement between interviewers for making this distinction across the three IDED-IV Subscales. Across all three subscales, interviewers had high rates of agreement (i.e., $87.6 \%$ to $98.7 \%$ ) regarding the presence or absence of eating disorder symptoms with one exception. On the Anorexia Nervosa Subscale, interviewers were found to have a moderate level of agreement (i.e., 64.2\%) with regard to the fear of weight gain. Nevertheless, interviewers had a high level of consistency in making eating disorder diagnoses. 
Percent Agreement for Ratings Between Interviewers to Indicate Presence or Absence of Eating Disorder Symptoms

\section{IDED-IV Ratings Percent Agreement}

Anorexia Nervosa Subscale

$\begin{array}{ll}\text { A } & 97.5 \\ \text { B } & 64.2 \\ \text { C1 } & 87.7 \\ \text { C2 } & 92.6 \\ \text { C3 } & 97.5 \\ \text { D } & 93.8\end{array}$

Binge Eating Disorder Subscale

$\begin{array}{ll}\text { A1 } & 88.9 \\ \text { A2 } & 97.6 \\ \text { B1 } & 90.1 \\ \text { B2 } & 91.4 \\ \text { B3 } & 95.1 \\ \text { B4 } & 92.6 \\ \text { B5 } & 92.6 \\ \text { C } & 87.6\end{array}$


Bulimia Nervosa Subscale
A1
88.9
A2
97.6
BI
98.7
B2
95.0
B3
90.1
B4
96.3
D
93.8

Note. IDED-IV = Interview for the Diagnosis of Eating Disorders-Fourth Revision. Anorexia Nervosa Subscale Ratings: $A=15 \%$ below average body weight, $B=$ fear of weight gain, $\mathrm{C1}=$ feels fat even if not overweight, $\mathrm{C2}=$ influence of body image on self-evaluation, $\mathrm{C3}=$ denial of seriousness of low body weight, and $D=$ amenorrhea. Binge Eating Disorder Subscale Ratings: $A 1=$ recurrent binge eating, $\mathrm{A} 2=$ loss of control of binge eating, $\mathrm{B} 1=$ rapid eating, $\mathrm{B} 2$ = eating until uncomfortably full, $\mathrm{B} 3$ = eating when not hungry, $\mathrm{B} 4=$ eating alone due to embarassment, B5 = negative affect post-binge, $\mathrm{C}=$ marked distress regarding binge eating. IDED-IV Bulimia Nervosa Subscale Ratings: A1 $=$ recurrent binge eating, $A 2=$ loss of control during binge eating, $\mathrm{B} 1=$ vomiting, $\mathrm{B} 2$ = laxative/diuretic abuse, $\mathrm{B} 3=$ fasting, $\mathrm{B} 4$ = excessive exercise, $\mathrm{D}=$ influence of body image on self-evaluation. 
Interrater reliability for symptom severity was also evaluated by comparing rates of agreement for the ratings which ranged on a scale of one to five corresponding to level of severity (i.e., one being lowest severity and five being highest severity). A score of one or two on the ratings was indicative of the absence of a particular eating disorder symptom and/or an incidence that was within the normal range. A score of three or higher on the ratings was indicative of subjects having met or having exceeded the threshold for a particular DSM-IV criterion. Table 12 summarizes the rates of interrater reliability and percent agreement for the ratings across the three subscales of the IDED-IV.

Spearman's rank order correlation coefficients and percent agreement were used to evaluate interrater reliability across the individual ratings (see Table 12). Correlation coefficients were low to high $(.41$ to .81$)$ on the Anorexia Nervosa-Subscale, were high (.81 to .90) on the Binge-Eating Disorder Subscale, and moderate to very high (.59 to .90) on the Bulimia Nervosa Subscale. Percent agreement was adequate to high (38.3 to $92.6 \%)$ on the Anorexia Nervosa Subscale, high (70.3 to $85.3 \%$ ) on the Binge-Eating Disorder Subscale, and moderate to high (59.2 to $93.8 \%$ ) on the Bulimia Nervosa Subscale. Thus, moderate to high levels of interrater reliability were found across the IDED-IV ratings except for the "fear of weight gain" 
Comparison of Spearman's Rank-order Correlation

Coefficients and Percent Agreement for IDED-IV Ratings Made by Two Independent Raters
IDED-IV Ratings
$\underline{\underline{r}}_{\mathbf{s}}$
$\%$ Agreement

Anorexia Nervosa Subscale

$\begin{array}{lcc}\text { A } & .52 & 86.4 \\ \text { B } & .58 & 38.3 \\ \text { C1 } & .41 & 64.1 \\ \text { C2 } & .81 & 72.8 \\ \text { C3 } & .54 & 91.3 \\ \text { D } & .68 & 92.6\end{array}$

Binge Eating Disorder Subscale

$\begin{array}{lll}\mathrm{A} 1 & .86 & 70.3 \\ \mathrm{~A} 2 & .90 & 72.7 \\ \mathrm{~B} 1 & .81 & 80.2 \\ \mathrm{~B} 2 & .90 & 80.2 \\ \mathrm{~B} 3 & .86 & 75.2 \\ \mathrm{~B} 4 & .81 & 85.3 \\ \mathrm{~B} 5 & .85 & 76.5 \\ \mathrm{C} & .85 & 74.0\end{array}$

(table con'd) 
IDED-IV Ratings

$\underline{r}_{\mathbf{s}}$

$\%$ Agreement

Bulimia Nervosa Subscale

$\begin{array}{lll}\text { A1 } & .86 & 70.3 \\ \text { A2 } & .90 & 72.7 \\ \text { B1 } & .63 & 93.8 \\ \text { B2 } & .89 & 92.5 \\ \text { B3 } & .66 & 59.2 \\ \text { B4 } & .59 & 81.5 \\ \text { D } & .80 & 69.1\end{array}$

Note. IDED-IV = Interview for the Diagnosis of Eating Disorders-Fourth Revision. Anorexia Nervosa Subscale Ratings: $A=15 \%$ below average body weight, $B=$ fear of weight gain, $\mathrm{Cl}=$ feels fat even if not overweight, $\mathrm{C2}=$ influence of body image on self-evaluation, $\mathrm{C3}=$ denial of seriousness of low body weight, and $D=$ amenorrhea. Binge Eating Disorder Subscale Ratings: $A 1$ = recurrent binge eating, $\mathrm{A} 2=$ loss of control of binge eating, $\mathrm{B} 1=\mathrm{rapid}$ eating, $\mathrm{B} 2$ = eating until uncomfortably full, $\mathrm{B} 3$ = eating when not hungry, B4 = eating alone due to embarassment, B5 $=$ negative affect post-binge, $C=$ marked distress regarding binge eating. IDED-IV Bulimia Nervosa Subscale Ratings: A1 $=$ recurrent binge eating, $A 2=$ loss of control during binge eating, $\mathrm{B} 1=$ vomiting, $\mathrm{B} 2$ = laxative/diuretic abuse, $\mathrm{B} 3=$ fasting, $B 4=$ excessive exercise, $D=$ influence of body image on self-evaluation. 
(Rating B), and the "feels fat even if not significantly overweight" (Rating C1) criteria on the Anorexia Nervosa Subscale for which adequate levels of reliability were found. Thus, the IDED-IV was found to be more precise as a diagnostic measure, and less precise as a measure of symptom severity. Phase III: Validity of the IDED-IV

Convergent and discriminant validity. The convergent and discriminant validity of the IDED-IV Subscales was first evaluated using Pearson correlation coefficients which examined the relationships both within the subscales and between the subscales and self-report measures of related and unrelated constructs. Self-report measures used in the comparisons were selected based on their correspondence to DSM-IV eating disorder criteria. Thus, factors on the Eating Attitudes Test (EAT) and the Bulimia Test-Revised (BULIT-R), and subscales on the Eating Disorders Inventory-II (EDI-II) and the Body Image Assessment (BIA) were included that measured dieting motivation and behavior, body image disturbance, binge eating, and compensatory behavior (e.g., purging). Factors and subscales that were excluded were those that reflected redundant content across measures, as well as those that reflected associated features of eating disorders rather than primary diagnostic criteria. 
Several predictions were made with regard to the nature of the correlations between the IDED-IV Subscales and the dependent measures as reviewed below. Table 13 summarizes the intercorrelations within the IDED-J.V Subscales. Table 14 summarizes the intercorrelations between the IDED-IV subscales and the dependent measures.

Consistent with the predicted intercorrelations between the IDED-IV Subscales, the Anorexia Nervosa and Bulimia Nervosa Subscales were found to be moderately positively correlated. In addition, the Anorexia Nervosa and Binge-Eating Disorder Subscales were found to have a low positive correlation. Dissimilar to the prediction that a moderate positive correlation would be found between the Bulimia Nervosa and Binge-Eating Disorder Subscales, a high correlation was found.

With regard to the pattern of predicted correlations between the IDED-IV subscale totals and selected selfreport measures of eating disorder symptoms, several predictions were confirmed while some were refuted as summarized in Table 14. Consistent with predictions made, the IDED-IV Anorexia Nervosa Subscale was found to have: a high positive correlation with the EAT Dieting Factor, low to moderate positive correlations with the BULIT-R Factors, and a low negative correlation with the BIA for Ideal Body size. Inconsistent with the predictions made, Anorexia 
Table 13

Intercorrelations between the IDED-IV Subscale Totals

\begin{tabular}{llll}
\hline & \multicolumn{3}{c}{ IDED-IV Subscales } \\
& AN & BED & BN \\
IDED-IV Subscales & & & \\
AN & -- & .22 & $.63 *$ \\
BED & -- & - & $.70 *$ \\
BN & -- & -- & - \\
\hline
\end{tabular}

Note. IDED-IV = Interview for the Diagnosis of Eating Disorders-Fourth Edition, $\mathrm{AN}=$ Anorexia Nervosa, $\mathrm{BED}=$ Binge Eating Disorder, $\mathrm{BN}=$ Bulimia Nervosa. $* \underline{p}<.0001$ 
Table 14

Intercorrelations Between the IDED-IV Subscale Totals and Scores on Self-Report Measures of Eating Disorder Symptoms

$$
\text { IDED-IV Subscales }
$$

Self-Report

AN

BN

BED

Measures

EAT Factors

Dieting

oral Control

BULIT-R Factors

Rad Wt Loss Meas

Vomiting

Lax/Diur Abuse

EDI-II Subscales

Drive for Thin

Body Dissatis
Exercise

$.60 *$

.14

$.68 *$

$.76 *$

$.68 *$

$.44 *$

$.30 *$

$.33 *$

$.48 *$

$.45 *$

$.31 *$

$.29 *$

$.42 *$

BIA Subscales

Current Body Size .12

$.39 *$

$.51 *$

Ideal Body Size -.23

Note. IDED-IV = Interview for the Diagnosis of Eating Disorders-Fourth Edition, $A N=$ Anorexia Nervosa, $B N=$ Bulimia Nervosa, $\mathrm{BED}=$ Binge Eating Disorder, $\mathrm{EAT}=$ Eating Attitudes Test, BULIT-R = Bulimia Test-Revised, EDI-II Eating Disorder Inventory-Second Edition, BIA = Body Image Assessment. Rad $=$ Radical; $w t=$ Weight; Meas = Measures; 
Lax = Laxative; Diur = Diuretic; Thin = Thinness; Dissatis $=$ Dissatisfaction. All correlations greater than .27 are significantly greater than. $* \mathrm{p}<.0001$. 
Nervosa subjects were found to have: moderate rather than high positive correlations on the EAT oral control Factor and the EDI-II Drive for Thinness Subscale, and low rather than moderate positive correlations on the EDI Body Dissatisfaction and BIA for Current Body Size. Nevertheless, the convergent validity of the IDED-IV Anorexia Nervosa Subscale was supported by the moderate to high correlations found on measures of dieting behavior, dieting motivation, and oral control, and by the inverse correlation on a measure of ideal body size. Discriminant validity was supported by the low correlations found between the IDED-IV Anorexia Nervosa Subscale and measures of binge eating and purging.

The convergent and discriminant validity of the IDEDIV Bulimia Nervosa Subscale was evaluated in a similar manner. Consistent with predictions, the IDED-IV Bulimia Nervosa Subscale was found to have: a moderate positive correlation on the EAT Dieting Factor, moderate to high correlations on the BULIT-R Factors, and a low negative correlation on the BIA for Ideal Body size. Contrary to predictions that were made, the IDED-IV Bulimia Nervosa Subscale was found to have: moderate rather than high positive correlations on the EDI-II Drive for Thinness Subscale and the BIA for current Body Size; low rather than moderate or high positive correlations on the EAT Oral Control Factor and the EDI-II Body Dissatisfaction 
Subscale. Thus, the convergent validity of the Bulimia Nervosa Subscale was supported by the moderate to high correlations found on measures of binge eating, compensatory behavior, and dieting. The: convergent validity was also supported by the inverse correlation on a measure of ideal body size. The discriminant validjty was supported by the low correlation found on a measure of oral control. With regard to the IDED-IV Binge-Eating Disorder Subscale, consistent with predictions the subscale was found to have: a low positive correlation with the EAT Dieting Factor and BULIT-R Laxative/Diuretic Abuse Factor, a moderate positive correlation on the EDI-II Drive for Thinness Subscale, a low negative correlation on the BULITR Exercise Factor, and a moderate negative correlation on the EAT oral Control Factor. Inconsistent with the predictions made for the IDED-IV Binge-Eating Disorder were the: moderate rather than low correlations on the BULIT-R Vomiting and Laxative/Diuretic Abuse Factors, moderate rather than high correlations with the EDI-II Body Dissatisfaction Subscale and the BIA for Current Body size, and low rather than moderate correlation with the BIA for Ideal Body size. Thus, the convergent validity of the IDEDIV Binge-Eating Disorder Subscale was supported by the moderate to high correlations on me isures of binge eating, radical weight loss techniques (i.e. particularly vomiting), and body dissatisfaction. 
Support for the discriminant validity of the IDED-IV Binge-Eating Disorder Subscale was provided by the low correlations found with measures of dieting behavior, laxative/diuretic abuse, exercise, and ideal body size (i.e., due to subjects with this disorder not having a body image disturbance). The discriminant validity of the IDEDIV Binge-Eating Disorder Subscale was challenged by the moderate correlations found on measures of radical weight loss techniques including vomiting and laxative and diuretic abuse. However, given the overlap in symptomatology between the Bulimia Nervosa and Binge-Eating Disorder Subscales it is likely that Bulimics who are likely to have scored highly on the Binge-Eating Disorder Subscale may have contributed to these moderate relationships with compensatory behaviors.

Given the predictions that the IDED-IV Subscales would be intercorrelated with each other as well as with selfreport measures of related constructs, a canonical correlation was used to examine all potential relationships among predictor and criterion variables simultaneously, while controlling for the Type I error rate.

In light of the development of diagnostic subtypes within DSM-IV (i.e., Anorexia Nervosa, Restricting Type or Binge-Eating/Purging Type; Bulimia Nervosa, Purging or Nonpurging Type), it was predicted that subjects might have elevations on more than one subscale of the IDED-IV. Thus, 
a canonical correlation analysis was conducted to determine whether subjects with elevations on more than one subscale of the IDED-IV had comparable elevations on dependent measures of related constructs. Dependent measures were chosen based on their correspondence to DSM-IV criteria as described in a previous section.

As seen in Table 15, three canonical correlations resulted from the analysis which were then evaluated for statistical significance using the method described by Stevens (1992). The first $\left(x^{2}=87.11\right.$, df $\left.=33, p<.001\right)$, second $\left(X^{2}=55.98, d f=20, p<.001\right)$, and third $\left(X^{2}=\right.$ 17.68, $\mathrm{df}=9, \mathrm{p}<.05)$ canonical correlations were all found to be statistically significant. However, Hair, Anderson, \& Tatham (1987) caution against using statistical significance as the only means of interpreting canonical relationships, and recommend consideration of the size of the canonical correlations as well as which findings lead to a better understanding of the research problem being studied. Thus, in the current study, the third canonical correlation was not interpreted due to the large drop from the values found for the second canonical correlation for size of the canonical correlation and in statistical significance. Only two significant canonical correlations 
Significant Canonical Correlations Between Predictor IDEDIV Subscale Totals and Criterion Dependent Measures

\begin{tabular}{cccccc}
\hline & $\begin{array}{c}\text { Canonical } \\
\text { Analysis }\end{array}$ & Canonical & & & \\
& Correlation & Root & $\mathrm{x}^{2}$ & $\mathrm{df}$ & $\mathrm{p}$ \\
1 & .90 & .80 & 87.11 & 33 & .001 \\
2 & .80 & .64 & 55.98 & 20 & .001 \\
3 & .53 & .28 & 17.68 & 9 & .050 \\
\hline
\end{tabular}

Canonical Correlations for IDED-IV Subscales Predictor Canonical

Variate Variables Coefficient

\begin{tabular}{lll}
\hline 1 & IDED-IV Binge Eating Subscale & .99 \\
& IDED-IV Bulimia Nervosa Subscale & .65 \\
& IDED-IV Anorexia Nervosa Subscale & .86 \\
& IDED-IV Bulimia Nervosa Subscale & .74 \\
\hline
\end{tabular}

Canonical Correlations for Criterion Measures

Criterion Variable

Canonical coefficient

\section{First Criterion Variate}

BULIT-R Binge Eating Factor $\quad .97$

Body Image Assessment Current Body Size .53

(table con'd) 
Canonical Correlations for Criterion Measures Criterion Variable Canonical Coefficient

\section{First Criterion Variate (Continued)}

BULIT-R Vomiting Factor .50

EAT Oral Control Factor $\quad-.47$

BULIT-R Radical Weight Loss Measures Factor .45

EDI-II Body Dissatisfaction Subscale .34

BULIT-R Laxative/Diuretic Abuse Factor $\quad .27$

EDI-II Drive for Thinness Subscale .21

Body Image Assessment Ideal Body Size .18

BULIT-R Exercise Factor -.11

EAT Dieting Factor $\quad .03$

Second Criterion Variate

EAT Dieting Factor $\quad .93$

BULIT-R Radical Weight Loss Measures Factor $\quad .79$

$\begin{array}{lll}\text { EAT Oral Control Factor } & .68\end{array}$

BULIT-R Exercise Factor $\quad .59$

BULIT-R Vomiting Factor $\quad .56$

Body Image Assessment Ideal Body Size -.50

EDI-II Drive for Thinness Subscale .44

BULIT-R Laxative/Diuretic Abuse Factor $\quad .34$

(table con'd) 
Canonical Correlations for Criterion Measures

Criterion Variable

Canonical Coefficient

Second Criterion Variate (Continued)

BULIT-R Binge Eating Factor $\quad .16$

Body Image Assessment Current Body Size -.09

EDI-II Body Dissatisfaction Subscale -.08

Note. IDED-IV = Interview for the Diagnosis of Eating Disorders-Fourth Revision; BULIT-R = Bulimia Test-Revised; EAT = Eating Attitudes Test; EDI-II = Eating Disorders Inventory-Second Edition. 
were found due to significant intercorrelations between the IDED-IV Binge-Eating Disorder and Bulimia Nervosa Subscales, and the IDED-IV Anorexia Nervosa and Bulimia Nervosa Subscales.

As a result, only the first two canonical functions were further examined to determine the relative importance of each of original variables in deriving the canonical relationships (Hair et al., 1987). Toward this aim, canonical loadings were examined to evaluate the amount of variance each predictor and criterion variable shared with its respective canonical variate. Canonical loadings greater than or equal to .50 or equal to or less than .18 were interpreted. The .50 cutoff has been identified as being indicative of canonical loadings that are "very significant" toward interpreting their respective canonical variates, while the .18 or lower cutoff has been used for determining nonsignificant relationships (Hair et al., 1987, p. 249).

The first significant canonical correlation was indicative of a .90 correlation between the first set of predictor and criterion variates. The canonical root for this function estimated a shared variance of $80 \%$ between the predictor and criterion functions. Predictor variables that most significantly contributed to the predictor variate were the IDED-IV Binge-Eating Disorder Subscale (.99) and the IDED-IV Bulimia Nervosa Subscale (.65) 
totals. Criterion variables that contributed most significantly to the criterion variate were the BULIT-R Binge-Eating Factor (.97), the Body Image Assessment Procedure for Current Body Size (.56), and the BULIT-R Vomiting Factor (.50). The lowest correlations were found with the BIA for Ideal Body size (.18) and the EAT Dieting Factor (.03). Thus, the IDED-IV Binge-Eating and Bulimia Nervosa Subscales appear to be most highly associated with each other and representative of Bulimia Nervosa, Purging Type. The convergent validity was supported by the high associations found with measures of binge eating, vomiting, and large self-perceived body size, while the discriminant validity was supported by the low associations found with measures of ideal body size and dieting behavior. The second significant canonical correlation was indicative of a .80 correlation between the second set of predictor and criterion variates. The canonical root for this function estimated a shared variance of $64 \%$ between the predictor and criterion functions. Predictor variables that most significantly contributed to the second predictor variate were the IDED-IV Anorexia Nervosa Subscale (.86) and the IDED-IV Bulimia Nervosa Subscale (.74) totals. Criterion variables that contributed most significantly to the second criterion variate were the EAT Dieting Factor (.93), EAT oral Control Factor (.68), BULIT-R Radical Weight Loss Measures Factor (.79), BULIT-R Vomiting Factor 
(.56), BULIT-R Exercise Factor (.58), and the BIA for Ideal Body size (.50). A low correlation were found with the BULIT-R Binge-Eating Factor (.16). Thus, the high association between the IDED-IV Anorexia Nervosa and Bulimia Nervosa Subscales appear to represent Anorexia Nervosa Binge-Eating/Purging Type (i.e., primarily purging). Convergent validity was supported by the high associations found with measures of dieting behavior, oral control, extreme weight loss measures, and a body image disturbance. Discriminant validity was supported by the low association found with a measure of binge eating. The convergent and discriminant validity of the IDEDIV was further evaluated by comparing the performance of diagnostic groups identified by the IDED-IV on dependent measures of related and unrelated constructs, respectively. Diagnostic group status was based on subjects' performance on all three subscales of the IDED-IV and on interviewers' subsequerit decisions based on information collected by the IDED-IV with regard to eating disorder and/or weight status. Dependent measures were chosen based on their correspondence to DSM-IV eating disorder criteria as described in a previous section. Thus, given the overlap in symptomatology across eating disorder groups, it was predicted that subjects with these diagnoses would score similarly on measures of common symptoms, and dissimilarly on measures of differentiating symptoms. 
A MANOVA was used to determine whether there were significant group differences with regard to performance on the dependent measures. A MANOVA was used in order to control for the interdependence among the dependent variables (Haase \& Ellis, 1987) which ranged from -.40 to .82. The MANOVA yielded an overall significant effect for group, $F(44,450)=12.66 \mathrm{p}<.0001$, using Wilk's criterion. Univariate analyses of variance (ANOVAs) and Scheffe post- hoc comparisons of group means were then used to identify group differences on the dependent measures as summarized in Table 16.

Several significant group differences were found on dependent measures assessing dieting behavior and motivation that provided evidence for the convergent and discriminant validity of the IDED-IV. Although many comparisons can be made to explore the convergent and discriminant validity of the current data, key group differences important in making differential diagnoses will be reviewed below. Convergent validity was supported by findings that Anorexic and Bulimic subjects reported similar levels of dieting on the EAT Dieting Factor. In addition, eating disorder subjects collectively reported higher dieting motivation on the EDI Drive for Thinness Subscale than obese and Normal weight control subjects. Discriminant validity was supported by the finding that Bulimic subjects reported significantly more frequent 
Table 16

Analysis of Variance and Scheffe Post-Hoc Comparisons of Group Means for Dependent Measures

\begin{tabular}{lllllll}
\hline \multicolumn{9}{c}{ Groups } \\
Variable & AN & BN & BED & OBES & NORM & \\
& & & & & & \\
\hline
\end{tabular}

Dieting

$\begin{array}{lrrrrrr}\text { EAT DIET } & 23.73^{\mathrm{a}} & 19.33^{\mathrm{a}} & 10.23^{\mathrm{b}} & 5.14^{\mathrm{b}} & 7.21^{\mathrm{b}} & 25.34 * * \\ \text { EAT OC } & 9.86^{\mathrm{a}} & 2.81^{\mathrm{b}} & .64^{\mathrm{b}} & .79^{\mathrm{b}} & 2.28^{\mathrm{b}} & 40.05 * * \\ \text { EDI-II DT } & 9.86^{\mathrm{a}} & 8.19^{\mathrm{a}} & 10.79^{\mathrm{a}} & 5.18^{\mathrm{b}} & 5.24^{\mathrm{b}} & 4.70 *\end{array}$

Body Image

$\begin{array}{lcccccc}\text { EDI-II BD } & 9.91^{\mathrm{a}} & 14.81^{\mathrm{ab}} & 22.16^{\mathrm{b}} & 19.95^{\mathrm{ab}} & 13.24^{\mathrm{ab}} & 9.71 * * \\ \text { BIA CBS } & 50.10^{\mathrm{a}} & 68.41^{\mathrm{b}} & 73.33^{\mathrm{b}} & 74.10^{\mathrm{b}} & 51.03^{\mathrm{a}} & 25.59 * * \\ \text { BIA IBS } & 38.48^{\mathrm{a}} & 45.59^{\mathrm{a}} & 59.94^{\mathrm{bc}} & 65.24^{\mathrm{b}} & 49.97^{\mathrm{ac}} & 16.44 * *\end{array}$

Binge-Eating

BULIT BNG $27.95^{\mathrm{a}} \quad 60.30^{\mathrm{b}} \quad 59.74^{\mathrm{b}} \quad 36.45^{\mathrm{a}} \quad 27.86^{\mathrm{a}} \quad 59.04 * *$

Compensatory Behavior

$\begin{array}{lcccccc}\text { BULIT RWLM } & 40.05^{\mathrm{ab}} & 43.96^{\mathrm{a}} & 36.51^{\mathrm{b}} & 24.77^{\mathrm{c}} & 22.86^{\mathrm{c}} & 27.69 * * \\ \text { BULIT VOM } & 5.24^{\mathrm{a}} & 10.48^{\mathrm{b}} & 5.12^{\mathrm{a}} & 3.38^{\mathrm{a}} & 4.03^{\mathrm{a}} & 28.41 * * \\ \text { BULIT LDA } & 6.33^{\mathrm{ab}} & 7.67^{\mathrm{a}} & 5.74^{\mathrm{ab}} & 5.14^{\mathrm{ab}} & 4.79^{\mathrm{b}} & 4.17 * \\ \text { BULIT EXER } & 4.57^{\mathrm{a}} & 4.44^{\mathrm{ac}} & 2.56^{\mathrm{b}} & 2.55^{\mathrm{b}} & 2.93^{\mathrm{c}} & 8.30 * *\end{array}$

Note. Means with different superscripts differ significantly at $\mathrm{p}<.05 . \mathrm{AN}=$ Anorexia Nervosa; $\mathrm{BN}=$ Bulimia Nervosa; $B E D=$ Binge Eating Disorder; OBES = obesity; NORM = Normal Weight Controls. All variables preceded by EAT on Eating Attitudes Test. EAT DIET = EAT Dieting Factor; EAT OC = Oral Control Factor. All variables preceded by EDI-II on Eating Disorders InventoryII. EDI-II DT = EDI-II Drive forThinness Subscale; EDI-II $\mathrm{BD}=\mathrm{EDI}-\mathrm{II}$ Body Dissatisfaction Subscale. All variables 
preceded by BIA on Body Image Assessment Procedure. BIA CBS = BIA Current Body Size; BIA IBS = BIA Ideal Body Size. All variables preceded by BULIT on Bulimia Test-Revised. BULIT BNG = BULIT-R Binge Eating Factor; BULIT RWLM = BULIT-R Radical Weight Loss Measures Factor; BULIT VOM = BULIT-R Vomiting Factor; BULIT LDA = BULIT-R

Laxative/Diuretic Abuse Factor; BULIT EXER = BULIT-R

Exercise Factor. Degrees of freedom for all ANOVAs were $(4,137)$.

$* \underline{\mathrm{p}}<.001 . * * \mathrm{p}<.0001$. 
dieting than Binge-Eating Disorder subjects on the EAT

Dieting Factor. In addition, Anorexic subjects reported ahigher level of oral control than Bulimic subjects on the EAT Oral Control Factor which is consistent with Bulimic individuals experiencing a loss of control during binge eating. Moreover, Bulimia Nervosa and Binge-Eating Disorder subjects reported similar levels of oral control on the EAT oral Control Factor. Finally, Binge-Eating Disorder subjects reported higher levels of dieting motivation than obese control subjects on the EDI-II Drive for Thinness Subscale.

on measures assessing body image disturbance, the convergent and discriminant validity of the IDED-IV was supported. On the BIA for Current Body Size, Bulimic subjects viewed themselves as being significantly overweight, while Anorexic subjects viewed themselves as being average weight despite their significantly below average body weight. Discriminant validity was supported by subjects' differential reports of ideal body weights as measured by the BIA for Ideal Body Size. Bulimic subjects reported an ideal body size that was slightly below average weight, while Binge-Eating Disorder subjects reported an ideal body size that was slightly above average weight. In addition, obese control subjects identified an ideal body size that was significantly overweight. In addition, BingeEating Disorder subjects reported the highest level of body 
dissatisfaction, while Anorexic subjects interestingly reported the lowest level of body dissatisfaction on the EDI-II Body Dissatisfaction Subscale.

The convergent validity of the IDED-IV was supported by findings that subjects with Bulimia Nervosa and BingeEating Disorder reported comparable levels of binge eating on the BULIT-R Binge-Eating Factor. In addition, obese Control subjects reported levels of binge eating comparable to those reported by Normal Weight Control subjects. Discriminant validity was supported by findings that Bulimic subjects reported significantly higher levels of binge eating than Anorexic subjects on the BULIT-R BingeEating Factor. In addition, Binge-Eating Disorder subjects reported higher levels of binge eating than obese control subjects.

On measures of extreme weight loss methods (i.e., compensatory behavior), convergent validity was supported by findings that subjects with Anorexia Nervosa and Bulimia Nervosa had similar means on the BULIT-R Radical Weight Loss Measures Factor. The convergent validity was further supported by the comparable levels of exercise reported for the Binge-Eating Disorder and obese Control subjects. Discriminant validity was supported by findings that Anorexic and Bulimic subjects obtained significantly higher scores on the BULIT-R Radical Weight Loss Measures Factor than those obtained by Binge-Eating Disorder subjects. In 
addition, Binge-Eating Disorder subjects reported using more radical weight loss techniques than obese control subjects. With regard to frequency of vomiting, Bulimic subjects reported significantly higher levels than both Anorexic and Binge-Eating Disorder subjects on the BULIT-R Vomiting Factor. Bulimic subjects also reported the highest levels of laxative and diuretic abuse on the BULIT-R Laxative/Diuretic Abuse Factor, although this level was not significantly different from the rates reported by Anorexic, Binge-Eating Disorder, and Obese Control subjects. With regard to exercise, Anorexic and Bulimic subjects reported comparable levels on the BULIT-R Exercise Factor, which were significantly higher than the comparable levels reported for Binge-Eating Disorder and obese Control subjects. In addition, obese control subjects reported lower levels of exercise than Normal Weight Control subjects.

Thus, support was found for several of the predictions made regarding eating disorder subjects' performance on the dependent measures. Anorexic and Bulimic subjects were found to have comparable scores on measures of dieting behavior and motivation, and on measures evaluating a body image disturbance. In addition, Anorexic and Bulimic subjects were found to have dissimilar scores on measures of binge eating and vomiting. However, both groups of subjects were found to have comparable scores on measures 
of radical weight loss techniques including laxative/diuretic abuse and exercise which is likely due to the development of the Anorexia Nervosa BingeEating/Purging Subtype in DSM-IV.

Consistent with predictions made for the Bulimia Nervosa and Binge-Eating Disorder groups, similar levels of binge eating were found for the groups. Also consistent with predictions were the dissimilar levels of dieting behavior, body image disturbance, and compensatory behavior. Thus, the IDED-IV was useful for differentiating between the Bulimia Nervosa and Binge-Eating Disorder groups as supported by dependent measures of related constructs.

Finally, for the Anorexia Nervosa and Binge-Eating Disorder groups, it was predicted that subjects would score dissimilarly on measures assessing dieting behavior, body image disturbance, binge eating, and compensatory behaviors. These predictions were confirmed except that Binge-Eating Disorder subjects were found to have an incidence of vomiting that was not only comparable to that reported for Anorexic subjects, but also to the levels reported for Obese and Normal Weight Control subjects. 
Discussion

The expansion of the eating disorder criteria in the fourth revision of the Diagnostic and statistical Manual (DSM-IV) creates the need for the development of a semistructured interview that would enable differential diagnosis among these diagnostic categories. The Interview for the Diagnosis of Eating Disorders-IV (IDED-IV) was developed to serve this purpose. The purpose of the current study was to describe the development of the IDED-IV, and to evaluate its psychometric properties. Toward these ends, this study was conducted in three phases. Phase I focused on the development of the IDED-IV, Phase II on the evaluation of the interview's reliability, and Phase III on the establishment of the interview's validity. Phase I: Development of the IDED-IV

The development of the IDED-IV involved the application of the DSM-IV eating disorder criteria and the revision of the content and format of an earlier version of the IDED (i.e., IDED-III). The methodology used to develop the IDED-IV was similar to that reported for existing comprehensive diagnostic interviews developed to correspond to diagnostic systems that have evolved over time (i.e., RDI : Helzer et al., 1981; SADS: Endicott \& Spitzer, 1978; SCID: Spitzer et al., 1992; DIS: Robins et al., 1981). The IDED-IV was designed to be similar in structure to a diagnostic clinical interview. The interview begins with a 
semi-structured overview that focuses on basic demographic information, history of eating disorder symptoms, description of chief complaint, and general questions about current functioning. Following completion of the overview, interviewers are guided through fully-structured diagnostic sections that systematically inquire about specific eating disorder symptoms. The structure of the IDED-IV is intended to provide interviewers with a tentative diagnosis by the completion of the overview, and the opportunity to collect information necessary for differential diagnosis in the diagnostic subsections that follow. Upon administration of the interview, interviewers are provided with a diagnostic checklist which both summarizes their observations and provides guidelines for making differential diagnoses. Phase II: Reliability of the IDED-IV

The internal consistency of the IDED-IV Subscales was evaluated using the entire sample of subjects. High levels of internal consistency were found for all three subscales (i.e., Anorexia Nervosa, Binge-Eating Disorder, and Bulimia Nervosa), at values comparable to those reported for established interviews (e.g., SADS: Endicott \& Spitzer, 1978; EDE: Cooper et al., 1989). The interrater reliability of the IDED-IV was evaluated using the kappa coefficient with a subset of the initial pool of subjects. The kappa coefficient was derived based on interrater agreement for 17 possible classifications which described subjects' 
eating disorder diagnoses and/or weight status. The kappa coefficient was found to be .86 which is in the "almost perfect" range of interrater agreement (Landis \& Koch, 1977, p. 165). This kappa value was significantly higher than the kappa values (.37 to .68) reported for comprehensive diagnostic interviews that cover a range of clinical disorders (RDI: Helzer et al., 1981; DIS: Robins et al., 1981; SCID: Spitzer et al., 1992). The lower kappas found for the comprehensive diagnostic interviews may be due to the more numerous options available when making differential diagnoses. Although the CEDRI and the EDE are structured interviews that assess eating disorder symptoms, only the EDE has been adapted to provide DSM-IV diagnoses. However, no studies of the interrater reliability of diagnoses derived through administration of the EDE are available.

Although interrater agreement for subjects' diagnoses would be more practically useful in clinical settings, interrater agreement for the individual ratings was examined in order to evaluate whether the IDED-IV ratings and the questions leading up to the ratings were interpreted consistently across interviewers. Interrater agreement was evaluated using percent agreement for the presence or absence of eating disorder symptoms, percent agreement for the severity of the symptom being evaluated by a particular rating, and spearman's rank order 
correlation coefficients for rating symptom severity. Interrater agreement for the presence or absence of eating disorder symptoms was of particular importance given that these decisions were essential for arriving at DSM-IV eating disorder diagnoses. Across the IDED-IV Subscales, interviewers were found to have high rates of agreement for determining the presence or absence of eating disorder symptoms, with the exception of the "fear of weight gain" criterion on the Anorexia Nervosa Subscale for which a moderate level of agreement was found.

Interrater agreement for symptom severity was also evaluated by comparing rates of agreement within the ratings which ranged on a scale of one to five corresponding to the level of symptom severity (i.e., one being the lowest severity and five being the highest severity). Spearman's rank order correlation coefficients and percent agreement were used to evaluate interrater reliability for symptom severity as measured by the IDED-IV ratings. For nearly all of the IDED-IV ratings, Spearman's rank order correlation coefficients and percent agreement were found to be in the moderate to high range. However, only adequate levels of reliability were found for two criteria on the Anorexia Nervosa Subscale. Adequate levels of reliability were found for percent agreement for "fear of weight gain" (i.e., Rating B), and for the spearman rank order correlation coefficient for "feeling fat even if not 
significantly overweight" (i.e., Rating $C 1$ ). Interrater reliability for the individual ratings on the CEDRI were .59 or greater (Palmer et al., 1987) and for the EDE were .69 or greater (Cooper \& Fairburn, 1987; Wilson \& Smith, 1989). Thus, the interrater reliability of the IDED-IV individual ratings is somewhat below those reported for the CEDRI and EDE. Therefore, the IDED-IV may be more precise as a diagnostic measure and less precise as a measure of symptom severity than the CEDRI and the EDE.

On the IDED-IV Anorexia Nervosa Subscale, the lower rates of agreement for Ratings $B$ and $C 1$ may have been the result of subtle differences among the alternatives provided for describing problem behavior that may have been unclear and left room for interviewers' subjective interpretations. With regard to the rating for fear of weight gain (Rating $B$ ), available options within the rating included, "minimal fear," "moderate fear," "intense fear," "extreme fear," and "debilitating fear." For Rating C1, "feels fat if not significantly overweight," which is intended to assess a body image disturbance, for overweight and obese subjects who reported "feeling fat," it was unclear whether this statement reflected a body image disturbance or mere body dissatisfaction.

In contrast, on the IDED-IV Binge-Eating Disorder Subscale, raters were found to have a high level of agreement on all of the ratings. The high rates of 
agreement may have been due to these ratings assessing more concrete behaviors (e.g., binge eating alone or with others present) which were more straightforward to assess than more abstract symptoms like fear of weight gain or body image disturbance on the Anorexia Nervosa and Bulimia Nervosa Subscales. Interestingly, de Zwaan et al. (1993) found significant disagreements between findings obtained by subjects' self-report versus via diagnostic interview with regard to binge eating behavior. Disagreement between self-report and the diagnostic interview were due to discordances in three of the diagnostic criteria for BingeEating Disorder loss of control during binge eating, marked distress regarding binge eating, and frequency and duration of binge eating. Inconsistencies between subjects and clinicians with regard to the definition of an overeating episode and behavioral indicators of loss of control did not lead to differences between self-report and observer ratings. The current study did not find the difficulties in definition identified in the former study.

Finally, on the IDED-IV Bulimia Nervosa subscale, raters were found to have high levels of agreement when evaluating binge size, loss of control during binges, laxative/diuretic abuse, and influence of body image on self-evaluation. Moderate levels of agreement were found on ratings measuring the incidence of vomiting (Rating B1), dieting (Rating B3), and exercise (Rating B4). The moderate 
levels of agreement found for the vomiting criterion may have been the result of the overlap within the rating with regard to subjects' frequency of vomiting being, "several times per month," versus "several times per week." Moreover, the moderate level of agreement for interviewers ratings of purging through the use of dieting and exercise may have been the result of these constructs not being more specifically defined within the diagnostic criteria thereby leaving room for interviewers' subjective judgments.

Phase III: Validity of the IDED-IV

The convergent and discriminant validity of the IDEDIV Subscales was first evaluated using Pearson correlation coefficients which examined the relationships between the subscales and self-report measures of related and unrelated constructs. Several predictions were made with regard to the nature of the intercorrelations within the IDED-IV Subscales and between the IDED-IV Subscales and self-report measures of eating disorder symptoms. Consistent with the predicted intercorrelations between the IDED-IV Subscales, a moderate positive correlation was found for the Anorexia Nervosa and Bulimia Nervosa Subscales, and a low positive correlation was found between the Anorexia Nervosa and Binge-Eating Disorder Subscales. Contrary to the prediction that a moderate correlation would be found between the Bulimia Nervosa and Binge-Eating Disorder Subscales, a high correlation was found. The high correlation found between 
the Bulimia Nervosa and Binge-Eating Disorder Subscales is likely to be due to the Binge-Eating Disorder Subscale's almost exclusive focus on binge eating for which Bulimic subjects also obtained high scores.

The convergent and discriminant validity of the IDEDIV Subscales was supported in the relationships found between each of the IDED-IV Subscales and measures of related and unrelated constructs, respectively. The convergent validity of the IDED-IV Anorexia Nervosa Subscale was supported by findings that subjects who obtained high scores on the subscale also obtained high scores on measures of dieting behavior, dieting motivation, and oral control. These findings are consistent with those of previous studies (see Kerr, Skok, \& McLaughlin, 1991 for review). The convergent validity of the Anorexia Nervosa Subscale was further supported by the low scores found on a measure of ideal body size which would be expected given Anorexic subjects' preference for a low body weight. Previous studies have produced inconsistent findings regarding the nature of the body image disturbance in individuals with Anorexia Nervosa (see Cash \& Brown, 1987 for review). However, the preponderance of these studies provide support for the presence of a distorted body image, which is one of the defining criteria for this disorder 
(Kaffman \& Sadeh, 1989; Gardner \& Moncriett, 1988; Halmi, 1987; Anderson \& Hay, 1985; Muuss, 1985; Piazza, Rollins, \& Lewis, 1983).

Moreover, the discriminant validity of the Anorexia Nervosa Subscale was supported by the low correlations found with measures of binge eating and purging. The discriminant validity was challenged by the low correlation found with measures of body dissatisfaction and perceived (i.e., current) body size. Thus, it would appear that there is a great deal of variability in Anorexic subjects' selfreported level of body dissatisfaction and current body size estimates as has been confirmed in a review of studies examining this phenomenon (see Cash \& Brown, 1991 for review). However, this finding may in part be due to Anorexic patients' tendency to minimize body weight and shape concerns when seeking treatment, in an effort to delay or avoid program components aimed at promoting weight gain.

The convergent and discriminant validity of the IDEDIV Bulimia Nervosa Subscale was also supported by the moderate to high correlations found on measures of binge eating, compensatory behavior, dieting behavior and motivation (i.e., drive for thinness), and perceived body size. Other researchers have found that Bulimia Nervosa subjects obtain high scores on measures of binge eating, compensatory behavior, dieting, and perceived body size 
(see Kerr et al., 1991 for review). The convergent validity was further supported by the inverse relationship found with a measure of ideal body size, which is consistent with Bulimic subjects' preference for a low body weight, and similar to findings reported for a preponderance of studies of body image (Andersen \& Hay, 1985; Phelan, 1987; Post \& Crowther, 1985; Thompson, Berg, \& Shatford, 1987). The discriminant validity of the Bulimia Nervosa subscale was supported by the low correlation found on a measure of oral control which is consistent with the findings of previous studies (see Kerr et al., 1991 for review).

The IDED-IV Binge-Eating Disorder Subscale was also found to have high levels of convergent and discriminant validity. Convergent validity of the Binge-Eating Disorder Subscale was supported by the moderate to high correlations with measures of binge eating, drive for thinness (i.e., dieting motivation), and body dissatisfaction. These findings are consistent with those of other researchers who have also found Binge-Eating Disorder subjects to have high levels of binge eating (de Zwaan et al., 1992; Fichter, Quadflieg, \& Brandl, 1993; Marcus \& Wing, 1987; Marcus, Smith, Santelli, \& Kaye, 1992; Williamson et al., 1990), drive for thinness (de Zwaan et al., 1992; de Zwaan et al., 1993), and body dissatisfaction (Marcus et al., 1990; Williamson et al., 1990; Wilson, Nonas, \& Rosenblum, 1993). 
The discriminant validity of the IDED-IV Binge-Eating Disorder Subscale was also supported by the low correlation found with measures of dieting behavior, laxative/diuretic abuse, and ideal body size. The low correlation with ideal body size is likely the result of subjects with this disorder not having a body image disturbance, and therefore having more variability with regard to ideal body size preference. Interestingly, a low inverse relationship was found on a measure of exercise suggesting that Binge-Eating Disorder subjects have low activity levels. The discriminant validity of the IDED-IV Binge-Eating Disorder Subscale was challenged by the moderate correlations found on measures of radical weight loss techniques including vomiting and laxative/diuretic abuse. However, given the overlap in symptomatology between the Bulimia Nervosa and Binge-Eating Disorder Subscales, Bulimic subjects are also likely to have obtained high scores on the Binge-Eating Disorder Subscale, and thus may have contributed to the moderate relationships found between the subscale and measures of purging behavior. With regard to the pattern of predicted correlations between the IDED-IV subscale totals and selected self-report measures of eating disorder symptoms, several predictions were confirmed while some were refuted. Consistent with predictions made, the IDED-IV Anorexia Nervosa Subscale was found to have a high positive correlation with dieting behavior, low to moderate positive 
correlations with measures of binge eating and compensatory behavior, and a low negative correlation with a measure of ideal body size consistent with DSM-IV diagnostic criteria. Inconsistent with the predictions made, Anorexia Nervosa subjects were found to have moderate rather than high positive correlations on measures of oral control and dieting motivation (i.e., drive for thinness), and low rather than moderate positive correlations with measures of body dissatisfaction and current body size.

The moderate correlation with the measure of oral control may be the result of the inclusion of subjects with the Anorexia Nervosa Binge-Eating/Purging type. These subjects reported lower levels of oral control than Anorexics with the Restricting subtype, and thus produced more variability with regard to the nature of oral control in this sample of Anorexic subjects and hence a lower correlation than anticipated. Moreover, the moderate correlation found between the Anorexia Nervosa Subscale and a measure of dieting motivation (i.e., drive for thinness), and the low correlations found with measures of body dissatisfaction and current body size may reflect Anorexic subjects' tendency to underreport symptoms when presenting for treatment in an effort to avoid treatment components aimed at promoting weight gain. 
On the IDED-IV Bulimia Nervosa Subscale, consistent with predictions, the subscale had a moderate positive correlation with dieting behavior, moderate to high correlations with binge eating and compensatory behavior, and a low negative correlation with ideal body size which are all consistent with the DSM-IV criteria for Bulimia Nervosa. Contrary to predictions that were made, the IDEDIV Bulimia Nervosa Subscale was found to have moderate rather than high positive correlations with measures of dieting motivation (i.e., drive for thinness) and current body size, and low rather than moderate or high positive correlations on measures of oral control and body dissatisfaction. The moderate levels of dieting motivation and current body size, and the low correlation with body dissatisfaction, are likely to reflect the higher variability across subjects than predicted on these dimensions. These lower correlations may also reflect Bulimic subjects' tendency to deny or underreport these symptoms when presenting for treatment in an effort to avoid treatment components that might lead to weight gain. The low correlation found on a measure of oral control is consistent with Bulimic subjects' characteristic failed dieting attempts and tendency to engage in binge eating. With regard to the IDED-IV Binge-Eating Disorder Subscale, several findings were consistent with predictions made and with DSM-IV diagnostic criteria. The subscale was 
found to have low positive correlations with dieting behavior and laxative/diuretic abuse, a moderate positive correlation with dieting motivation (i.e., drive for thinness), a low negative correlation with level of exercise, and a moderate negative correlation with oral control. Inconsistent with the predictions made, the IDEDIV Binge-Eating Disorder Subscale was found to have moderate rather than low correlations with vomiting and laxative/diuretic abuse, moderate rather than high correlations with body dissatisfaction and current body size, and a low rather than moderate correlation reported for ideal body size.

The moderate correlations found between the BingeEating Disorder Subscale and measures of purging behavior may be related to the likelihood that Bulimic subjects would also obtain high scores on this subscale. This would consequently inflate the relationship between the subscale and measures of purging behavior. In addition, other researchers have found evidence for purgative behavior (i.e., vomiting and laxative/diuretic abuse) among obese binge eaters, although purging behavior is much less prevalent than that reported for Bulimic subjects (de Zwaan et al., 1992; Marcus \& Wing, 1987). Moreover, Binge-Eating Disorder subjects reported lower levels of body dissatisfaction, lower current body size, and lower ideal body size than predicted. These findings may be the result 
of the variability across subjects on these dimensions in light of the absence of a body image disturbance which might have otherwise contributed to more consistent responding across subjects.

The convergent and discriminant validity of the IDEDIV Subscales was further evaluated by examining whether subjects with elevations on more than one subscale of the IDED-IV had comparable elevations on self-report measures of related and unrelated constructs, respectively. Thus, a canonical correlation was used to examine all potential relationships between the predictor and criterion variables simultaneously. It was predicted that the eating disorder subjects would have elevations on more than one subscale of the IDED-IV due to the development of diagnostic subtypes within DSM-IV (i.e., Anorexia Nervosa, Restricting Type or Binge-Eating/Purging Type; Bulimia Nervosa, Purging or Nonpurging Type).

Consistent with this prediction, subjects who had high scores on both the Binge-Eating Disorder and Bulimia Nervosa Subscales (i.e., consistent with a Bulimia Nervosa Purging Type diagnosis) were found to have high levels of binge eating, vomiting, and current body size. These subjects were also found to have lower scores on measures of ideal body size and dieting behavior. Furthermore, subjects who had high scores on the Anorexia Nervosa and Bulimia Nervosa Subscales (i.e., consistent with an 
Anorexia Nervosa Binge-Eating/Purging Type diagnosis) also had high scores on measures of dieting behavior, radical weight loss measures, oral control, exercise, vomiting, and ideal body size. A low positive correlation was found on a measure of binge eating which would suggest an emphasis on purging behavior within this sample for the Anorexia Nervosa Binge-Eating/Purging subtype, without significant amounts of binge eating.

Given that the subjects in the eating disorder groups identified by the IDED-IV might have elevations on more than one subscale of the IDED-IV, the convergent and discriminant validity of the diagnostic groups identified by the interviewers was subsequently evaluated by comparing subjects' performance on measures of self-reported eating disorder symptoms. Key differences between the groups that would be critical for differential diagnosis were examined in group comparisons. On measures of dieting behavior and motivation, convergent validity was supported by findings that Anorexic and Bulimic subjects reported similar levels of dieting. In addition, consistent with previous studies (Manley, 1989; Vanderheyden \& Boland, 1987) eating disorder subjects collectively reported higher dieting motivation than Obese and Normal Weight Control subjects. Discriminant validity was supported by the finding that Bulimic subjects reported significantly more frequent dieting than Binge-Eating Disorder subjects which is 
consistent with the findings of other researchers (Brody, Walsh, \& Devlin, 1994; Williamson et al., 1990). In addition, Anorexic subjects were found to have a higher level of oral control than Bulimic subjects, which is likely to be the result of Bulimic individuals experiencing a loss of control during binge eating. Moreover, Bulimia Nervosa and Binge-Eating Disorder subjects reported similar levels of oral control, which were comparable to rates reported for Obese and Normal Weight Controls. Other researchers have found support for individuals with BingeEating Disorder reporting a more frequent loss of control during eating than obese control subjects (Goldfein, Walsh, Lachausse, Kissileff, \& Devlin, 1993; Wilson et al., 1993). Finally, with regard to dieting motivation, BingeEating Disorder subjects reported higher levels of dieting motivation (i.e., drive for thinness) than obese control subjects. This finding is comparable to those reported in previous studies (de Zwaan et al., 1992; de Zwaan et al., 1993). However, Binge Eaters have not been found to differ from obese Controls with regard to dieting behavior due to both groups seldom engaging in consistent dieting (Wilson et al., 1993; Williamson et al., 1990).

On measures assessing body image disturbance, the convergent validity of the IDED-IV was supported by the finding that Anorexic subjects viewed themselves as being average weight despite their significantly below average 
body weight. In addition, Bulimic subjects viewed themselves as being significantly overweight despite the majority of the subjects being from low average to average weight. These findings are consistent with the results of previous studies which have found both Anorexia Nervosa (Bell, Kirkpatrick, \& Rinn, 1986; Freeman, Thomas, Solyom, \& Koopman, 1985), and Bulimia Nervosa subjects to overestimate their body size (Freeman et al., 1985), but inconsistent with a finding that Bulimic and Normal Weight Control subjects had no differences in their perceived body sizes (Cooper \& Taylor, 1988). In addition, Binge-Eating Disorder and obese control subjects accurately identified their body sizes as being significantly overweight at comparable levels as has been reported in previous studies (Williamson et al., 1990).

Discriminant validity was supported on measures of body image disturbance by subjects' differential reports of ideal body weights across the diagnostic groups. Anorexic and Bulimic subjects were found to have an ideal body size that was significantly below average weight which is consistent with the results of previous studies (Cooper \& Taylor, 1988; Davis, Williamson, Goreczny, \& Bennett, 1989). In contrast, Binge-Eating Disorder and obese Control subjects reported an ideal body size that was above average weight as has been found in previous studies (Davis et al, 1989; Williamson et al., 1990), and neither of these groups 
have been found to have a body image disturbance (Williamson et al., 1990; Williamson et al., 1985).

with regard to body dissatisfaction, Anorexic subjects reported the lowest level of body dissatisfaction, although reported levels were only significantly lower than the level reported for the Binge-Eating Disorder group. In contrast, previous studies have found Anorexic subjects to be dissatisfied with their body sizes (Kaffman \& Sadeh, 1989; Gardner \& Moncriett, 1988; Halmi, 1987; Humphrey, 1986). In addition, Bulimic subjects have also been found to have a strong dissatisfaction with their body sizes (Freeman et al., 1985; Humphrey, 1986, Mizes, 1988; Willmuth, Leitenberg, Rosen, \& Cado, 1988). The low levels of body dissatisfaction found for Anorexic and Bulimic subjects may reflect their tendency to underreport levels of dieting and body dissatisfaction when presenting for treatment in an effort to avoid treatment components aimed at weight gain.

Moreover, Binge-Eating Disorder and obese Control subjects reported the highest levels of body dissatisfaction, although reported levels were not significantly higher than the levels reported by Bulimic and Normal Weight Control subjects. Consistent with these results, some studies have found no differences in body dissatisfaction between Binge-Eating and Non-Binge-Eating Obese (Wilson et al., 1993; Williamson et al., 1990; Marcus 
et al., 1990) and Binge-Eating Disorder and Bulimic (Marcus et al., 1992) individuals. However, other studies have found Binge-Eating Disorder subjects to have more body dissatisfaction than Non-binging Obese subjects (Wilson et al., 1993; de Zwaan et al., 1992; Fichter et al., 1993). Researchers have proposed that the discrepancies in the findings across studies of body image disturbance may be due to differences in the way body image was measured across studies (Cash \& Brown, 1987; Wilson et.al., 1993). In a study (Wilson et al., 1993) of binge eating and nonbinge eating Obese subjects, no differences were found between groups on the Body Image Assessment procedure that was also used in this study. However, group differences were found on the Body Image Subscale on the Eating Disorder Examination which evaluates the importance of weight and shape on self-evaluation. This discrepancy may reflect differences in ratings of symptom severity that may result when information is obtained by subject self-report versus expert ratings.

The convergent validity of the IDED-IV was further supported by subjects' performance on measures of binge eating. Subjects with Bulimia Nervosa or Binge-Eating Disorder reported large binge sizes and several behavioral indicators of loss of control during binge eating which is similar to the findings by other researchers (Fichter et al. 1993; Marcus et al. 1992; Marcus \& Wing, 1987; 
Williamson et al., 1990). However, other researchers have reported that binge size is larger for Bulimic than BingeEating Disorder subjects (Brody et al., 1994; Goldfein et al., 1993), and that Binge-Eating Disorder subjects may have a less severe disturbance of eating behavior than individuals with Bulimia Nervosa (Goldfein et al., 1993). Moreover, Obese Control subjects reported levels of binge eating comparable to those reported by Normal Weight Control subjects, although in a previous study, obese subjects were found to binge eat at higher levels than Normal Weight Control subjects (Williamson et al., 1990). On measures of binge eating, discriminant validity was supported by findings that Bulimic subjects reported significantiy higher levels of binge eating than Anorexic subjects. In addition, Binge-Eating Disorder subjects reported higher levels of binge eating than obese control Subjects which is similar to the results of previous studies (de Zwaan et al., 1992; Fichter et al., 1993; Goldfein et al., 1993; Williamson et al., 1990; Wilson et al., 1993). Wilson et al. (1993) also found that Binge Eaters had a greater sense and fear of loss of control during eating than obese control subjects which was not confirmed in the current study.

On measures of extreme weight loss methods (i.e., compensatory behavior), convergent validity was supported by findings that Anorexic and Bulimic subjects reported 
comparable levels of radical weight loss techniques as supported by the findings of a previous study (Swain, Shisslak, \& Crago, 1991). The convergent validity was further supported by the low levels of exercise reported by both Binge-Eating Disorder and Obese Control subjects. Discriminant validity was supported by findings that Anorexic and Bulimic subjects reported significantly higher levels of radical weight loss methods than did Binge-Eating Disorder subjects. In addition, Binge-Eating Disorder subjects used more radical weight loss techniques than Obese Control subjects.

With regard to frequency of vomiting, Bulimic subjects reported significantly higher levels than both Anorexic and Binge-Eating Disorder subjects as would be expected by DSMIV diagnostic criteria. Bulimic subjects also reported the highest levels of laxative and diuretic abuse, although this level was not significantly different from the rates reported by Anorexic, Binge-Eating Disorder, and Obese Control subjects perhaps due to the low incidence of laxative/diuretic abuse in the current sample. The finding that Binge-Eating Disorder subjects had some incidence of purging behavior is inconsistent with DSM-IV diagnostic criteria. However, other researchers have also found evidence for purgative behavior (i.e., vomiting and laxative/diuretic abuse) among Obese Binge Eaters, although purging behavior is much less prevalent than that reported 
for Bulimic subjects (de Zwaan et al., 1992; Marcus \& Wing, 1987). With regard to exercise, Anorexic and Bulimic subjects reported comparable levels, which were significantly higher than the levels reported by BingeEating Disorder and obese Control subjects. In addition, Obese Control subjects reported lower levels of exercise than Normal Weight Control subjects.

Thus, support was found for several of the predictions made regarding eating disorder subjects' performance on the dependent measures. Anorexic and Bulimic subjects were found to have comparable scores on measures of dieting behavior and motivation, and on measures evaluating body image. In addition, Anorexic and Bulimic subjects were found to have dissimilar scores on measures of binge eating and vomiting. However, both groups of subjects were found to have comparable scores on measures of radical weight loss techniques including laxative/diuretic abuse and exercise which is likely due to the development of the Anorexia Nervosa Binge-Eating/Purging Subtype in DSM-IV. Consistent with predictions made for the Bulimia Nervosa and Binge-Eating Disorder groups, similar levels of binge eating were found for the groups. Also consistent with predictions were the dissimilar levels of dieting behavior, body image disturbance, and compensatory behavior. Thus, the IDED-IV was useful for differentiating 
between the Bulimia Nervosa and Binge-Eating Disorder groups as supported by dependent measures of related constructs.

Finally, for the Anorexia Nervosa and Binge-Eating Disorder groups, support was found for the prediction that subjects would score dissimilarly on measures assessing dieting behavior, body image disturbance, binge eating, and compensatory behaviors. The only exceptions to these overall patterns were findings that Binge-Eating Disorder subjects had an incidence of vomiting and laxative/diuretic abuse that were comparable to those for Anorexic subjects, as well as to the incidence for obese and Normal Weight Control subjects. In addition, Binge-Eating Disorder subjects' drive for thinness (i.e., dieting motivation) was comparable to that reported for Anorexic and Bulimic subjects.

Thus, the IDED-IV appears to be a reliable and valid assessment instrument for collecting information and for arriving at diagnostic formulations based on DSM-IV eating disorder diagnoses. The IDED-IV is the first diagnostic interview for the eating disorders based on DSM-IV criteria. The use of the IDED-IV by researchers and clinicians may be helpful toward arriving at diagnostic decisions in a timely manner, to reduce cost and risk that subjects would prematurely discontinue participation during otherwise lengthy assessments. In addition, the structured 
nature of the IDED-IV is not only likely to improve the rate of agreement across interviewers, but will also reduce cost through the use of lay interviewers for administration in research studies. The IDED-IV could prove useful to researchers as a tool for obtaining careful diagnostic delineations of subjects' symptoms and characteristics, and for the identification of homogeneous groups of subjects so that results across studies can be compared and contrasted (Wiens, 1990; Rubinson \& Asnis, 1989). The IDED-IV could also prove useful to clinicians for assisting in determination of diagnoses to aid in treatment planning. Revisions in the major diagnostic systems towards more descriptive and symptom-based diagnoses, combined with the proliferation of diagnoses-specific treatment interventions, have created a need for the development of structured interviews that contain a high degree of standardization (Groth-Marnat, 1990; Shea, 1990; Blain \& Barton, 1979). A structured eating disorders interview allows the detailed probing and questioning of subjects to determine whether reported symptoms represent pathologic body image and eating attitudes and behaviors, or symptoms which are subjectively distressing, but widely held in our weight conscious society (Rosen et al., 1990). The complexity of the attitudinal and behavioral features that are specific to eating disorder psychopathology create the need for a structured interview that enables assessment of 
these dimensions (Cooper \& Fairburn, 1987). The IDED-IV enables this type of assessment by including objective definitions for behavioral symptoms (e.g., binge eating, purging, dieting) to improve the likelihood of diagnostic agreement across interviewers. The IDED-IV also enables the calculation of the frequencies and time frames of specific behaviors that are relevant for diagnostic decisions or the assessment of treatment outcome. It includes consideration of historical and developmental factors unlike previous eating disorder interviews (i.e., CEDRI, EDE).

Limitations of the current study include the low sample sizes of Anorexia Nervosa and Bulimia Nervosa subjects which prohibited the comprehensive study of the newly developed diagnostic subtypes in DSM-IV. Moreover, the inclusion of subjects with subclinical eating disorder symptoms may have made the diagnostic groups less distinct and made less clear the differences between groups. Although several studies have found few differences between subjects meeting full criteria versus subclinical levels of eating disorder symptomatology (Wilson et al., 1993; Wilson, 1992; Wilson \& Walsh, 1991). Consequently, differences across groups may have been less salient, and thus not identified as being significant.

Future studies are needed to further refine the individual ratings within the IDED-IV to reduce ambiguity with regard to the behavioral descriptions within some of 
the ratings, and thus promote higher levels of interrater agreement. The results of the current study also made clear the need for further specification of the eating disorder diagnostic criteria within DSM-IV. In particular, to improve interrater reliability across diagnosticians, several modifications to the DSM-IV eating disorder criteria are needed. These include specifying a standard reference for determining weight status for individuals with Anorexia Nervosa, clarifying the definitions for fear of weight gain and body image disturbance, and providing parameters to define excessive exercise and dieting. Furthermore, studies are needed which compare and contrast the newly developed diagnostic subtypes in the DSM-IV. Preliminary studies that have evaluated the subtypes have found support for the distinctions made in DSM-IV (Garner, Garner, \& Rosen, 1993; McCann, Rossiter, King, \& Agras, 1991; willmuth et al., 1988), although other researchers have argued against the distinctions being made in DSM-IV (Walters et al., 1993). These subtypes are likely to be nosologically helpful to clinicians who found DSMIII-R diagnostic criteria too simplistic for capturing the complexity of the symptomatology of many eating disorder subjects. However, these subtypes are also likely to make comparisons across diagnostic groups more difficult given the overlap in the symptomatology of the different 
subtypes, and may be indicative of the need for a newly conceptualized classification system. 


\section{References}

American Psychiatric Association. (1952). Diagnostic and statistical manual (1st ed.). Washington, D. C.: American Psychiatric Association.

American Psychiatric Association (1968). Diagnostic and statistical manual (2nd ed.). Washington, D. C.: American Psychiatric Association.

American Psychiatric Association (1980). Diagnostic and statistical manual (3rd ed.). Washington, D. C.: American Psychiatric Association.

American Psychiatric Association (1987). Diagnostic and statistical manual (3rd ed., revised). Washington, D. C.: American Psychiatric Association.

American Psychiatric Association. (1993). Diagnostic and statistical manual (4th ed., draft criteria). Washington, D. C.: American Psychiatric Association.

Andersen, A. E. \& Hay, T. (1985). Racial and socioeconomic influences in anorexia nervosa and bulimia. International Journal of Eating Disorders, 4 , 479-481.

Ash, P. (1949). The reliability of psychiatric diagnosis. Journal of Abnormal and Social Psychology, 44, 272277 .

Begelman, D. A. (1976). Behavioral classification. In M. Hersen \& A. E. Bellack (Eds.), Behavioral assessment: A practical handbook. New York: Pergamon Press.

Bell, C., Kirkpatrick, S. W., \& Rinn, R. C. (1986) . body image of anorexic, obese, and normal females. Journal of Clinical Psychology, $\underline{42}, 431-439$.

Blain, D. \& Barton, D. (1979). The history of American psychiatry: A teaching and research quide. Washington, DC: American Psychiatric Association.

Brelsford, T. N., Hummel, R. M., \& Barrios, B. A. (1992). The bulimia test-revised: A psychometric investigation. Psychological Assessment, 4, 399-401.

Brody, M. L., Walsh, B. T., \& Devlin, M. J. (1994). Binge eating disorder: Reliability and validity of a new diagnostic category. Journal of consulting and Clinical Psychology, $\underline{62}, 381-386$. 
Bunnell, D. W., Shenkur, I. R., Nussbaum, M. P., Jacobson, M. S. \& Cooper, P. (1990). Subclinical versus formal eating disorders: Differentiating psychological features. International Journal of Eating Disorders, 9, $357-362$.

Cash, T. F., \& Brown, T. A. (1987). Body image in anorexia nervosa and bulimia nervosa. Behavior Modification, 11, 487-521.

Cooper, P. J., \& Taylor, M. J. (1988). Body image disturbance in bulimia nervosa [Second Leeds Psychopathology symposium: The psychopathology of body image (1986, Leeds, England)]. British Journal of Psychiatry, 153(Suppl 2), 32-36.

Cooper, Z., Cooper, P. J., \& Fairburn, C. G. (1989). The validity of the eating disorder examination and its subscales. British Journal of Psychiatry, 154, 807812 .

Cooper, Z. \& Fairburn, C. (1987). The eating disorder examination: A semi-structured interview for the assessment of the specific psychopathology of eating disorders. International Journal of Eating Disorders, $\underline{6}(1), 1-8$.

Davis, C. J., Williamson, D. A., Goreczny, A. J., \& Bennett, S. M. (1989). Journal of Psychopathology and Behavioral Assessment, 11, 61-69.

de Zwaan, M., Mitchell, J. E., Seim, H. C., Specker, S. M., Pyle, R. L., Mussell, M. P., \& Seim, H. C. (1993). Diagnosing binge eating disorder: Level of agreement between self-report and expert-rating. International Journal of Eating Disorders, 14, 289-295.

de Zwaan, M., Nutzinger, D. O., \& Schoenbeck, G. (1992) . Binge eating in overweight women. Comprehensive Psychiatry, 33, 256-261.

Derogatis, L. R., Lipman, R. S. \& Covp, L. (1973). The SCL90: An out-patient psychiatric rating scale.

Psychopharmacology Bulletin, 9, 13-28.

Endicott, J. \& Spitzer, R. I. (1978). A diagnostic interview. Archives of General Psychiatry, 35, 837844 .

Fairburn, C. G. \& Cooper, Z. (1993). The Eating Disorder Examination (12th ed.). In C. G. Fairburn \& G. T. 
Wilson (Eds.), Binge eating: Nature, assessment, and treatment. (pp. 3-14). New York: Guilford Press.

Feighner, J. P., Robins, E., Guze, S, B., Woodruff, R. A., Winokur, G., \& Munoz, R. (1972). Diagnostic criteria for use in psychiatric research. Archives of General Psychiatry, 26, 57-63.

Fichter, M. M., Quadflieg, N., \& Brandl, B. (1993). Recurrent overeating: An empirical comparison of binge-eating disorder, bulimia nervosa, and obesity. International Journal of Eating Disorders, 14, 1-16.

First, M. B., Frances, A., widiger, T. A., Pincus, H. A. , \& Wakefield-Davis, W. (1992). DSM-IV and behavioral assessment. Behavioral Assessment, 14, 297-306.

Freeman, R. J., Thomas, C. D., Solyom, L., \& Koopman, R. F. (1985). Clinical and personality of body size overestimation in anorexia nervosa and bulimia nervosa. International Journal of Eating Disorders, $\underline{4}$, $439-456$.

Freud, A. (1946). The ego and mechanisms of defense. New York: International Universities Press.

Freud, S. (1910). The origins and development of psychoanalysis. American Journal of Psychology, 21.

Freud, S. (1927). The problem of lay-analyses. New York: Bretano Press.

Gardner, R. M., \& Moncriett, C. (1988). Body image distortion in anorexics as a non-sensory phenomenon: A signal detection approach. Journal of Clinical Psychology, 42, 101-107.

Garner, D. M. (1990). Eating disorder inventory-2: Professional manual. Odessa, FL: Psychological Assessment Resources.

Garner, D. M. \& Garfinkel, P. E. (1979). The eating attitudes test: An index of the symptoms of anorexia nervosa. Psychological Medicine, 9, 273-279.

Garner, D. M. , Garner, M. V. , \& Rosen, L. W. (1993). Anorexia nervosa "restricters" who purge: Implications for subtyping anorexia nervosa. International Journal of Eating Disorders, 13, 171-185.

Garner, D. M., Olmsted, M. P., Bohr, Y., \& Garfinkel, P. E. (1982). Psychological Medicine, 12, 871-878. 
Goldfein, J. A., Walsh, B. T., LaChaussee, J. L., Kissileff, H. R., \& Devlin, M. J. (1993). Eating behavior in binge-eating disorder. International Journal of Eating Disorders, 14, 427-431.

Goodman, W. K., Price, L. H., Rasmussen, S. A., Mazure, C., Fleishmann, R. L., Hill, C. L., Heninger, G. R., \& Charney, D. S. (1989). The Yale-Brown Obsessive Compulsive scale. Archives of General Psychiatry, 46, 1006-1011.

Graham, J. R. (1973). Measuring behavior and adjustment in the community: A factor analytic study of the Katz Adjustment Scale (Form R1). Journal of Community Psychology, 1, 48-53.

Gross, J., Rosen, J. C., Leitenberg, H. , \& Willmuth, M. E. (1986). Validity of the Eating Attitudes Test and the Eating Disorders Inventory in Bulimia Nervosa. Journal of Consulting and Clinical Psychology, 54, 875-876.

Groth-Marnat, G. (1990). The assessment interview. In G. Groth-Marnat (Ed.), Handbook of Psychological Assessment (2nd ed.) (pp. 57-80).

Haase, R. F. \& Ellis, M. V. (1987). Multivariate analysis of variance. Journal of Counseling Psychology, 34, 404-413.

Hair, J. F., Jr., Anderson, R. E., \& Tatham, R. L. (1987). Multivariate data analysis (2nd ed.). New York, NY: Macmillan.

Halmi, K. A. (1987). Anorexia nervosa and bulimia. In V. B. Van Hassell \& M. Hersen (Ed.), Handbook of adolescent psychology (pp. 265-287). New York: Pergamon Press.

Hartmann, H. (1958). Ego psychology and the problem of adaptation. New York: International Universities Press.

Heaton, R. K. , Lehman, R. A. W. , \& Getto, C. J. (1980). Renard diagnostic interview. Archives of General Psychiatry, 38, 393-398.

Helzer, J. E., Robins, L. N. , Croughan, J. L., \& Weiner, A. (1981). Renard diagnostic interview. Archives of General Psychiatry, 38, 393-398.

Herzog, D. B., Hopkins, J. D. , \& Burns, C. D. (1993). A follow-up study of 33 subdiagnostic eating disordered women. International Journal of Eating Disorders, 14, 261-267. 
Humphrey, L. L. (1986). Structural analysis of parent-child relationships in eating disorders. Journal of Abnormal Psychology, 95, 395-402.

Hyler, S. E., Williams, J. B. W. \& Spitzer, R. L. (1982). Reliability in the DSM-III field trials: Interview versus case summary. Archives of General Psychiatry, 39, 1275-1278.

Kaffman, M., \& Sadeh, T. (1989). Anorexia nervosa in a kibbutz: Factors influencing the development of a monoideistic fixation. International Journal of Eating Disorders, $8,33-53$.

Kaplan, H., Freedman, A., \& Sadock, B. (Eds.) (1980). Comprehensive textbook of psychiatry ( $3 \mathrm{rd}$ ed.).

Baltimore: Williams \& Wilkins.

Katz, M. M., Secunda, S. K., \& Hirschenfield, R. M. (1979). NIMH clinical research branch collaborative program on the psychology of depression. Archives of General Psychiatry, 36, 765-771.

Kerr, J. K., Skok, R. L., \& McLaughlin, T. F. (1991) . Characteristics common to females who exhibit anorexic or bulimic behavior: A review of current literature. Journal of Clinical Psychology, 47, 846-853.

Landis, J. R., \& Koch, G. C. (1977). The measurement of observer agreement for categorical data. Biometrics, 33, 159-174.

Lindholm, L., \& Wilson, G. T. (1988). Body image assessment in patients with bulimia nervosa and normal controls. International Journal of Eating Disorders, 7, 527-539.

Manley, R. S. (1989). What are anorexia nervosa and bulimia nervosa? Symposium conducted at the community Health Education Advisory Committee Workshop, Vernon, British Columbia.

Marcus, M. D., Smith, D., Santelli, R., \& Kaye, W. (1992). Characterization of eating disordered behavior in obese binge eaters. International Journal of Eating Disorders, 12, 249-255.

Marcus, M. D., \& Wing, R. R. (1987). Binge eating among the obese. Annals of Behavioral Medicine, 9, 23-27.

Marcus, M. D., Wing, R. R., Ewing, L. D., Kern, E., Gooding,W. \& McDermott, M. (1990). Psychiatric 
disorciers among obese binge eaters. International Journal of Eating Disorders, 9, 69-77.

McCann, U. D., Rossiter, E. M., King, R. J., \& Agras, W. S. (1991). Nonpurging bulimia: A distinct subtype of bulimia nervosa. International Journal of Eating Disorders, 10, 679-687.

Meyer, A. (1951). The collected papers of Adolf Meyer (Vol. 3). E. E. Winters (Ed). Baltimore: John Hopkins Press.

Miller, W. R., \& Marlatt, G. A. (1984). Manual: Comprehensive drinker profile. Odessa, FL: Psychological Assessment Resources.

Mizes, J. S. (1988). Personality characteristics of bulimic and non-eating-disordered female controls: A cognitive behavioral perspective. International Journal of Eating Disorders, 7, 541-550.

Muuss, R. E. (1985). Adolescent eating disorder: Anorexia nervosa. Adolescence, 20, 525-536.

Novick, M. R., \& Lewis, C. (1967). Coefficient alpha and the reliability of composite measurements. Psychometrika, 32, 1-12.

Palmer, R., Christie, M., Cordle, C., Davies, D., \& Kenrick, J. (1987). The clinical eating disorder rating instrument (CEDRI): A preliminary description. International Journal of Eating Disorders, $\underline{6}, 9-16$.

Phelan, P. W. (1987). Cognitive correlates of bulimia: The bulimic thoughts questionnaire. International Journal of Eating Disorders, 56, 593-607.

Piazza, E., Rollins, N., \& Lewis, F. S. (1983). Measuring severity and change in anorexia nervosa. Adolescence, $18,293-305$.

Post, G., \& Crowther, J. H. (1985). Variables that discriminate bulimic from nonbulimic adolescent females. Journal of Youth and Adolescence, 14, 85-98.

Richardson, S. A., Dohrenwend, B. S., \& Klein, D. (1965). Interviewing-Its forms and functions. New York: Basic Books.

Robins, L. N., Helzer, J. E., Croughan, J., \& Ratcliff, K. (1981). National institute of mental health diagnostic interview schedule. Archives of General Psychiatry, $38,381-389$. 
Rosen, J. C., Vara, L., Wendt, S. \& Leitenberg, H. (1990) . Validity studies of the eating disorder examination. International Journal of the Eating Disorders, 9, 519528 .

Rounsaville, B. J., Kosten, T. R., Williams, J. B. W. \& \& Spitzer, R. L. (1987). A field trial of DSM-III-R psychoactive substance dependence disorders. American Journal of Psychiatry, 144, 351-355.

Rubinson, E. P. \& Asnis, G. M. (1989). Use of structured interviews for diagnosis. In $S$. Wetzler (Ed.), Measuring mental illness: Psychometric assessment for clinicians (pp. 43-68). Washington, DC: American Psychiatric Press.

Scarano, G. M., \& Kalodner-Martin, C. R. (1994). A description of the continuum of eating disorders: Implications for intervention and research. Journal of Counseling and Development, $\underline{72}, 356-361$.

Shea, S. C. (1990) . Contemporary psychiatric interviewing: Integration of DSM-III-R, psychodynamic concerns, and mental status. In G. Goldstein \& M. Hersen (Eds.), Handbook of psychological assessment (2nd ed.) (pp. 283-307). New York: Pergamon Press.

Skodol, A., Rosnick, L., Kellman, D., Oldham, J. M., \& Hyler, S. E. (1988). Validating structured DSM-III-R personality disorder assessments with longitudinal data. American Journal of Psychiatry, 145, 1297-1299.

Spitzer, R. G., Endicott, J. \& Robins, E. (1975) . Clinical criteria for diagnosis and DSM-III. American Journal of Psychiatry, 132, 1187-1192.

Spitzer, R. G., Endicott, J. \& Robins, E. (1978). Research diagnostic criteria rationale and reliability. Archives of General Psychiatry, 35, 773-782.

Spitzer, R. L., \& Fleiss, J. L. (1974). A reanalysis of the reliability of psychiatric diagnosis. British Journal of Psychiatry, 125, 341-347.

Spitzer, R. L., Forman, J. B. \& Nee, J. (1979). DSM-III field trials: Initial interrater diagnostic reliability. American Journal of Psychiatry, 136, 815817 .

Spitzer, R. L. \& Williams, J. B. W. (1988). Having a dream: A research strategy for DSM-IV. Archives of General Psychiatry, 45, 871-874. 
Spitzer, R. L., Williams, J. B. W., Gibbon, M., \& First, M. B. (1992). The structured clinical interview for DSMIII-R (SCID): History, rationale, and description. Archives of General Psychiatry, 49, 624-629.

Spitzer, R. L., Williams, J. B. W., Kass, F. , \& Davies, M. (1989). National field trial of the DSM-III-R diagnostic criteria for self-defeating personality disorder. American Journal of Psychiatry, 146, 15611567 .

Sullivan, H. S. (1954). The psychiatric interview. New York: W. W. Norton.

Swain, B., Shisslak, C. M., \& Crago, M. (1991). Clinical features of eating disorders and individual psychological functioning. Journal of Clinical Psychology, 47, 702-708.

Thelen, M. H., Farmer, J., Wonderlich, S., \& Smith, M. (1991). A revision of the bulimia test: The BULIT-R. Journal of Consulting and Clinical Psychology, 3 , 119124 .

Thompson, D. A., Berg, K. M. , \& Shatford, L. A. (1987). The heterogeneity of bulimic symptomatology: Cognitive and behavioral dimensions. International Journal of Eating Disorders, $6,215-234$.

Vanderheyden, D. A., \& Boland, F. J. (1987). A comparison of normals, mild, moderate, and severe binge eaters, and binge vomiters using discriminant function analysis. International Journal of Eating Disorders, 6, 331-337.

Walters, E. E., Neale, M. C., Eaves, L. J., Health, A. C., \& et al. (1993). Bulimia nervosa: A population-based study of purgers versus nonpurgers. International Journal of Eating Disorders, 13, 265-272.

Widiger, T. A., Frances, A. J., Pincus, H. A. \& Davis, W. W. (1991). DSM-IV literature reviews: Rationale, process, and limitations. Journal of Psychopathology and Behavioral Assessment, 12, 189-202.

wiens, A. N. (1990). Structured clinical interviews for adults. In G. Goldstein \& M. Hersen (Eds.), Handbook of psychological assessment (2nd ed.) (pp. 324-341). New York: Pergamon Press. 
Wiens, A. N. \& Matarazzo, J. D. (1983). Diagnostic interviewing. In M. Hersen, A. E. Kazdin, \& A. S. Bellack, A. S. (Eds.), The clinical psychology handbook (pp. 309-328). New York: Pergamon Press.

Williams, J. B. W., \& Spitzer, R. L. (1980). DSM-III field trials: Interrater reliability and list of project staff and participants. In DSM-III Diagnostic and Statistical Manual of Mental Disorder (3rd ed.). Washington, DC: American Psychiatric Press, Inc.

Williamson, D. A. (1990). Assessment of eating disorders: obesity, anorexia, and bulimia nervosa. New York: Pergamon Press, Inc.

Williamson, D. A., Davis, C. J., Norris, L., \& Van Buren, D. J. (1990). Development of reliability and validity for a new structured interview for diagnosis of eating disorders. Paper presented at the annual meeting of the Association for the Advancement of Behavior Therapy, San Francisco.

Williamson, D. A., Kelley, M. L., Davis, C. J., Ruggiero, L., \& Blouin, D. C. (1985). Psychopathology of eating disorders: A controlled comparison of bulimic, obese, and normal subjects. Journal of consulting and Clinical Psychology, 53, 161-166.

Willmuth, M. E., Leitenberg, H., Rosen, J. C., \& Cado, S. (1988). A comparison of purging and nonpurging normal weight bulimics. International Journal of Eating Disorders, $\underline{7}, 825-835$.

Wilson, G. T. (1992). Diagnostic criteria for bulimia nervosa. International Journal of Eating Disorders, $11,315-320$.

Wilson, G. T., Nonas, C. A., \& Rosenblum, G. D. (1993). Assessment of binge eating in obese patients. International Journal of Eating Disorders, 13, 25-33.

Wilson, G. T., \& Smith, D. (1989). Assessment of bulimia nervosa: An evaluation of the eating disorders examination. International Journal of Eating Disorders, $8,173-179$.

Wilson, G. T. \& Walsh, B. T. (1991). Eating disorders. Journal of Abnormal Psychology, 100, 362-365. 
Yager, J., Landsverk, J., \& Edelstein, C. K. (1987). A 20month follow-up study of 628 women with eating

disorders, 1: Course and severity. American Journal of Psychiatry, 144, 1172-1177. 
Appendix A

Interview for Diagnosis of Eating Disorders-IV (IDED-IV) 
Interview for Diagnosis of

Eating Disorders-IV (IDED-IV)

I. Demographic Information

DATE

NAME

AGE

RACE

DATE OF BIRTH

HEIGHT WEIGHT

ADDRESS

HOME PHONE WORK PHONE

(*If adolescent, ask for mother and father's occupation and education)

OCCUPATION EDUCATION

SPOUSE'S OCCUPATION SPOUSE'S EDUCATION

REFERRED BY

GENERAL PHYSICIAN

PSYCHIATRIST

THERAPIST (S)

MEDICATIONS

Prescription

Nonprescription

INSURANCE

PREVIOUS TREATMENT

$\begin{array}{lll}\text { Professionall } & \text { Treatment Period } & \text { Issues Addressed } \\ 1 . & 1 . & 1 . \\ 2 . & 2 \cdot & 2 \cdot \\ 3 . & 3 \cdot & 3 \cdot \\ 4 . & 4 . & 4 .\end{array}$




\section{II.- Detailed Instructions for Adminfstering tho IDED-IV}

The IDED-IV begins with an overview section that follows the general structure of a clinical diagnostic interview. First, basic demographic information is obtained. This section is followed by an open-ended history of eating-disordered symptoms, a description of the chief complaint, and general questions about current functioning. Beginning with nonthreatening demographic questions, allows the interviewer an opportunity to establish rapport. The subject is then encouraged to describe the history of the present illness which lays the groundwork for the more structured diagnostic sections that follow the overview. The overview concludes with general questions about current functioning which refocus the subject on his or her current condition and provide a natural transition to the diagnostic sections. By the time the overview is completed, the interviewer should have obtained enough information to make a tentative differential diagnosis before systematically inquiring about specific symptoms in the diagnostic sections that follow.

The diagnostic sections each begin with a heading intended to cue the interviewer with regard to the DSM-IV diagnostic criterion being rated at the end of each set of questions. Ratings should be made based on subjects' responses to the questions that precede each diagnostic criterion. The majority of items are rated on a 5 point scale, on which either frequency or severity are rated. Ratings should be made as the interview proceeds (although the interviewer has the option to revise ratings if conflicting data are found later in the interview). A rating of 3 or more on each of the symptoms is the operational definition for concluding that the subject has endorsed the presence of that diagnostic symptom. Due to the overlap in symptomatology across eating disorder diagnostic categories, some ratings have been replaced with instructions for transferring information to the diagnostic checklist found at the end of the interview schedule. In order to reduce redundancy in the interview's content and any related inconvenience to the subject, the interviewer is responsible for completing the diagnostic checklist according to the instructions provided throughout the interview and summarized within the diagnostic checklist. To promote the flow of the interview, it is recommended that transfer of information from the interview to the DSM-IV diagnostic checklist (located at the end of the interview protocol) be completed after the interview has been completed. 


\section{Brief Instructions for Administering the IDED-IV}

Interview the subject using each of the questions in the structured interview. Make notes of the subject's answers. As you proceed with the interview, score each of the rating scales by circling the number of the description that best matches the subject's answers to your questions. If there is some doubt as to the best rating, you should ask additional questions to clarify your doubt. After completing the interview, use the eating disorder symptom checklists for DSM-IV diagnostic criteria in order to establish a diagnosis. A rating of 3 or more on each of the symptoms is the operational definition for concluding that the person has endorsed the presence of that diagnostic symptom. 


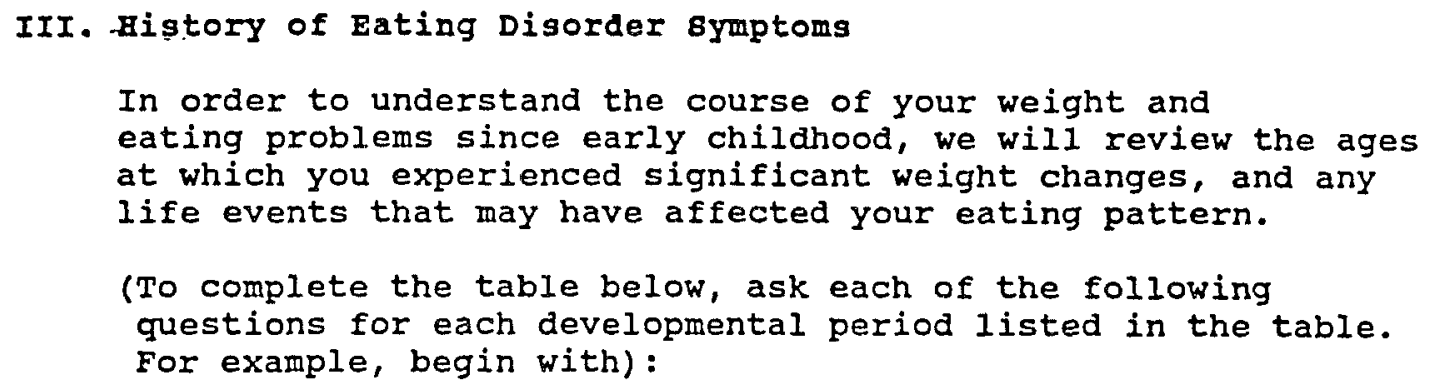

a. "During your childhood years, what weight changes did you experience?" (note significant ages, and overall highest and lowest adult weight)

b. "What life events may have affected these weight changes?"

c. "How did these life events affect your eating pattern?"

Period

Age/Weight Life Events

Effects on Eating

Childhood

(Birth-12 yrs) 


Period Age/Weight Life Events Effects on Eating
$\frac{\text { Young Adulthood }}{(20-34 \text { yrs })}$

Middle Adul thood

(35-49 Yrs)

Late Adul thood

(50 yrs \& older) 


\section{IV. current status of Eating Disorder Bymptoms}

\section{A. Eating pattern}

1. What are your current concerns regarding your eating and your weight?

2. Would you describe an example of the types and amounts of foods you might eat in a typical day? while dieting?

Breakfast:

Snack:

Lunch:

Snack:

Dinner:

Snack: 
B. Medical Problems

1. Have you had any medical problems? $Y N$ (Check for dizziness, LBP, HBP, thyroid problems, diabetes.)

2. Have you had any dental problems? $Y$ N (e.g., tooth erosion.) (If yes, describe.)

\section{c. Family Information}

1. How many members are there in your household?

2. Do they know about your eating problems? $\mathbf{Y} N$ (If yes, how do they react/feel about your eating disorder?)

3. Who would be available to participate in your treatment?

4. Has anyone in your family had an eating disorder? weight problems? obesity? (If yes, describe.)

5. Has anyone in your family experienced psychiatric problems? (If yes, describe.) 
VI. Questions for the Diagnosis of Anorexia Nervosa

A. Refusal to Maintain Normal Body weight

1. Do you currently go periods of time without eating (starvation) to control your weight? $\mathrm{Y}$ N (If yes, describe.)

2. When did you first begin to lose weight by restricting your eating?

3. Are there any factors/situations which seem to increase your periods of restrictive eating?

4. Are there any factors/situations which seem to decrease your periods of restrictive eating?

5. What is your goal weight?

(Rating of 3 or higher meets criterion.)

A. Refusal to maintain appropriate welght for height (you may use height/weight charts to assist in maxing this rating)

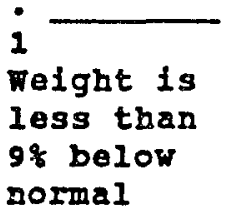

normal

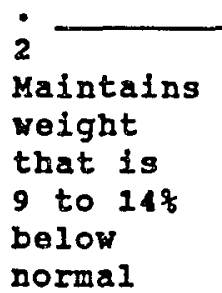

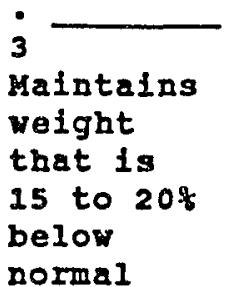

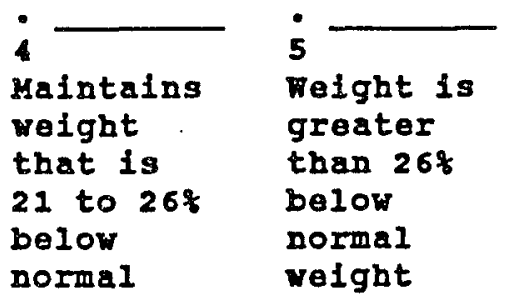




\section{B. Fear of weight Gain \\ 1. Do you feel that your weight is normal? $\mathrm{Y}$ N (Describe.)}

2. How often do you weigh yourself?

3. What emotional reaction would you have if you gained

2 Ibs.?

5 Ibs.?

10 Ibs.?

(Rating of 3 or nigher meets criterion.)

B. Intense fear of weight gain

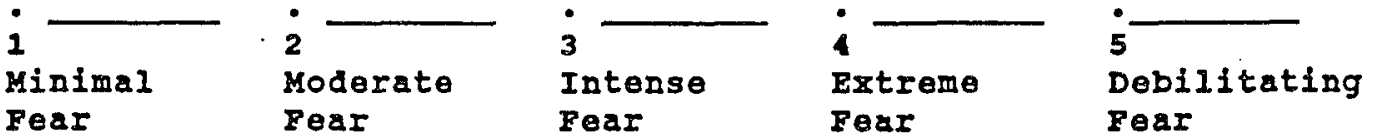

c. Disturbance of Body Image

1. Do you wish to be thinner than you are now? $Y \quad N$ (If yes, ask what body areas should be thinner.)

2. Do you think or worry a lot about your weight and body size? $Y$ N (Describe). 
- 3. Do you ever feel fat? $Y$ N
(If yes, ask when do you feel fat?)

(Rating of 3 or higher meets criterion.)

c. Disturbance of body Image

(1) body lmage disturbance: Feels "fat" oven if not significantly overweight

:

1

Never

or not

applicable

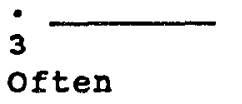

Often

sometimes

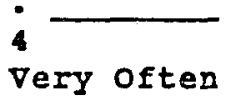

very often $\mathbf{5}$

All of the time

4. How often does your body size affect the way you feel about yourself?

(Rating of 3 or higher meets criterion.)

c. Disturbance of body image

(2) undue influence of body veight/ghape on self-evaluation
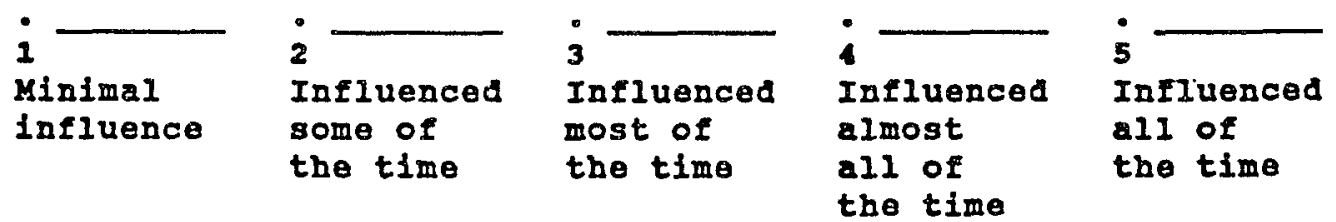
5. Do you feel that your current weight is creating any problems for you? (e.g., medical, emotional, family)

(Rating of 3 or higher meets criterion.)

c. Disturbance of body image

(3) denial of seriougness of current low body weight

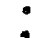

No

denial

or not

applicable$$
2
$$

some

denial
3

Moderate

denial
:

Btrong

denial
5

Extreme

denial

\section{Menstrual Irregularities}

1. When was your last menstrual period?

2. Have you experienced any menstrual irregularities (skipped period, lighter flow, shorter number of days)? $Y$ N (If yes, describe type of irregularity and for how long).

3. If yes, are there any medical reasons for these menstrual irregularities? (Describe.) 
- 4. Are you taking any hormone medication (e.g., birth control pills, estrogen)? $Y N$ (If yes, ask how long have you been taking this medication?)

5. Have you found that your menstrual periods cease when you stop taking birth control pills or hormone replacement medication? $Y$ N (Describe.)

6. For how many consecutive months have you not had your menstrual period?

(Rating of 3 or higher meets criterion.)

D. Amenorrhea

i

Very

Regular

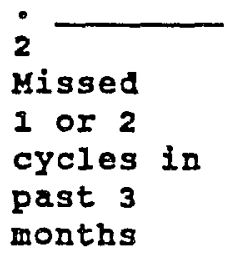

; Missed 1 or 2 past 3 months

3
Missed 3
consecutive
cycles in
past 3
months

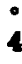
Missed 4 or 5 consecutive cycles in 5 months

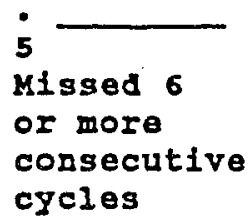

5 cycles

v. Questions for Binge Eating Disorder

A. Binge Eating (Recurrent Episodes)

1. Do you ever binge (rapidly consume a large amount of food in a discrete period of time)? $Y$ N 
- 2. What kind of foods do you eat during a binge and how much?

3. Do you ever feel as though you have overeaten when you eat small portions of certain fattening foods? $Y \mathrm{~N}$ (Describe.)

4. When do your binge eating episodes occur?

(during meals, after meals, throughout day, etc.)

5. How long does each binge usually last?

(Rating of 3 or higher meets criterion.)

A. Recurrent episodes of binge eating

(1) eating a large amount of food in a discrete period of time

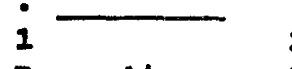

Doesn't

binge

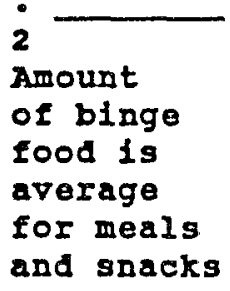

-

of bingo

rood is

typlcally

larger

than normal

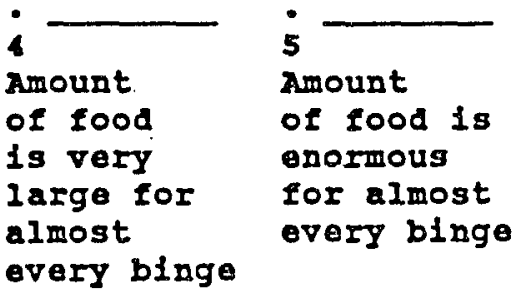


A. Binge eating (Loss of control)

1. Do you feel you can stop eating once a binge has begun? $Y \quad N$ (Describe.)

2. How often do you feel out of control of your eating during a binge?

(Rating of 3 or more meets criterion.)

A. Recurrent binge-eating episodes

(2) Feeling of loss of control of eating during a bingo

$\begin{array}{lllll}\text { i } & \text { i } & \text { is } & & \\ \text { Alvays } & \text { occa- } & \text { Prequent } & \text { Almost } & \text { Never in } \\ \text { In } & \text { sional } & \text { loss of } & \text { always } & \text { control } \\ \text { control } & \text { loss of } & \text { control } & \text { out of } & \\ & \text { control } & & & \text { control }\end{array}$

B. Behavioral Indicators

1. When binge eating, do you feel your eating is more rapid than normal? $Y$ N (Describe.) 
(Rating of 3 or higher meets criterion.)

B. Behavioral indicator

(1) rapid eating during binges

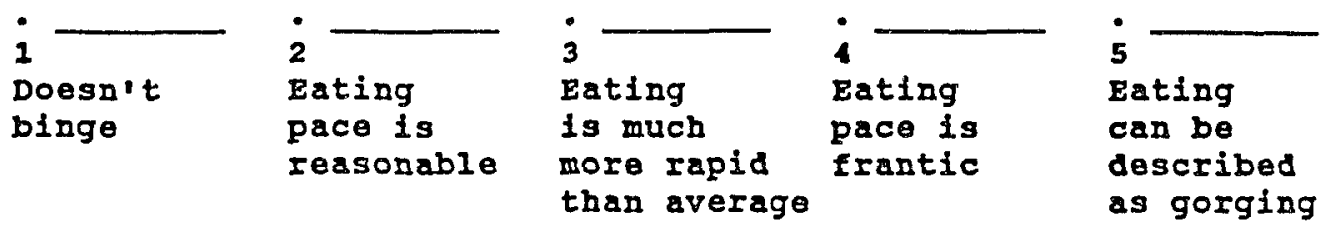

2. When binge eating, how often do you eat enough to feel uncomfortably full? (Describe.)

(Rating of 3 or higher meets criterion.)

B. Behavioral indicator

(2) eating until feeling uncomfortably full whon binging

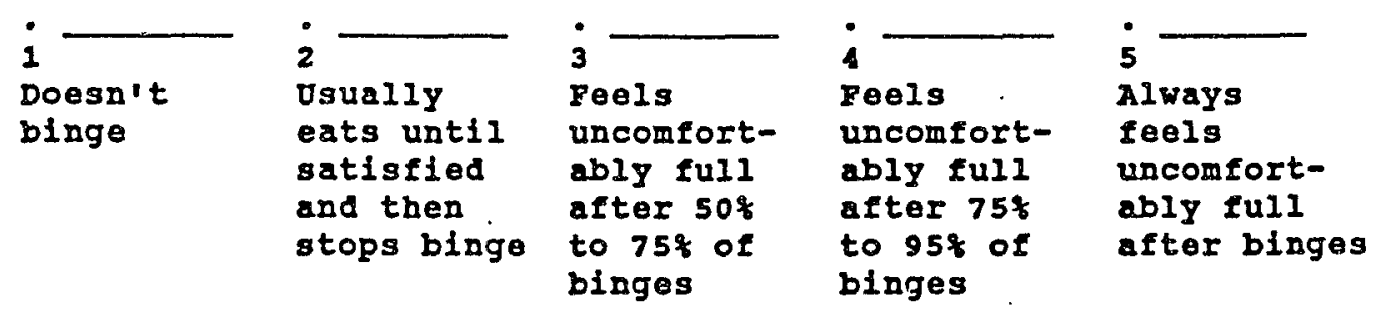


3. How often do you eat large amounts of food when you don't -. really feel hungry? (Describe.)

(Rating of 3 or higher meets criterion.)

B. Behavioral indicator

(3) oating largo amounts of food when not hungry

$\begin{array}{lllll}\dot{1} & \dot{2} & \dot{3} & \dot{4} & \text { - } \\ \text { Seldom } & 1-7 & 2 \text { days/ } & 2-6 & \text { At least } \\ \text { eats when } & \text { days/ } & \text { week } & \text { days/ } & \text { once/day } \\ \text { not hungry month } & & \text { week } & \end{array}$

4. How often do you binge alone, or in secret? Why? (check for embarrassment).

(Rating of 3 or higher meets criterion.)

B. Behavioral indicator

(4) eating alone while binging due to embarrassment

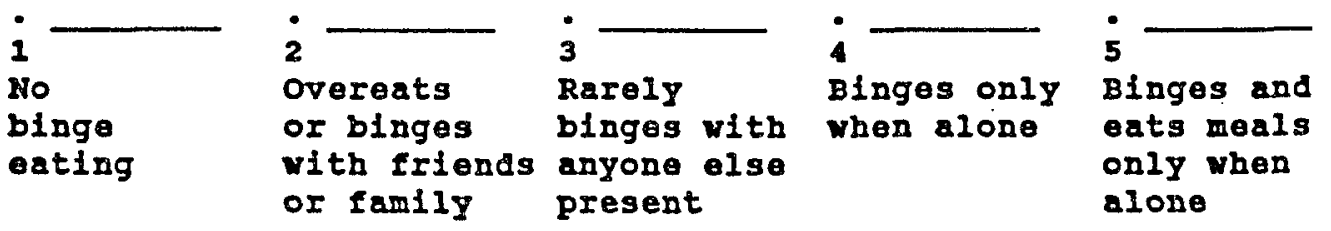


5. Are there any factors which appear to increase

the frequency of your binge eating?

6. Are there any factors which appear to decrease the frequency of your binge eating?

7. What emotions do you typically experience before, during, and after a binge? (check for disgust, depression, guilt)

a. before:

b. during:

c. after:

(Rating of 3 or higher meets criterion.)

B. Behavioral indicator

(5) negative affect (disgusted, depressed, very guilty) after binge eating

\begin{tabular}{|c|c|c|c|c|}
\hline $\begin{array}{l}\text { Doesn't } \\
\text { binge }\end{array}$ & $\begin{array}{l}\text { Minimal } \\
\text { negative } \\
\text { affect } \\
\text { post-binge }\end{array}$ & $\begin{array}{l}\text { Moderate } \\
\text { negative } \\
\text { affect } \\
\text { post-binge }\end{array}$ & $\begin{array}{l}\text { severe } \\
\text { negative } \\
\text { affect } \\
\text { post-binge }\end{array}$ & $\begin{array}{l}\text { Debilitating } \\
\text { negative } \\
\text { affect } \\
\text { post-binge }\end{array}$ \\
\hline
\end{tabular}




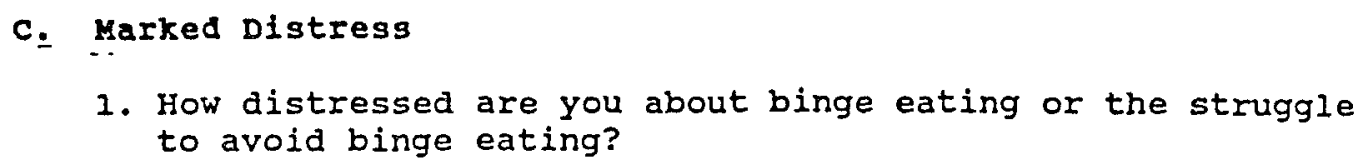

(Rating of 3 or higher meets criterion)

c. Distress regarding binge eating

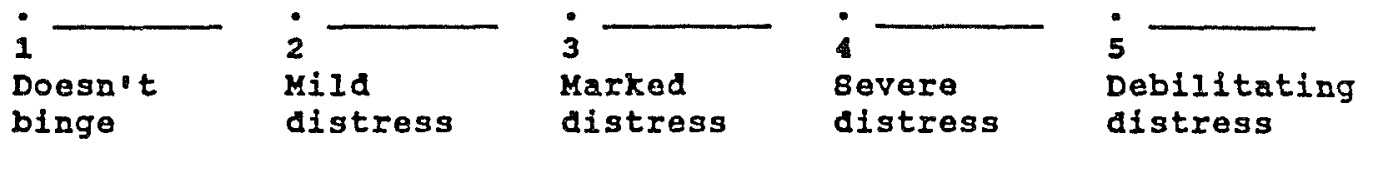

D. Frequency of Binge Eating

1. On average, how frequently do you binge, and how long have you been binging at that rate?

\section{Frequency Time Frame}
a. per day?
b. per week?
c. per month?

2. How long have you been binging at least two days per week?

D. Frequency of binge eating

1. If individual binging two days per veex or more, for 6 or more months, endorse criterion D for Blnge Eating Disorder on the attached diagnostic checklist (p.27).

2. If individual binging two days per week or more, for 3 or more months, endorse criterion C for Bullmis Nervosa on the attached diagnostic chocklist (p.28). 
E. To meet the criteria for Binge Eating Disorder, patient must have absence of:

1. Inappropriate compensatory Behavior

(See pp. 21-23, rating of 1 for Rating $B(1,2,3, \& 4)$ meets criterion. Transfer value to diagnostic checklist, $p$. 28, Criterion E for Binge Eating Disorder.)

2. Bulimia Nervosa (derive from results of diagnostic checklist, p. 28-29)

3. Anorexia Nervosa (derive from results of diagnostic checklist, p. 26-27)

VII. Bulimia Nervosa

(To make rating see p. 13, rating $A$ (1))

A. Recurrent binge-eating episodes

(1) eating large amount of food in a discrete period of time

$\dot{1}$

Doesn't binge

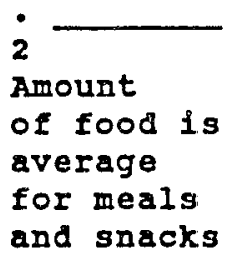

3

Anount

of food is

typically

larger

than

normal

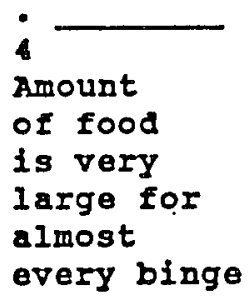

5

Amount

of food is

enormous

for almost

every binge 
(To make rating see p. 14 , rating $A(2)$ )

- .

A. Recurrent binge-eating episodes

(2) Teeling of loss of control of eating during a binge

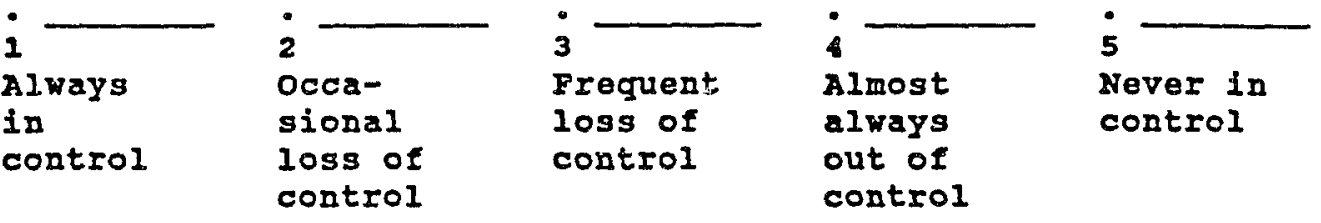

B. Compensatory Behavior

1. Do you purge after meals or after a binge (vomit, abuse laxatives or diuretics)? $\mathrm{Y} \quad \mathrm{N}$

2. When did you first begin to purge?

3. Are there any factors which appear to increase the frequency of purging?

4. Are there any factors which appear to decrease the frequency of purging?

5. Do you purge by vomiting? $\mathrm{Y} \quad \mathrm{N}$ (If yes, ask how often do you purge by vomiting?) 
(Rating of 3 or higher meets criterion)

B. Compensatory Behavior

(1) self-induced vomiting

1

None
2

vomits

a Iew

times/

year
3

Vomits

several

times/

month i

Vomits

several

times/

week
5

Vomits 1

or more

times/

day

6. Do you purge by using laxatives? $\mathrm{Y} N \mathrm{~N}$

(If yes, ask what type and how often)

7. Do you purge by using diuretics (i.e., water pills)? $\mathrm{Y} N$ (If yes, ask what type and how often)

(Rating of 3 or higher meets criterion.)

B. Compensatory Behavior

(2) laxative/diuretic abuse (an episode is defined as taking 1 or more pilis during a short time interval to rid body of food)

1

None

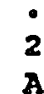

A few times/ year
3

several

times/

month

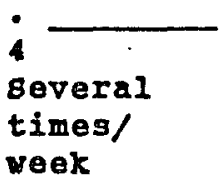

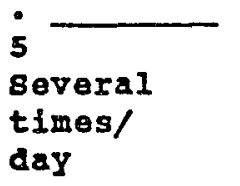

8. Do you often go on strict diets (rigid eating, skipping meals, eating virtually nothing on a given day)? (Describe.) 
9. Do you use diet pills to lose weight? $\mathrm{Y}$ N

-. (If yes, ask what type and how often?)

10. When was the last time you took a diet pill?

11. How often do you engage in strict dieting?

(Rating of 3 or higher meets criterion.)

B. Compensatory Behavior

(3) dieting/fasting

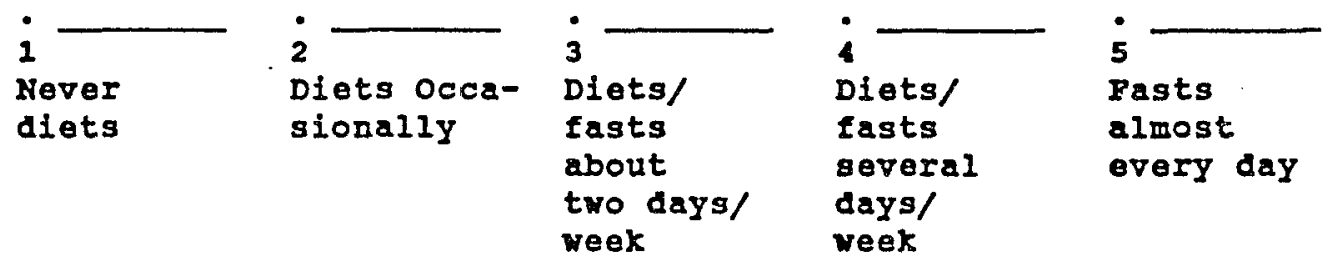

12. Do you engage in excessive exercise to control your weight? (intense exercise which is compulsive or obligatory from person's description aimed at ridding body of food/calories.) $Y$ N

(If yes, ask what type and how often.) 
(Rating of 3 or higher meets criterion.)

B. Compensatory Behavior

(1) excessive exercise (exercise which is compulsive or obligatory from person's description aimed at ridding body of food/calories)

9

Exercises

to promote health
:

Exercises

to $105 \theta$

weight
3

Exercises

to rid body

of EOOd/

calories

2-4 days

per week i

Exercises Exercises

to rid body more than

of food/

calories

5-7 days

per week

c. Frequency of binge eating over past 3 months

Bee individual's response to p. 18 , criterion D, question 2 . If individual meets criterion indicate on diagnostic checklist (p. 28, Criterion ()).

(To make rating see p. 10, Rating $c(2)$ )

D. Undue influence of body weight/shape on self-evaluation

1

Minimal

influence

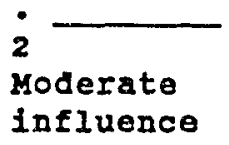

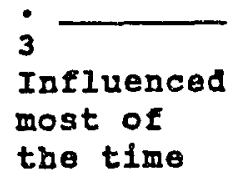

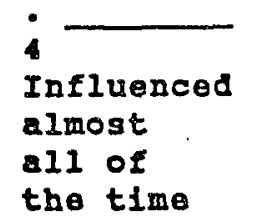

5

Influenced

all of

the time 
VIII. Eating Disorder Not otherwise speciried

- .

A-C. For Eating Disorder Not Otherwise Bpecified categories 1, 2

and 3 , see instructions for each category on pp. 29-30.

D. Purging Small Amounts of Food

1. Do you ever purge (i.e., vomit, use laxatives/diuretics, or excessively exercise) after eating small amounts of food (e.g., two cookies)? $Y$ N (Describe.)

2. If yes, how often do you purge small amounts of food, and for how long have you engaged in this behavior?

(If patient is normal weight, response to question AI is "YES", and response to question $A 2$ is "at least two days per week, for at least 3 months", endorse ED Nos eating pattern number 4, p.30).

\section{E. Tasting}

1. Have you ever attempted to control your weight by chewing, spitting out, and not swallowing large amounts of food? $\mathrm{Y} N$ (Describe).

2. If yes, how often do you purge small amounts of food, and for how long have you engaged in this behavior?

(If response to question B1 is "YES", and response to question B2 is "at least two days per week, for at least 3 months", endorse ED NOS eating pattern number 5, p.30). 


\section{Other Eating Problems}

- -

A. Night Binging

1. Do you have a problem with binge eating primarily late in the evening? $Y$ N (Describe.)

2. Do you awaken and binge during the night? $Y N$ (Describe.)

3. When binging during the evening, do you find yourself in a conscious or semi-conscious state? (Describe.)

4. How often do you binge at night, and for how long has this been a problem for you?

(If response to question $A 1$ OR $A 2$ is "YES", and response to question $A 4$ is "at least two days per week, for at least 3 months", endorse Night Binging, p.30). 


\section{x. Diagnostic Checklists for the Eating Disorders}

To derive the relevant eating disorder diagnoses, detach the following checklists, and review the ratings made within the IDED-IV interview. Check each criterion rated 3 or more on the rating scale pertaining to each symptom, and/or see special instructions where noted.

\subsection{Anorexia Nervosa}

A. Refusal to maintain body weight at or above a minimally normal weight for age and height $(e . g .$, weight loss leading to maintenance of body weight less than $85 \%$ of that expected; or failure to make expected weight gain during period of growth, leading to body weight less than 858 of that expected).

B. Intense fear of gaining weight or becoming fat, even though underweight.

c. Disturbance in the way in which one's body weight or shape is experienced; undue influence of body weight or shape on self-evaluation, or denial of the seriousness of the current low body weight.

(**Meets this criterion if Ratings $c(1,2$, or 3 ) on $\mathrm{pp}$. 10-11, rated 3 or higher.)

D. In post-menarcheal females, amenoxrhea, i.e., the absence of at least three consecutive menstrual cycles. (A woman is considered to have amenorrhea if her periods occur only following hormone, e.g., estrogen, administration.)

(**Meets this criterion if rating 3 or higher for Rating $D$, p. 12 OR if "YES" response to question 5, p. 12.)

Specify type:

(If person meets criteria for anorexia nervosa, check the type which is most descriptive.)

Restricting type: During the episode of Anorexia Nervosa, the person does not regularly engage in binge eating or purging behavior (i.e., self-induced vomiting or the misuse of laxatives or diuretics.)

(**Look for rating of 2 or lower on Pp. 13-14, Ratings $A 1$ and A2 AND on p.21, Ratings B1 and B2.) 
Binge Eating/Purging type: During the episode of Anorexia Nervosa, the person regularly engages in binge eating or purging behavior ( $i . e .$, self-induced vomiting or the misuse - of laxatives or diuretics.)

(**Look for rating of 3 or higher on pp. 13-14, Ratings $A I$ and $A 2 O R$ on $\mathrm{O} .21$, Ratings $\mathrm{BI}$ and $\mathrm{B2}$. )

\section{Binge Eating Disorder}

(Check symptoms that were rated 3 or more on the rating scales pertaining to each symptom. **See special instructions where noted.)

A. Recurrent episodes of binge eating. An episode of binge eating is characterized by both of the following:

(1) eating, in a discrete period of time (e.g., within any 2 hour period), an amount of food that is definitely larger than most people would eat during a similar period of time under similar circumstances, and,

(2) a sense of lack of control over eating during the episode (e.g., a feeling that one cannot stop eating or control what or how much one is eating).

B. The binge eating episodes are associated with at least three of the following:

(1) eating much more rapidly than normal;

(2) eating until feeling uncomfortably full;

(3) eating large amounts of food when not feeling physically hungry;

(4) eating alone because of being embarrassed by how much one is eating;

(5) feeling disgusted with oneself, depressed, or very guilty after overeating.

c. Marked distress regarding binge eating.

D. The binge eating occurs, on average, at least two days a week for 6 months.

(**To endorse criterion, see response to question D1, p. 18.) 
E. The binge eating is not associated with the use of inappropriate compensatory behaviors (e.g., purging, fasting, excessive exercise), and does not occur exclusively during

- the course of Anorexia Nervosa or Bulimia Nervosa.

( $* *$ To meet criterion, $100 k$ for rating of 1 for Ratings $B$ $(1,2,3$, AND 4), on pp. 21-23. In addition, see checklist to determine whether criteria for Arorexia Nervosa (p. 26-27) and Bulimia Nervosa (p. 28-29) have been met.)

\subsection{Bulimia Nervosa}

(Check symptoms that were rated 3 or more on the rating scales pertaining to each symptom. *tSee special instructions where noted.)

A. Recurrent episodes of binge eating. An episode of binge eating is characterized by both of the following:

(Meets this criteria, if (1) \& (2) below were rated 3 or more.)

(1) eating, in a discrete period of time (e.g., within any two hour period), an amount of food that is definitely larger than most people would eat during a similar period of time and under similar circumstances, and,

( $* *$ Rating of 3 or higher on $p .13$, Rating $A$ (1) meets criterion.)

(2) a sense of lack of control over eating during the episode (e.g., a feeling that one cannot stop eating or control what or how much one is eating)

(**Rating of 3 or higher on p. 14, Rating A (2) meets criterion.)

B. Recurrent inappropriate compensatory behavior in order to prevent weight gain, such as: self-induced vomiting; misuse of laxatives, diuretics or other medications; fasting; or excessive exercise.

( $*$ Rating of 3 or higher on pp. 21-23, Ratings $B(1,2,3$, OR 4) meets criterion.)

c. The binge eating and inappropriate compensatory behaviors both occur, on average, at least two days per week for three months.

( $\star \star$ See response to p. 18 , Criterion D2). 
D. Self-evaluation is unduly influenced by body shape and weight.

- -. (Rating of 3 or higher on p. 10, Rating c (2), meets criterion.)

E. The disturbance does not occur exclusively during episodes of Anorexia Nervosa.

(**see results of diagnostic checklist, p. 26-27.)

specify type:

(If the person meets criteria for Bulimia Nervosa, check the type which is most descriptive)

Purging type: the person regularly engages in self-induced vomiting or the misuse of laxatives or diuretics.

(** Look for rating of 3 or higher on Rating $B(1)$, p. 21 OR Rating B (2), p. 21.)

Nonpurging type: the person uses other inappropriate compensatory behaviors such as fasting or excessive exercise, but does not regularly engage in self-induced vomiting or the misuse of laxatives or diuretics.

(**Look for rating of 3 or higher on p. 22 , Rating $B$ (3) and p. 23, Rating $B$ (4) AND rating of 2 or less on $p .21$ on Rating $B$ (1) and p. 21, Rating B (2).)

307.50 Eating Disorder Not Otherwise specified (ED NOS)

If the subject does not meet the criteria for a diagnosis of anorexia nervosa, bulimia nervosa, or binge eating disorder, determine whether any of the following descriptions of Eating Disorder Nos apply.

This category is for disorders of eating that do not meet the criteria for any specific Eating Disorder. Examples include:

(1) all of the criteria for Anorexia Nervosa are met except the individual has regular menses (see p. 26)

(2) all of the criteria for Anorexia Nervosa are met except that, despite significant weight loss, the individual's current weight is in the normal range (see p. 26) 
(3) all of the criteria for Bulimia Nervosa are met except binges occur at a frequency of less than twice a week or for a duration of less than three months (see p. 28)

(4) an individual of normal body weight who regularly engages in inappropriate compensatory behavior after eating small amounts of food (e.g., self-induced vomiting after the consumption of two cookies)

(**Meets criterion if on page 24 , response to question DI is "YES" and response to D2 is "for at least two days per week, for at least 3 months", and individual is of normal weight)

(5) an individual who repeatedly chews and spits out, but does not swallow, large amounts of food (i.e., rasting)

(**Meets criterion if on page 24 , response to question $\mathrm{El}$ is "YES" and response to E2 is "for at least two days per week, for at least 3 months".)

Other Eating Problems:

Night Binging: an individual who binges late in the evening, or awakens repeatedly to binge, while in either a conscious or semi-conscious state.

(** Meets criterion if on page 25, response to question Al or $A 2$ is "YES" and response to A4 is "for at least two days per week, for at least 3 months".) 
Appendix B

Consent Form 


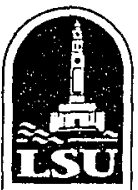

LOU I S I A N A STATE UN I VER S IT Y

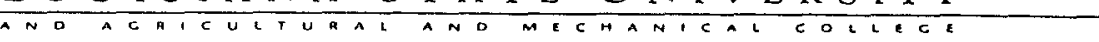
Psychological Services Center - Department of Psychology

\section{Consent Form}

This study is being conducted by Donald Williamson, Ph.D., and Vesna Kutlesic, M. A., of the Psychology Department at Louisiana State University. The purpose of this study is to examine eating attitudes and behaviors among adolescent and adult females. All responses to the questionnaires will remain strictly confidential. Only the researchers will have access to gathered information, and the questionnaires will be coded by number not by name. All results are based on group responses, not the responses of single individuals. If you are interested, we would be glad to provide you with the results of the study. This research may be used for published work.

You will be asked to participate in a 45 minute interview, and to complete several questionnaires which will require an additional 15 minutes. If you have any questions, feel free to ask the researcher. Your participation is completely voluntary. If you decide to participate, please sign this form and fill out the attached questionnaires. You may withdraw from the study at any time.

Thank you for your participation.

Participant's Signature

Date

Witness

Date

Yes, I am interested in the results of your study, my address is: 


\section{Appendix C \\ Eating Attitudes Test (EAT)*}

* Permission to reproduce measure granted by author, David M. Garner, Ph.D., Neurobehavioral Associates, 4632 Okemos Road, Okemos, MI 48864. See Appendix F for copy of letter. 
D.M. Garner and P.E. Garfinkel

"EAT"

Please clrcle the response which best applies to each of the numbered statements. Please answer each question carefully. Thank you,

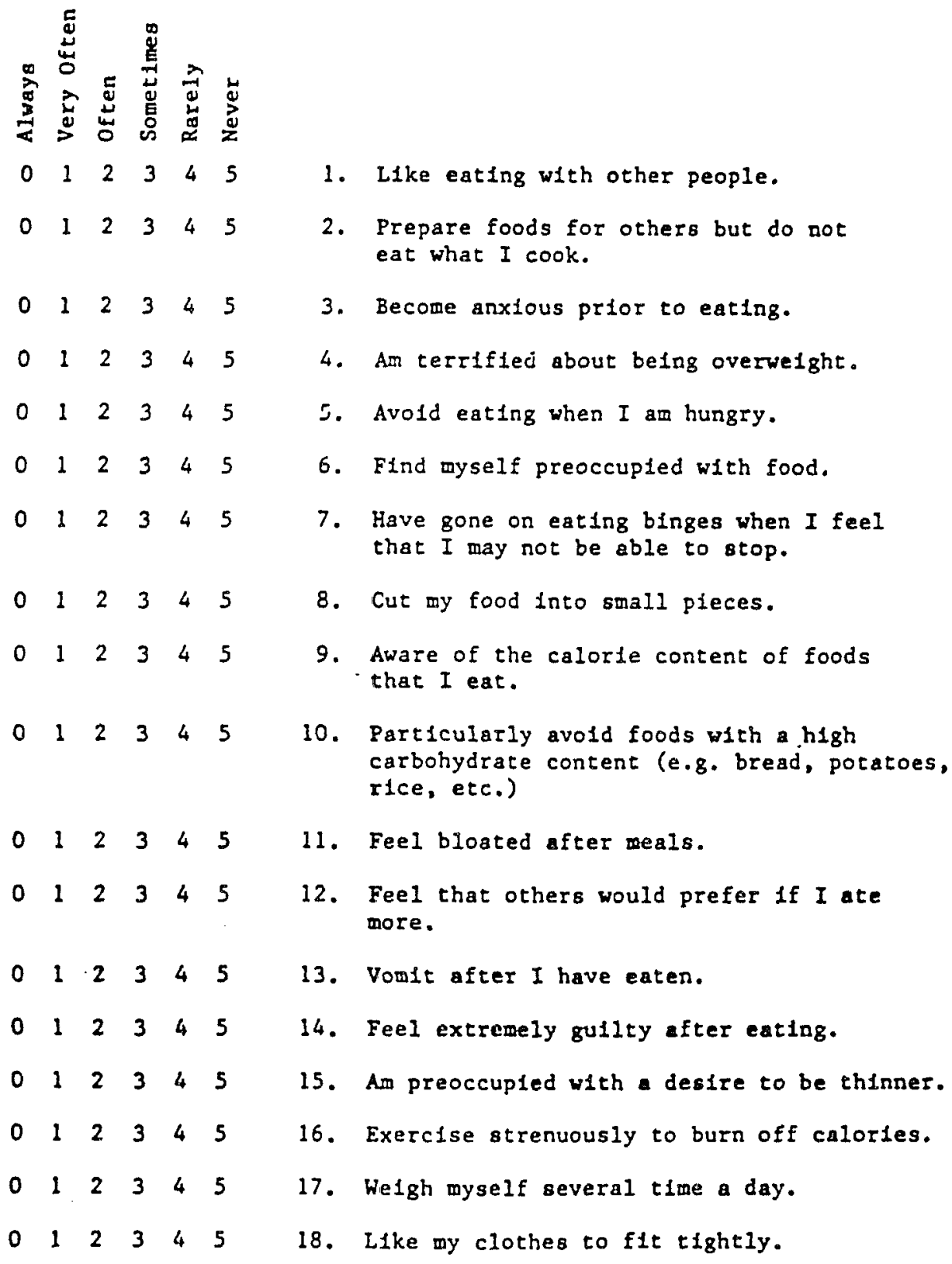




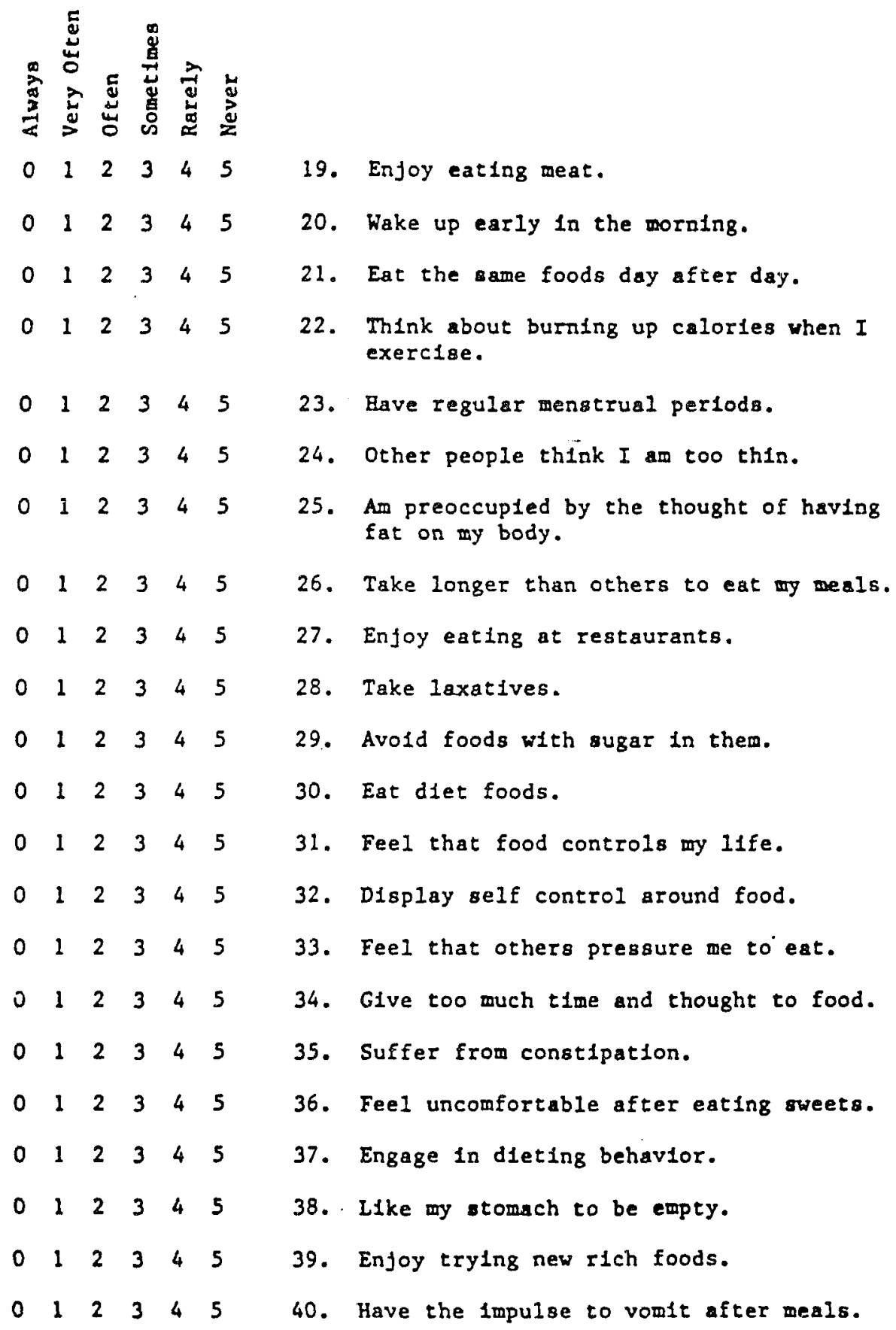


Appendix D

Bulimia Test-Revised (BULIT-R)*

* Permission to reproduce measure granted by author, Mark $\mathrm{H}$. Thelen, Ph.D., Department of Psychology, University of Missouri-Columbia, Columbia, Missouri 65211. See Appendix $F$ for copy of letter. 
The BULIT-R

Answer each question by circling the appropriate answer below. Please respond to each item as honestly as possible; remember all of the information you provide will be kept strictly confidential.

1. I am satisfied with my.eating patterns.
1. agree
2. neutral
3. disagree a little
4. disagree
5. disagree strongly

2. Would you presently call yourself a "binge eater"?
1. yes, absolutely
2. yes
3. yes, probably
4. yes, possibly
5. no, probably not

3. Do you feel you have control over the amount of food you consume?

1. most or all of the time

2. a lot of the time

3. occasionally.

4. rarely

5. never

4. I am satisfied with the shape and size of my body.
1. frequently or always
2. sometimes
3. occasionally
4. rarely
5. seldom or never

5. When I feel that my eating behavior is out of control, I try to take rather extreme measures to get back on course (strict dieting, fasting, laxatives, diuretics, self-induced vomiting, or vigorous exercise).

1. always

2. almost always

3. frequently

4. sometimes

5. never or my eating behavior is never out of control 
6. I use laxatives or suppositories to help control my weight.

1. once a day or more

2. 3-6 times a week

3 . once or twice a week

4. 2-3 times a month

5. once a month or less (or never)

7. I am obsessed about the size and shape of my body.
1. always
2. almost always
3. frequently
4. sometimes
5. seldom or never

8. There are times when I rapidly eat a very large amount of food.

1. more than twice a week

2. twice a week

3. once a week

4. 2-3 times a month

5. once a month or less (or never)

9. How long have you been binge eating (eating uncontrollably to the point of stuffing yourself)?

1. not applicable; I don't binge eat

2. less than 3 months

3. 3 months -1 year

4. 1 - 3 years

5. 3 or more years

10. Most people I know would be amazed if they knew how much food I can consume at one sitting.
1. without a doubt
2. very probably
3. probably
4. possibly
5. no

11. I exercise in order to burn calories.

1. more than 2 hours per day

2. about 2 hours per day

3. more than 1 but less than 2 hours per day

4. One hour or less per day

5. I exercise but not to burn calories or I don't exercise 
12. Gompared with women your age, how preoccupied are you about your weight and body shape?

1. a great deal more than average

2. much more than average

3. more than average

4. a little more than average

5. average or less than average

13. I am afraid to eat anything for fear that I won't be able to stop.
1. always
2. aimost always
3. frequently
4. sometimes
5. seldom or never

14. I feel tormented by the idea that I am fat or might gain weight.
1. always
2. almost always
3. frequently
4. sometimes
5. seldom or never

15. How often do you intentionally vomit after eating?
1. 2 or more times a week
2. once a week
3. 2-3 times a month
4. once a month
5. less than once a month or never

16. I eat a lot of food when I'm not even hungry.
1. very frequently
2. frequently
3. occasionally
4. sometimes
5. seldom or never

17. My eating patterns are different from the eating patterns of most people.

1. always

2. almost always

3. frequently

4. sometimes

5. seldom or never 
18. After I binge eat I turn to one of several strict methods to try to keep from gaining weight (vigorous exercise, strict dieting, fasting, self-induced vomiting, laxatives, or diuretics).

1. never or I don't binge eat

2. rarely

3. occasionally

4. a lot of the time

5. most or all of the time

19. I have tried to lose weight by fasting or going on strict diets.

1. not in the past year

2. once in the past year

3. 2-3 times in the past year

4. 4-5 times in the past year

5. more than 5 times in the past year

20. I exercise vigorously and for long periods of time in order to burn calories.

1. average or less than average

2. a little more than average

3. more than average

4. much more than average

.5. a great deal more than average

21. When engaged in an eating binge, 1 tend to eat foods that are high in carbohydrates (sweets and starches).

1. always

2. almost always

3. frequently

4. sometimes

5. seldom, or I don't binge

22. Compared to most people, my ability to control my eating behavior seems to be:

1. greater than others' ability

2. about the same

3. less

4. much less

5. I have absolutely no control 
23. Lwould presently label myself a 'compulsive eater', (one who engages in episodes of uncontrolled eating).
1. absolutely
2. yes
3. yes, probably
4. yes, possibly
5. no, probably not

24. I hate the way my body looks after I eat too much.
1. seldom or never
2. sometimes
3. frequently
4. almost always
5. always

25. When I am trying to keep from gaining weight, I feel that I have to resort to vigorous exercise, strict dieting, fasting, self-induced vomiting, laxatives, or diuretics.
1. never
2. rarely
3. occasionally
4. a lot of the time
5. most or all of the time

26. Do you believe that it is easier for you to vomit than it is for most people?
1. yes, it's no problem at all for me
2. yes, it's easier
3. yes, it's a little easier
4. about the same
5. no, it's less easy

27. I use diuretics (water pills) to help control my weight.
1. never
2. seldom
3. sometimes
4. frequently
5. very frequently

28. I feel that food controls my life.
1. always
2. almost always
3. frequently
4. sometimes
5. seldom or never 
29. I try to control my weight by eating little or no food for a day or longer.
1. never
2. seldom
3. sometimes
4. frequently
5. very frequently

30. When consuming a large quantity of food, at what rate of speed do you usually eat?

1. more rapidly than most people have ever eaten in their lives

2. a lot more rapidly than most people

3. a little more rapidly than most people

4. about the same rate as most people

5. more slowly than most people (or not applicable)

31. I use laxatives or suppositories to help control my weight.
1. never
2. seldom
3. sometimes
4. frequently
5. very frequently

32. Right after I binge eat I feel:
1. So fat and bloated I can't stand it
2. extremely fat .
3. fat
4. a little fat
5. OK about how my body looks or I never binge eat

33. Compared to other people of my sex, my ability to always feel in control of how much I eat is:
1. about the same or greater
2. a little less
3. less
4. much less
5. a great deal less

34. In the last 3 months, on the average how often did you binge eat (eat uncontrollably to the point of stuffing yourself)?

1. once a month or less (or never)

2. 2-3 times a month

3. once a week

4. twice a week

5. more than twice a week 
35. Most people I know would be surprised at how fat I look after I eat a lot of food.

1. yes, definitely

2. yes

3. yes, probably

4. yes, possibly

5. no, probably not or I never eat a lot of food

36. I use diuretics (water pills) to help control my weight.

1. 3 times a week or more

2. once or twice a week

3. 2-3 times a month

4. once a month

5. never 
Appendix E
Body Image Assessment (BIA) 
194
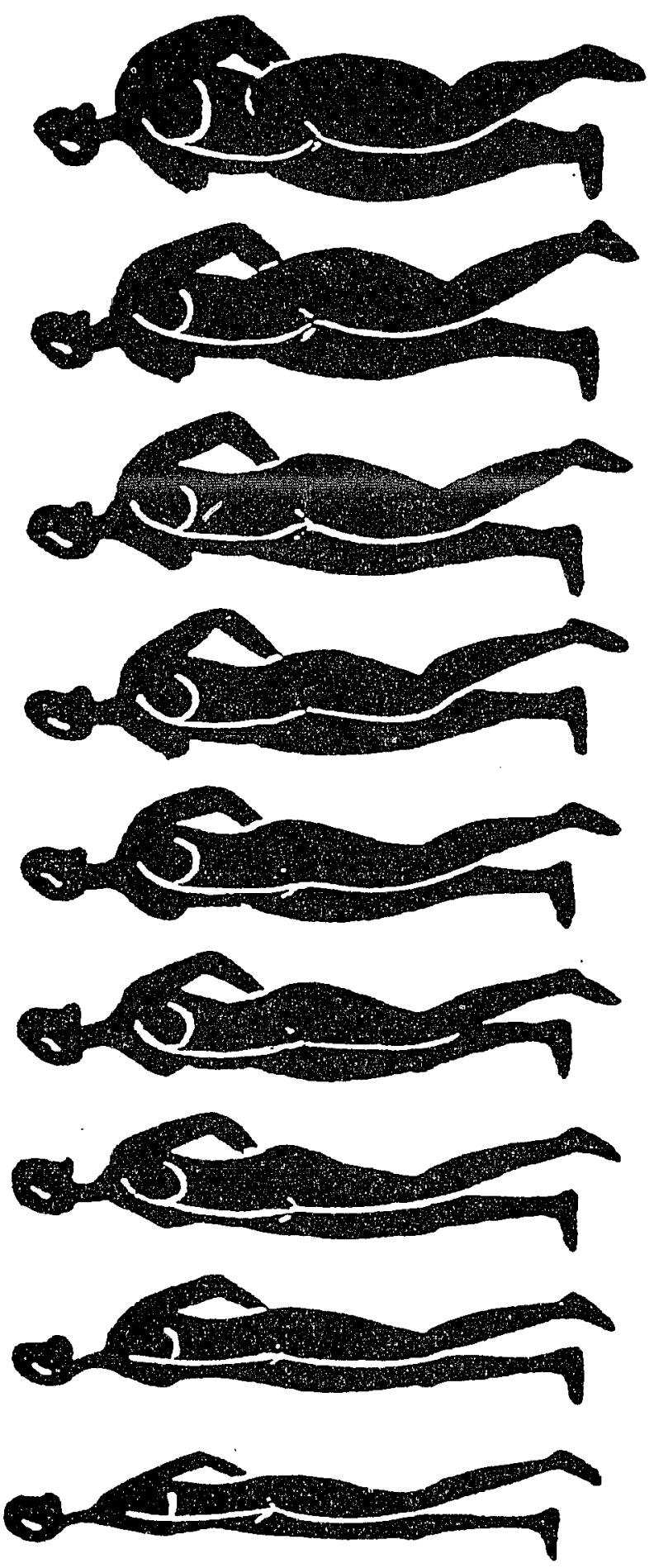
Appendix $F$

Letters of Permission to Reproduce Measures 
NEUROBEHAVIORAL ASSOCIATES

4632 OKEMOS ROAD - OKEMOS, MI 48864 - (5T7) 347-3300) - YAX (5T7) $367-3303$

DAVID M. GARNRR, PH.D.

EXRCUTMS DRECTOR

June 13,1995

Vesna Kutlesic, M.A.

658 N. 8th Street, Apt. A

Baton Rouge, LA 70802

Dear Ms. Kutlesic,

Thank you for your request for information on the Eating Attitudes Test (EAT). You have permission to use the EAT in your research and clinical work and there is no charge for this permission. I would appreciate you providing me with a copy of any reports or publications in which this instnument is used since it may serve as a useful resource for other researchers and clinicians.

The EDI-2 manual, test booklets, the EDI-2 symptom checklist and profile forms are available from the publisher, Psychological Assessment Resources, P.O. Box 998, Odessa FL 33556.

You can call PAR at 1-800-331-8378.

If you have further questions, please do not hesitate to contact me. Please give donald Williamson my personal regards.

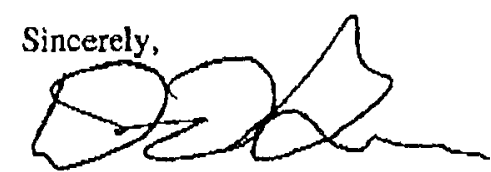

David M. Gamer, Ph.D.
DERARTMEMT OF PJYCHOLOGT

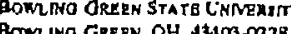

ONNGAL OTREX

7261 W. CENTRAL AVI

TULRDO, OH 43617

TR1 SPHDNE: (419) 843.2300

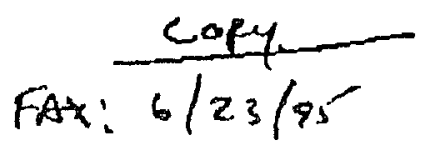




\author{
Vesna Kutlesic, M. A. \\ Louisiana State University \\ Psychology Department. \\ Baton Rouge. LA 70803 \\ (504) 388-8745
}

address correspondence to: 658 N. Bth St. Apt. A

Baton Rouge. LA 70802

(504) 387-6157

May 13, 1994

Mark H. Thelen, Ph.D.

Department of Psychology

210 McAlester Hall

University of Missouri-Columbia

Columbia. Missouri 65211

Dear Dr. Thelen.

My name is Vesna Kutleaic, and I am graduate student at Louisiana State University working under the supervigion of Donald Williamson. Ph.D.. For my doctoral dissertation, I will bo evaluating the psychometric properties of a semi-structured interviow we have doveloped for the diagnosis of the eating disorders. I am writing to request your permigsion for the use and reproduction of the Bulimia Test-Revised. Thank you for your time, and I look forward to your reply.

Ledra

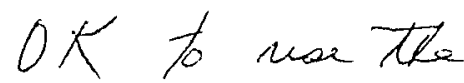

BULIT- P. Pleaer

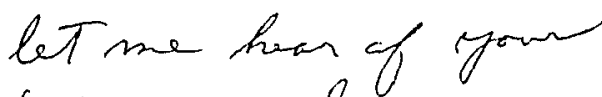

Gridu is . Bive my

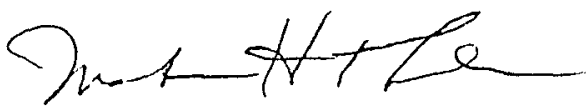

Sincerely,<smiles>C#CC#CCC1CC1CC(C)(C)C</smiles>

Vegna Kutlesic, M.A. 


\section{vita}

Vesna Kutlesic was born January 8, 1968 in Cleveland, Ohio. She attended Kent State University, where she graduated Summa cum Laude with University Honors in December 1988 with a Bachelor of Arts degree in psychology. Vesna earned her Master of Arts degree in psychology from Louisiana State University in May 1992. She is currently a candidate for the Doctor of Philosophy degree in clinical psychology at Louisiana state University, and anticipates graduating in August 1995. 


\section{DOCTORAL EXAMINATION AND DISSERTATION REPORT}

Candidate: Vesna Kutlesic

Major Field: Psychology

Title of Dissertation: The Interview for the Diagnosis of Eating Disorders-IV: Application to DSM-IV Diagnostic Criteria

Approved:

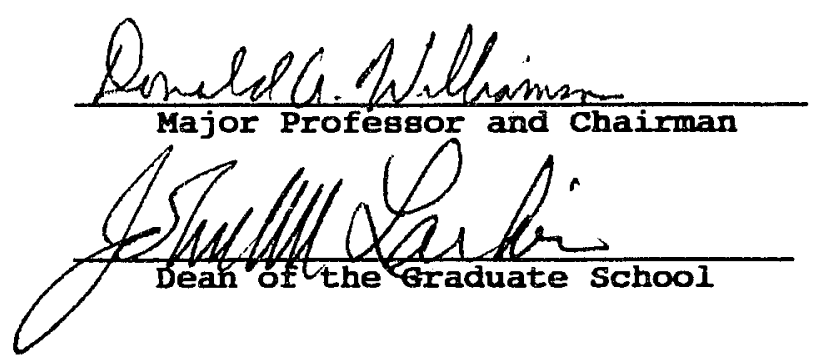

EXAMINING COMMITTEE:

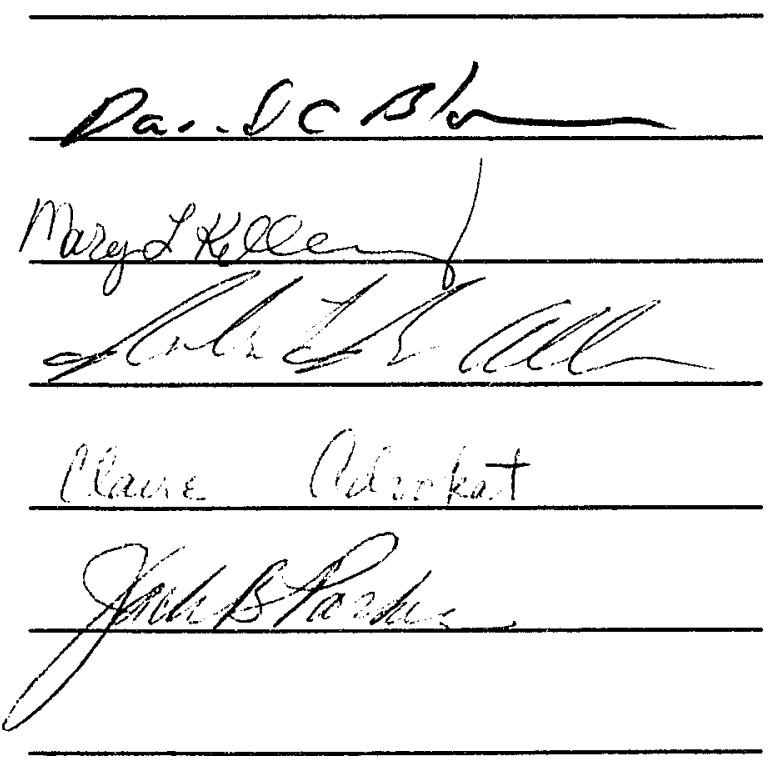

\section{Date of Examination:}

July 10,1995 